\title{
Abstracts of the Eighth Annual Meeting of the Lumbar Spine Research Society \\ Chicago, Illinois • April 9-10, 2015 \\ (DOI: 10.3171/2015.4.FOC-LSRSabstracts)
}

\author{
Paper \# 1. Intraocular Pressure in Patients Undergoing Posterior \\ Lumbar Fusion -- A Prospective, Randomized Trial
}

Sanford E. Emery, MD, MBA; Scott D. Daffner, MD; John C. France, MD; Matthew Ellison, MD; Nina B. Clovis, BS

West Virginia University

Introduction: Ischemic optic neuropathy (ION) resulting in visual loss is a rare but devastating complication in spine surgery. Procedure time, blood loss, prone position and systemic factors may contribute to the risk of perioperative blindness. Elevated intraocular pressure (IOP) results in decreased perfusion and possibly ION. We evaluated the effect of head positioning on IOP in lumbar spine fusion patients.

Methods: Surgeries were performed on 52 patients at one institution. Inclusion criteria were lumbar spine fusion in patients 18-80 years old. Exclusion criteria included eye disease or injury, history of cervical stenosis, neck pain, trauma or current neoplasm. The control group had the head in neutral position with the face parallel to the level operating room table and the experimental group had the neck extended so the face had an angle of inclination of $10^{\circ}$ with the table. All patients were managed with Gardner-Wells tongs and ten pounds of traction on a Jackson table. Using an applanation tonometer, one author made all IOP measurements in pre-operative holding, supine after anesthetic induction, prone after positioning on the table and at regular intervals throughout the case. IOP measurements were recorded with respective time points and corresponding blood pressure and $\mathrm{CO} 2$ values. Independent variables included age, duration of procedure, blood loss, type/amount of fluid replacement, blood pressure, PCO2, gender and head position.

Results: Data were analyzed using ANOVA for categorical risk factors and with regression analysis for continuous risk factors. Mean values for IOP measurements in the prone position were statistically significantly lower in the $10^{\circ}$ elevated group versus the head-neutral group $(\mathrm{p}=0.0014)$. This difference became evident approximately 30 minutes into a case and persisted for the duration. No patient sustained visual loss or any cervical spine related complications.

Conclusion: Ten degree elevation of the head in the prone position for adult lumbar spine fusion patients resulted in statistically significantly lower IOP measurements compared to controls. As lower IOP correlates with increased optic nerve perfusion, this positioning intervention could mitigate the risk of perioperative blindness in spine surgery patients in the prone position.

Paper \# 2. Posterolateral Lumbar Arthrodesis with and without Interbody Arthrodesis for L4-5 Degenerative Spondylolisthesis: A Comparative Value Analysis

Michael B. Gottschalk, MD; Scott Boden, MD; John Heller, MD; S. Tim Yoon, MD PHD; John M. Rhee, MD; Ajay Premkumar, BS; Kyle Sweeney, $M D$

Emory University

Introduction: The addition of an interbody arthrodesis (IBA) to a posterolateral arthrodesis (PLA) has become increasingly popular over the past two decades, yet the potential added value for the patient has not been carefully defined. The authors hypothesized that the addition of IBA to PLA in the setting of L4-5 degenerative spondylolisthesis (DS) will produce added value while incurring minimal additional costs.

Methods: Patients undergoing single level arthrodesis with either PLA alone or PLA+IBA for L4-5 DS from 2004-2012 were identified from our institution's prospective database. Exclusion criteria included multilevel arthrodesis, spinal stenosis requiring decompression at or above L2-3, previous L4-5 spinal fusion, spondylolisthesis of greater than $33 \%$ of the vertebral body, and use of minimally invasive surgery.

Results: 179 patients with an average follow up of 38.7 months met inclusion criteria with 68 having undergone PLA alone and 111 having undergone PLA+IBA. The study was powered to detect smaller differences than the accepted minimal clinically important differences (MCID) for each outcome measure; however, no statistical differences were noted in ODI, SF-36, or fusion rates at 6 months and at over 3 years despite the PLA cohort being significantly older with more medical comorbidities. The addition of IBA did improve lordosis/sagittal balance by 1.5 degrees when compared to the PLA cohort ( $\mathrm{p}=0.04)$. When Costs/QALYs were calculated and normalized for length of stay (LOS), the PLA+IBA cohort demonstrated increased Costs/QALYs at every time point except the extremes of $\operatorname{LOS}(\mathrm{p}=0.01)$.

\begin{tabular}{|c|cc|cc|c|}
\hline \multicolumn{7}{|c|}{ Table 5. Costs/QALYs Normalized by Length of Stay } \\
\hline & \multicolumn{3}{|c|}{ PLA } & \multicolumn{3}{c|}{ PLA+IBA } & \\
\hline Length of Stay (days) & QALYs & Cost/QALYs & QALYs & Cost/QALYs & P.value \\
\hline 2 & 0.74 & $\$ 32,053$ & 0.74 & $\$ 31,791$ & 0.77 \\
\hline 3 & 0.72 & $\$ 35,530$ & 0.71 & $\$ 36,048$ & 0.86 \\
\hline 4 & 0.70 & $\$ 37,819$ & 0.70 & $\$ 39,518$ & 0.59 \\
\hline 5 & 0.70 & $\$ 41,357$ & 0.68 & $\$ 48,954$ & 0.33 \\
6 & 0.66 & $\$ 47,230$ & 0.55 & $\$ 65,301$ & 0.01 \\
7 & 0.67 & $\$ 51,221$ & 0.72 & $\$ 50,577$ & 0.72 \\
\hline
\end{tabular}

Table 5: * denotes that a statistical difference was noted between cost/QALYs. The PLA + IBA incurred higher costs/ QALYs at every time point with the exception of the extremes of LOS.

Discussion/Conclusion: This single center review of open surgical treatment of L4-5 DS demonstrated that the addition of IBA to PLA added cost while not producing superior results in fusion rates, ODI, and SF-36 when compared to PLA alone. On the basis of this data we cannot recommend routine addition of IBA to PLA in patients being treatedsurgically for symptomatic DS.

Paper \# 3. Retracted.

Paper \#4. Effects of Intraoperative Local Steroid Utilization in a Single-Level Minimally Invasive Transforaminal Lumbar Interbody Fusion

Junyoung Ahn, BS; Gabriel Duhancioglu, MS; Rahul Kamath, MS; Ehsan Tabaraee, MD; Daniel D. Bohl, MPH; Khaled Aboushaala, $M D$; Kern Singh, $M D$

Rush University Medical Center

Introduction: Local epidural steroid application may be associated with decreased pain and narcotic utilization in the immediate 
post-operative period following lumbar discectomy. However, local steroid delivery following a lumbar fusion surgery has not been well characterized. As such, the purpose of this study is to characterize the surgical outcomes and narcotic utilization following a 1- level minimally invasive transforaminal lumbar interbody fusion (MISTLIF) as a function of local intra-operative depomedrol utilization.

Methods: A prospective, randomized, single blinded study was performed. Patients were prospectively randomized via a computer number generator into depomedrol (DEPO, 1cc - 40mg) and no depomedrol (NODEPO, 1cc - saline) cohorts. 49 patients undergoing a primary 1-level MIS-TLIF for degenerative spinal pathology were analyzed. Demographics, comorbidity, smoking, visual analogue scale (VAS) scores, Oswestry Disability Index (ODI), Short-form Health Survey (SF-12), operative levels, peri-operative variables, and narcotic utilization were compared between groups. Student's T-test and Pearson's Chi-square analysis were performed for continuous and categorical data, respectively. An alpha level of $<0.05$ denoted statistical significance.

Results: Of the 49 patients included, 29 (59.2\%) received intraoperative depomedrol (1cc-40mg). Demographics, comorbidity burden, smoking status, and operative levels were similar between cohorts. No differences were demonstrated in the pre-operative VAS, ODI, and SF-12. Operative time, estimated blood loss (EBL), and narcotic utilization did not differ between cohorts. Length of hospitalization was greater in the NODEPO patients $(50.5 \pm 21.4$ vs. $41.9 \pm 14.7$ hours; $\mathrm{p}<0.05)$. Post-operative VAS and ODI scores (6- and 12-week) were significantly higher in the DEPO cohort $(\mathrm{p}<0.05)$, but were equivalent at the final 6-month follow-up.

Discussion: The findings of this prospective, randomized blinded

Table 1. Pre- and Post-operative Characteristics for Primary 1-Level MIS TLIF with or without Depomedrol Administration +

\begin{tabular}{cccc}
\hline & Depomedrol & No Depomedrol & p-value \\
Pre-operative SF PCS ${ }^{1}$ & $33.4 \pm 14.4$ & $35.0 \pm 8.7$ & 0.74 \\
Pre-operative SF MCS ${ }^{2}$ & $49.4 \pm 12.1$ & $56.2 \pm 8.9$ & 0.11 \\
Operative Time (min) & $131.0 \pm 27.6$ & $123.2 \pm 21.9$ & 0.32 \\
Estimated Blood Loss (cc) & $61.3 \pm 33.9$ & $57.1 \pm 26.1$ & 0.66 \\
Length of Hospitalization (hours) & $41.9 \pm 14.7$ & $50.6 \pm 21.4$ & $<0.05$ \\
Narcotic Utilization (OME) & & & \\
\hline Oral Morphine Equivalents $\ddagger$ & $114.3 \pm 46.2$ & $109.2 \pm 43.2$ & 0.70 \\
Visual Analogue Scale (VAS) & & & \\
\hline Pre-operative & $5.6 \pm 2.4$ & $4.9 \pm 2.0$ & 0.39 \\
6-week & $5.7 \pm 2.6$ & $2.9 \pm 2.3$ & $<0.05$ \\
12-week & $5.6 \pm 2.5$ & $2.9 \pm 1.7$ & $<0.05$ \\
6-month & $4.8 \pm 2.3$ & $4.6 \pm 2.1$ & 0.91 \\
6-month \& & & & $<0.05$ \\
12-week & $42.8 \pm 21.8$ & $38.3 \pm 15.5$ & 0.52 \\
Pre-operative & $48.7 \pm 22.1$ & $30.9 \pm 9.7$ & $<0.05$ \\
\hline Oswestry Disability Index (ODI) & $47.1 \pm 19.4$ & $24.2 \pm 11.5$ & 0.66
\end{tabular}

† MIS TLIF: Minimally Invasive Transforaminal Lumbar Interbody Fusion $\ddagger$ OME: Sum of OME values from post-operative days 0 and 1

$\$$ Only 8 patients: 5 Depomedrol, 3 No Depomedrol

Only 8 patients: 5 Depomedrol, 3 No Depomedrol
'SF PCS: Short-form health survey (SF-12) Physical Component Summary

'SF PCS: Short-form health survey (SF-12) Physical Component Summary
${ }^{2}$ SF MCS: Short-form health survey (SF-12) Mental Component Summary

${ }^{2}$ SF MCS: Short-form health survey (SF-12)
${ }^{3}$ VAS: Visual Analogue Scale Scores (Back)

${ }^{3}$ VAS: Visual Analogue Scale Sco
${ }^{4}$ ODI: Oswestry Disability Index trial suggest surgical outcomes and narcotic utilization in the immediate post-operative period may be similar between DEPO and NODEPO cohorts. The DEPO patients demonstrated significantly higher post-operative VAS and ODI scores at 6- and 12-weeks.

Conclusions: Patients appear to demonstrate similar post-operative pain levels at final follow up independent of depomedrol administration following a 1-level MIS-TLIF.

Paper \#5. The Local Application of Vancomycin in Spine Surgery Does Not Increase Vancomycin Resistance in Surgical Site Infections

Frank H. Valone, M.D.; Vedat Deviren, MD; Serena S. Hu, MD

University of California, San Francisco

Introduction: Vancomycin placement in the surgical wound has come under recent investigation. Sweet et al. showed a significant reduction in spine surgical site infections. However, no data is available on how this novel treatment has altered bacterial resistance profiles. The aims of this study are to: analyze bacteria and resistance patterns in spine surgery infections, and determine if there is a significant association between Vancomycin placement and Vancomycin resistant bacteria.

Methods: A retrospective consecutive case series analysis was performed on all surgical site infections within an academic tertiary care spine center from 2007 - 2013. Additionally, analysis was performed on 1013 surgical cases completed from 2011-2013 with varying application of Vancomycin to determine association with Vancomycin resistant bacteria. Antibiotic resistance profiles as well as bacteria type were analyzed by epoch. Additionally, association of Vancomycin application and development of Vancomycin resistant bacteria was analyzed by Chi-square analysis.

Results: 126 bacteria were isolated from 81 surgical site infections from 2007-2013. Resistance profiles and bacteria type were analyzed. The most prevalent bacteria isolated: 2007: MSSA (36\%); 2008: MSSA, MRSE (13.5\%); 2009: MRSE (41.2\%); 2010: MRSE (33.3\%); 2011: MSSA (54.6\%); 2012: MSSA (18.8\%); 2013: MSSE (40\%). Only two Vancomycin resistant bacteria were found: Enterococcus Faecium 2008 \& 2012. Additionally, from 2011-2013 Vancomycin was placed 475 times (47\%). The single patient with the Vancomycin resistant bacteria in 2012 did not receive Vancomycin in the surgical wound prior to the development of the infection. There was not a significant association found between Vancomycin application and the development of Vancomycin resistant bacteria $(\mathrm{p}>0.05)$.

Conclusion/Discussion: This study provides retrospective data that suggests that Vancomycin resistant bacteria are rare in spine surgery infections. Additionally, there has not been an increase in Vancomycin resistant surgical site infections. Lastly, there was not a significant association between Vancomycin placed intraoperatively and Vancomycin resistant bacteria. This study is an integral first step in monitoring how the application of Vancomycin will alter the bacteria, and resistance profiles in spine surgical site infections. 
Paper \#6. A Prospective, Multicenter, Randomized Controlled Trial Of Nonsurgical Management Vs. Minimally Invasive Fusion For Sacroiliitis Or Sacroiliac Joint Disruption

David Polly, MD; Daniel Cher, MD; Peter G. Whang, MD, FACS; john glaser, MD; Clay J. Frank, MD; William S. Rosenberg, MD; Scott H. Kitchel, MD; Don A. Kovalsky, MD; Jonathan N. Sembrano, $M D$

\section{University of Minnesota}

Background: Sacroiliac (SI) joint pain is a prevalent but underdiagnosed cause of low back pain. Surgical fusion can relieve pain and improve quality of life in patients who fail nonoperative care. We report a prospective multicenter randomized clinical trial of minimally invasive SI joint fusion using triangular titanium implants or non-surgical management in patients with chronic SI joint pain.

Methods: 148 patients with SI joint dysfunction due degenerative sacroiliitis or SI joint disruption were randomly assigned to either minimally invasive SI joint fusion $(\mathrm{N}=102)$ or non-surgical management (NSM, $n=46$ ). Crossover from NSM to SI joint fusion was allowed only after the 6-month visit was complete. Pain scores and back disability (Oswestry Disability Index) were collected at baseline, and 1,3, and 6 months after surgery or assignment to NSM. Quality of life scores (SF-36 and EQ-5D) were collected at baseline and 6 months. Changes in continuous parameters were assessed with repeated measures analysis of variance. Success rates, (improvement of $>20 \mathrm{~mm}$ on visual analog scale and the absence of severe device-related or neurologic SI joint-related adverse events or surgical revision), were compared using logistic regression.

Results: Mean age was 51 years and $70 \%$ were women. Subjects were highly debilitated at baseline (mean VAS 82, mean ODI 62) and had pain for an average of 6 years. 6 month follow-up was obtained in $>95 \%$. 6 months success was reached in $81 \%$ of the fusion group and $27.5 \%$ in the NSM group $(p<0.0001)$. Mean pain decreased by 42 more points (0-100 scale) in the fusion group vs. the NSM group. Pain relief was greater in the fusion group at all postoperative visits. $48.6 \%$ and $11.4 \%$ of subjects in the fusion and NSM groups had a substantial ( $\geq 15$ point) improvement in ODI. Quality of life improved more in the fusion group and satisfaction rates were high.

Conclusion: Compared to non-surgical management, minimally invasive SI joint fusion using porous, coated triangular titanium implants is more effective in relieving pain, improving function and quality of life in patients with SI joint dysfunction due to degeneration or disruption of the SI joint.

Paper \#7. The Fusion-related And Functional Outcomes Of Open Transforaminal Lumbar Interbody Fusion For Degenerative L/5 Spondylolisthesis

Matthew Colman, MD; Prokopis Annis, MD; Iain Elliott, MD; William R. Spiker, MD; Darrel S. Brodke, MD; Brandon D. Lawrence, $M D$

\section{Midwest Orthopaedics at Rush}

Introduction: The role for addition of interbody fusion to instrumented posterolateral fusion in degenerative spondylolisthesis is unclear. It is not known whether the commonly cited benefit of improved fusion rates applies to this specific population.

Methods: We performed a single center, retrospective casecontrol analysis of 120 consecutive cases of surgically treated grade 1 or 2 L4/L5 degenerative spondylolisthesis. The control group underwent open instrumented posterolateral fusion alone, while the treatment group underwent the addition of open transforaminal lumbar interbody fusion. We measured patient demographics, surgical variables, both preoperative and postoperative spinal alignment parameters, and complication profiles. Primary outcome measures included presence of nonunion based on $\mathrm{CT}$, radiographic fusion grade according to the Bridwell criteria, and patient-reported health related quality of life metrics including the ODI, EQ5D, and 10-point VAS back pain scale.

Results: There were 120 patients, 55 in the control group and 65 in the treatment group, with a mean follow-up of 30.5 months. In univariate analysis, the interbody and non-interbody groups had similar $(\mathrm{p}>0.05)$ rates of CT-based nonunion $(7.7 \%$ vs. $9.1 \%)$, mean Bridwell grade (1.3 vs. 1.4), and final ODI (27.3 vs. 24.7), EQ5D (0.61 vs. 0.69$)$, and VAS back pain (3.2 vs. 1.9) scores. In additional analysis of the effect of all other covariates on fusion outcome, only active smoking trended at a higher rate in the nonunion population $(20 \%$ vs. $5.5 \%, p=0.08)$. There was no statistically significant effect of pelvic incidence, sagittal vertical axis, or lumbar lordosis on fusion rate. In multivariable modeling of treatment effect, use of an interbody cage had no statistically significant impact on the incidence of CT-based nonunion, final follow-up Bridwell grade, or final follow-up ODI, EQ5D, or VAS back pain scores.

Conclusions: For degenerative spondylolisthesis at L4-L5, nonunion rates are low, and addition of an interbody fusion procedure does not appear to offer significant benefit with regard to fusion rates or patient-reported health-related quality of life outcomes.

Discussion: Continued refinements to the indications for lumbar interbody fusion are required. These devices may be more appropriate for clinical settings outside degenerative grade 1 or 2 L4/5 spondylolisthesis.

\section{Paper \#8. The Rate Of Lumbar Spinal Fusion Following Microdiscectomy}

Steven McAnany, MD; Samuel Overley, MD; Samuel Cho, MD; Andrew Hecht, MD; Sheeraz Qureshi, MD, MBA; Holt S. Cutler, BSE; William J. Rubenstein, B.A.; Matthew Anderson, BS

\section{Mount Sinai Medical System}

Introduction: Lumbar microdiscectomy is a highly utilized procedure for patients with a symptomatic herniated nucleus pulposus (HNP) recalcitrant to conservative measures. This procedure has a successful track record and has been validated throughout the literature. The rate of recurrence after a single level lumbar microdiscectomy ranges from $5-11 \%$, with revision microdiscectomy yielding similar success rates the index procedure. There are no studies in the literature that report in the rate of subsequent fusion following lumbar microdiscectomy with either an open or MIS approach.

Methods: A consecutive series of 885 patients with symptomatic single-level lumbar HNPs underwent microdiscectomy via a miniopen approach (499) or with an MIS dilator tube (386). Only revision procedures performed at the index level and involving no other levels were included in the revision data subsets. The indication for revision microdiscectomy was symptomatic reherniation that had failed appropriate conservative management. Fusion was indicated after a failed revision microdiscectomy or evidence of interval spondylosis, listhesis, or instability following index microdiscectomy. Normative data values respective to overall rates of revision procedures were analyzed with a chi-squared test.

Results: Of the 885 total lumbar microdiscectomies performed over an 11-year period, 499 procedures utilized a mini-open approach and 386 with an MIS approach. Overall, 5.8\% (51/885) of patients underwent a revision microdiscectomy at the index level. The average time to average revision discectomy was 25.6 months. The rate of fusion following lumbar microdiscectomy was found to be $1.8 \%(16 / 885)$. The average time to fusion following discectomy was 20.1 months. Comparing the rates of fusion following open or MIS discectomy were not found to be statistically significant $(\mathrm{p}=0.62)$.

Conclusions: This is the first study to assess the rate of subse- 
quent fusion following lumbar microdiscectomy with a mini-open or MIS approach. Our results indicate that there is a low rate of fusion $(1.8 \%)$ following lumbar microdiscectomy. Furthermore, there were no differences found in the rates of fusion between a mini-open or MIS approach. Our rates of revision discectomy at the index level $(5.8 \%)$ are consistent with previously published results, and further validate the findings of this study. Additional information may be gathered from a sub-group analysis to identify possible predictors of fusion following lumbar microdiscectomy.

\section{Paper \#9. Hip Flexion Weakness After Xlif}

Jim A. Youssef, MD; Robert E. Isaacs, MD; Juan S. Uribe, MD

\section{Durango Orthopedics}

Background: Access to the disc space in the lateral approach to the lumbar spine is gained by traversing the lumbar plexus and psoas muscle. In some patients this muscle retraction results in pain that manifests as hip flexion weakness (HFW). The purpose of this analysis is to evaluate the relationship between intraoperative metrics and the presence of post-operative HFW.

Methods: As part of a study on EMG monitoring throughout the XLIF procedure, patients from 21 sites who underwent XLIF at L4-5 with or without treatment at additional levels were enrolled in a prospective, nonrandomized, multi-center, institutional review board (IRB)-approved, clinical study. Data collection included demographics, pre-and postoperative 0-5 motor function and intraoperative collection of total number of interbody levels fused, total procedure time, estimated blood loss, and use of bone morphogenetic protein (BMP). Results from patients with postoperative hip flexion-weakness were compared to those without.

Results: Three hundred twenty three (323) patients were enrolled. Postoperatively 167 had no decrease in motor or sensory function, 91 had HFW with or without additional motor/sensory decreases in function. The remaining 65 patients were removed from analysis due to either missing data or the presence of postoperative decreases in motor/sensory function without HFW. Women vs. men were more likely to develop HFW (45\% of females vs. $18 \%$ of males, $\mathrm{p}<0.001)$, as were smokers vs non-smokers $(51 \%$ of smokers vs. $33 \%$ of non-smokers). Factors that were not significantly different between patients with and without HFW were body mass index $(\mathrm{p}=0.301)$, age $(\mathrm{p}=0.871)$, preoperative diagnosis of scoliosis $(\mathrm{p}=0.0711)$, number of levels fused $(\mathrm{p}=0.095)$, intraoperative use of BMP $(p=0.221)$, total procedure time $(p=0.292)$, and estimated blood loss $(\mathrm{p}=1.00)$.

Conclusions: In this population, HFW was greater in women and patients who smoked. On average, patients who developed postoperative HFW were treated at more levels, though this measure was not significantly different.

\section{*Paper \# 10. Prospective Evaluation of Radiculitis Following BMP-2 use for Interbody Arthrodesis in Spine Surgery}

Arjun S. Sebastian, MD; Paul M. Huddleston, MD; Mark A. Pichelmann, MD; Bradford L. Currier, MD; Vickie M. Treder, B.A.; Ahmad Nassr, MD

\section{Mayo Clinic}

Introduction: Concerns have been raised regarding the use of BMP in transforminal interbody fusion (TLIF). BMP use close to nerve roots may cause inflammation or heterotopic ossification leading to postoperative radiculitis. Prospective studies regarding use of BMP in TLIF are lacking. The purpose of this study is to evaluate the safety and efficacy of BMP-2 use in TLIF with regards to postoperative radiculitis.
Methods: Between May 2009 and September 2013, 77 patients were enrolled in this prospective study. Use of BMP-2 was determined on an individual basis. Demographic and operative characteristics were recorded. Fusion rates were determined by a blinded reviewer and several functional outcomes were collected including visual analog pain scores (VAS), Oswestry Disability Index (ODI), Sciatica Bothersome Index (SBI), and Short Form-36 (SF-36) scores. Outcomes data was collected at each follow up visit.

Results: Of the 77 patients enrolled, 29 received BMP and 48 did not. Increased use of iliac crest autograft $(41.7 \%$ vs $6.9 \%, p=$ $.001)$ and blood loss (1035.2 vs $608.6 \mathrm{cc}, \mathrm{p}=.042)$ were noted in the control group. There were significant improvements in postoperative leg pain as measured by VAS leg and SBI scores for the entire cohort without clinically significant differences between the BMP and control groups. Significant improvements were also found in VAS back, ODI, and SF-36 scores. A significantly increased 6 month fusion rate was noted in the BMP group $(82.8 \%$ vs $55.3 \%$, p $=.024)$ with no significant difference at 12 months and 24 months followup. Heterotopic ossification was appreciated in 7 patients, 6 in the BMP group, without any clinical impact. Two reoperations occurred in each group for misplaced hardware, hematoma evacuation, and revision decompression.

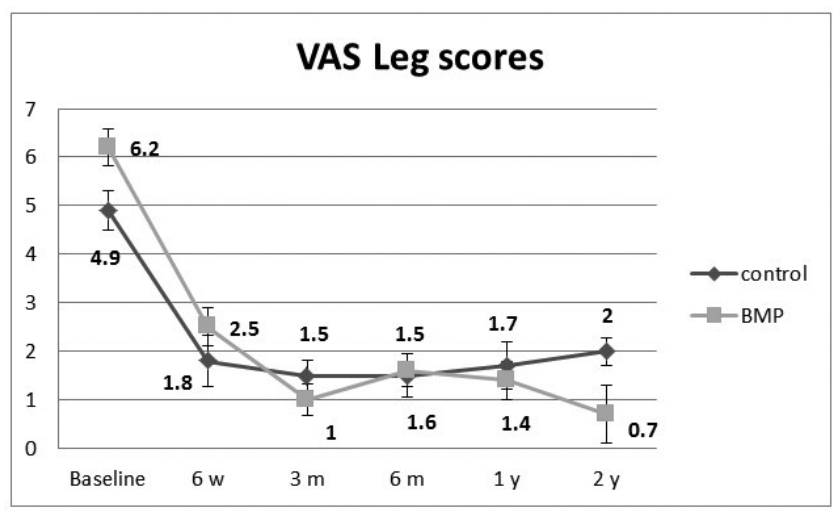

Conclusions: In this prospective trial, the use of BMP in TLIF did not lead to significant postoperative radiculitis as measured by VAS leg and SBI scores. BMP did increase short term fusion rates and led to less blood loss. Back pain and other functional outcome scores also improved following TLIF with no difference between BMP and control groups. Careful use of BMP in TLIF appears to be both safe and efficacious.

* Off Label Usage - 2. The FDA has not cleared the following pharmaceuticals and/or medical devices for the use described in this presentation. Please explain below.

This study examines the use of BMP in TLIF. This is currently an off-label indication for BMP use.

\section{Paper \#11. Risks Factors for Blood Transfusion with Primary Posterior Lumbar Fusion}

Bryce A. Basques, BS; Andre M. Samuel, BBA; Adam M. Lukasiewicz, MSc; Matthew L. Webb, AB; Daniel D. Bohl, MPH; Nicholas Golinvaux, BA; Jonathan Newman Grauer, MD

\section{Yale School of Medicine}

Introduction: Blood transfusion is a relatively common occurrence for patients undergoing primary posterior lumbar fusion. There is limited information available describing which patients are at increased risk for blood transfusion. In addition, the relationship 
Table 1. Associations with blood transfusion during or after primary lumbar fusion.

\begin{tabular}{lcr}
\hline \multicolumn{1}{c}{ Variable } & Relative Risk & P-value \\
\hline Patient/operative factors associated with blood transfusion & & \\
Age 60-69 vs Age $<50$ & 1.7 & $<0.001$ \\
Age $\geq 70$ vs Age $<50$ & 1.8 & $<0.001$ \\
ASA class $\geq 3$ & 1.4 & 0.001 \\
Two levels vs one level & 2.0 & $<0.001$ \\
Three or more levels vs one level & 3.3 & $<0.001$ \\
Operative time $\geq 310$ minutes & 4.0 & $<0.001$ \\
& & \\
Postoperative outcomes associated with blood transfusion & & \\
Readmission within 30 days & 1.6 & 0.003 \\
Any complication & 2.2 & $<0.001$ \\
Postoperative ventilator $>$ 48 hours & 5.5 & 0.002 \\
Reintubation & 3.3 & 0.007 \\
Cardiac arrest & 5.2 & 0.033 \\
Myocardial infarction & 3.3 & 0.043 \\
Sepsis & 2.5 & 0.012 \\
Return to operating room & 1.9 & 0.001 \\
Deep surgical site infection & 2.8 & 0.019 \\
Pneumonia & 4.7 & $<0.001$ \\
Pulmonary embolism & 4.7 & 0.001 \\
Deep vein thrombosis & 2.9 & 0.008 \\
\hline
\end{tabular}

between blood transfusion and short-term postoperative outcomes is poorly characterized.

Methods: The prospectively collected American College of Surgeons National Surgical Quality Improvement Program (ACSNSQIP) database was used to identify patients undergoing primary posterior lumbar fusion from 2011 to 2013. Patient characteristics were tested for association with the occurrence of a blood transfusion from the beginning of the procedure up to 30 days postoperatively using multivariate Poisson regression with robust error variance. In addition, the association between blood transfusion and postoperative complications was tested using multivariate analysis. The association between blood transfusion and postoperative length of stay was assessed using multivariate linear regression.

Results: Of the 4,223 primary posterior lumbar fusion patients that met inclusion criteria, 704 patients $(16.7 \%)$ had a blood transfusion. Multivariate analysis found that age $\geq 60$, ASA class $\geq 3$, two or more levels, and operative time $\geq 310$ minutes were associated with blood transfusion (Table 1). A blood transfusion significantly increased the risk of readmission within 30 days, as well as the risk of multiple complications including prolonged postoperative ventilator use, reintubation, cardiac arrest, myocardial infarction, sepsis, return to the operating room, deep surgical site infection, pneumonia, pulmonary embolism, and deep vein thrombosis. Blood transfusion was also associated with an increase in postoperative length of stay of 1.6 days ( $\mathrm{p}<0.001)$.

Conclusions: One in six patients receive a blood transfusion while undergoing pri-

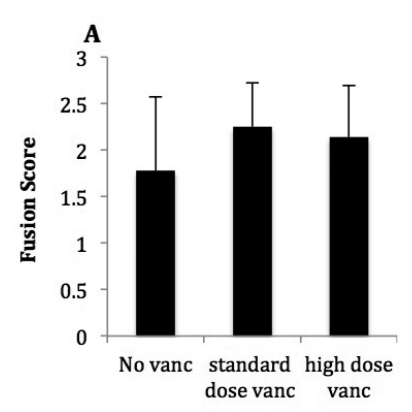

Figure 1: vancomycin: $p=0.53$ ) mary posterior lumbar fusion. Patients with increased age, ASA class, number of levels, and operative time were found to have an increased risk of blood transfusion. Even after controlling for preoperative factors associated with transfusion, receiving a blood transfusion significantly increased the risk of multiple complications including infections, thromboembolic events, readmission, as well as increased average length of stay by a day and a half. By identifying lumbar fusion patients at increased risk of blood transfusion, strategies to minimize effective blood loss might be considered in patients with such factors, such as cell saver or antifibrinolytics.

Paper \# 12. The Effect of Vancomycin Powder on Bone Healing in a Rat Spinal Arthrodesis Model

Marco C. Mendoza, MD; Kevin A. Sonn, $M D$; Sharath S. Bellary, MD; Abhishek S. Kannan, BS; Sean M. Mitchell, BS; Gurmit Singh, BS; Christian Park, B.S.; Chawon Yun, Ph.D.; Stuart R. Stock, PhD; Erin L. Hsu, PhD; Wellington K. Hsu, MD

\section{Northwestern University}

Introduction: Surgical site infections (SSIs) after spinal surgery occur in $1-10 \%$ of patients. Such complications are devastating to patients and the healthcare system. As Staphylococcus aureus is the most common organism responsible for SSIs, vancomycin powder has the potential to serve as a simple, cost-effective solution to the problem. Although in vitro studies suggest that vancomycin is cytotoxic to differentiating osteoblasts, the effect of vancomycin powder application on the rates of spinal arthrodesis has not been properly evaluated. This study aims to quantify the impact of vancomycin powder application on new bone formation and spine fusion rates in a rat posterolateral arthrodesis model.

Methods: Thirty-six female Sprague-Dawley rats underwent a posterolateral lumbar spinal fusion at the L4 and L5 vertebrae.
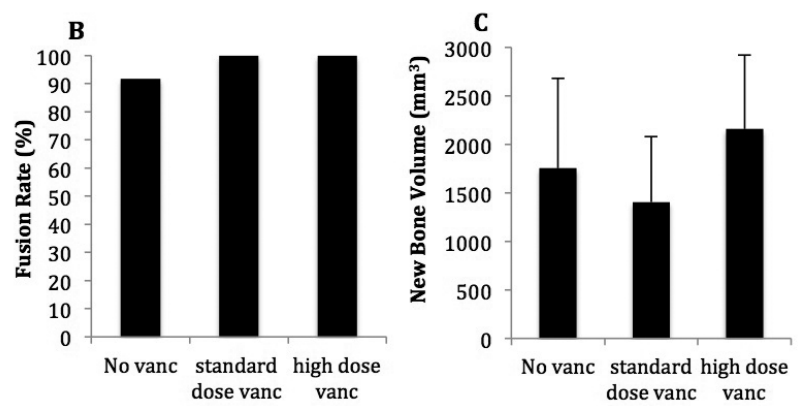

(A) Fusion scores from blind manual palpation analysis at 8 weeks post-operation. (B) Fusion rates of each treatment-based manual palpation scores, where an average score greater than or equal to 1.0 was considered solidly fused. No significant difference was found in fusion scores among the standard dose, high dose vancomycin and control groups. (C) The successfully fused specimens were analyzed by microcomputed tomography $(\mu \mathrm{CT})$ to compare fusion mass volume $\left(\mathrm{mm}^{3}\right)$. There were statistically no significant differences between the vancomycin-treated groups relative to no-vancomycin controls (control vs. standard dose vancomycin: $p=0.57$; control vs. high dose 
Fusion was elicited via implantation of an absorbable collagen sponge containing $3 \mu \mathrm{g}$ rhBMP-2. Rats were divided into three groups: no vancomycin (control), standard dose vancomycin, and high dose vancomycin, which was applied to the fusion bed. Spines were harvested and evaluated at 8 weeks post-operatively using radiographs, fusion scoring, microCT, and histologic analysis.

Results: Qualitative radiographs demonstrated equivalent bridging bone formation in all groups. No significant differences in fusion scores were seen in the standard-dose (2.25) or high-dose (2.13) treatment groups relative to untreated control animals (1.78; Figure 1A). Similarly, fusion rates were not significantly different between vancomycin-treated animals (100\% for both groups) and control animals (92\%; Figure 1B). Quantification of new bone formation via microCT imaging revealed no significant differences in the volume of newly regenerated bone among groups (Figure 1C).

Discussion/Conclusion: This is the first in vivo study to specifically address pseudarthrosis with topically-applied vancomycin. Our results demonstrate that vancomycin powder does not inhibit fusion rates at an equivalent wt $\%$ dose to what is routinely used by surgeons. Moreover, bone formation and fusion rates were not reduced even after administration of a vancomycin dose that is ten-fold higher than that which is typically administered clinically. Our findings suggest that if there is a critical threshold above which vancomycin inhibits bone healing, such a dose is out of the range which might be considered reasonable for clinical use.

Paper \# 13. Local Tumor Necrosis Factor- $\alpha$ (TNF- $\alpha$ ) Decreases Fusion Rates in a Rat Mode

John Koerner, MD; Dessislava Z. Markova, Ph.D.; Gregory D. Schroeder, MD; Alexander R. Vaccaro, MD, PhD; David Greg Anderson, MD; Christopher K. Kepler, MD, MBA

The Rothman Institute at Thomas Jefferson University

Introduction: The local inflammatory response that occurs during the process of spinal fusion involves cytokine and growth factor expression. One inflammatory cytokine involved in this process, TNF- $\alpha$, has demonstrated various effects on bone formation. However, the optimal local environment for spinal fusion, including the levels of TNF- $\alpha$ has not been defined. The purpose of this study is to determine the effects of local TNF- $\alpha$ during spinal fusion in a rat model.

Methods: Thirty male Wistar Kyoto rats underwent L4-5 postero-

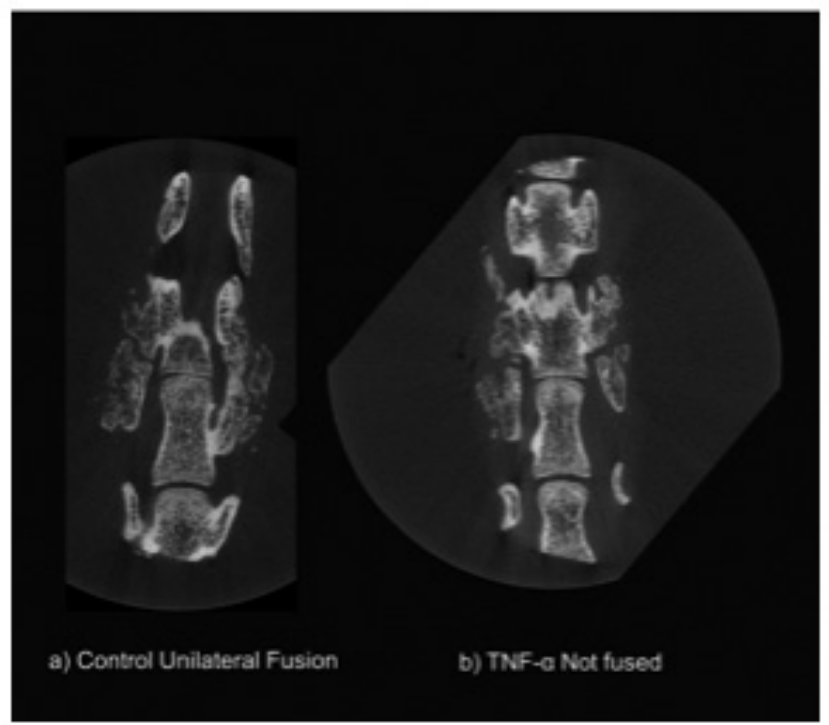

lateral intertransverse lumbar fusion with the addition of demineralized bone matrix (DBM). After decortication with a high speed burr, approximately $0.4 \mathrm{~cm} 3$ of DBM was added to each fusion site. A low dose of TNF- $\alpha(20 \mu \mathrm{L}$ of $50 \mathrm{ng} / \mathrm{mL})$ on an absorbable collagen sponge was added to the fusion site of the treatment groups. Animals sacrificed at day 2 and day 4 had the local fusion mass harvested and processed for cytokine analysis with ELISA. Serum was also collected by cardiac puncture. Animals sacrificed at 4 weeks were assessed by manual palpation and microCT for fusion, as well as serum cytokine levels with ELISA.

Results: The addition of TNF- $\alpha$ at the time of surgery created significantly higher levels of TNF- $\alpha$ in the fusion mass at day 2 $(\mathrm{p}=0.026)$, but not at day $4(\mathrm{p}=0.143)$ compared to controls. There were no differences in the levels of Interleukin-1 $\beta$ (IL-1 $\beta$ ) at the fusion site at day $2(\mathrm{p}=0.917)$ and day $4(\mathrm{p}=0.154)$ in animals treated with local TNF- $\alpha$ versus controls. There was a trend towards increased systemic serum TNF- $\alpha$ levels at 4 weeks in the treatment group $(\mathrm{p}=0.071)$, but no differences at day 2 or day $4(\mathrm{p}=0.572$, $\mathrm{p}=0.728$ respectively) At four weeks, $4 / 5$ of the controls had fused based on manual palpation, compared to $0 / 5$ in the TNF- $\alpha$ group $(p=0.048)$. Based on MicroCT, $2 / 5$ of the controls were fused bilaterally, $2 / 5$ unilaterally, and one was not fused. For the TNF- $\alpha$ group, $1 / 5$ fused unilaterally, and 4/5 were not fused $(\mathrm{p}=0.071)$ (Figure 1 ).

Conclusion: The local addition of TNF- $\alpha$ to the fusion site decreases the rate of successful fusion in a rat model based on manual palpation and MicroCT. The expression of this pro-inflammatory cytokine may vary in patients undergoing spine fusion, and over expression may influence the chance for successful fusion.

Paper \# 14. In Vivo Annular Repair using High-Density Collagen Gel Seeded with Fibrochondrocytes

Yu Moriguchi, MD, PhD; Brandon Borde, BS; Thamina Khair, BA; Marjan Alimi, MD; Lawrence Bonassar, PhD; Roger Härtl, MD

\section{Weill Cornell Medical College}

Introduction: Discectomy of herniated intervertebral discs (IVDs) successfully alleviates associated neurological symptoms but fails to repair the underlying degenerative disc process. Persistent annular defect post discectomy is associated with increased risk of reherniation, progressive IVD degeneration, and chronic low back pain. Recently, we demonstrated the ability of riboflavin crosslinked high-density collagen gels (HDC) to facilitate annular repair in vivo [Grunert P, Spine 2014]. In this study, AF fibrochondrocytes were seeded in the HDC gel to enhance the reparative process at the site of annular defect.

Methods: 24 athymic rats, punctured with an 18-gauge needle in the tail disc, were divided into 3 groups: 1) untreated $(n=4) ; 2)$ injected with crosslinked HDC $(\mathrm{n}=10) ; 3)$ injected with fibrochondrocyte-laden crosslinked HDC $(n=10)$. Sheep AF fibrochondrocytes labeled with DiI at a concentration of a million cells $/ \mathrm{ml}$ were mixed with HDC gels prior to injection. At 1,2, and 5-week time points, a series of in vivo $\mathrm{X}$ ray and $7 \mathrm{~T}$ MRI were performed to assess disc height index of treated segments and nucleus pulposus (NP) size and hydration based on a preexisting method developed by our group using T2-relaxation times [Grunert P, Spine 2014]. Histological assessments were performed to evaluate the viability of implanted cells and the degree of annular repair.

Results: Compared to the untreated puncture group, at 1 week, both HDC gel groups showed significantly higher retention of NP size and hydration while only the cell-laden group better maintained NP retention and disc height up to 5 weeks. The untreated discs showed substantial NP herniation by 2 weeks and NP absence with signs of degeneration by 5 weeks (Fig 1a, b). Histological assessments indicate that while gels influence the sealing of the defect, the addition of cells leads to more abundant tissue growth and extracellular matrix at the site of annular defect, accelerating the reparative 
A

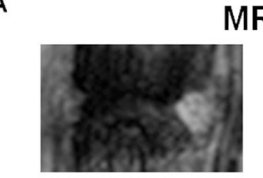

Untreated Control
MRIs at 2 weeks

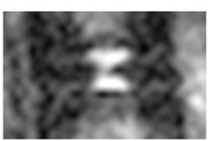

Acellular Gel

NP size

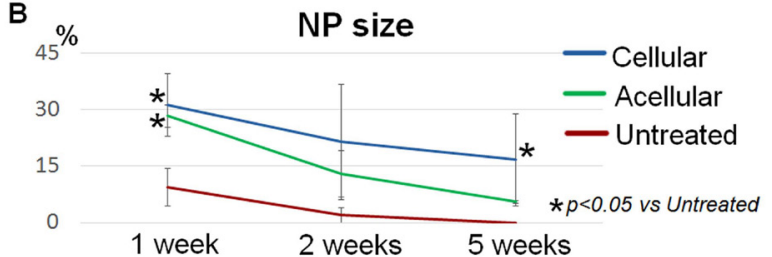

C

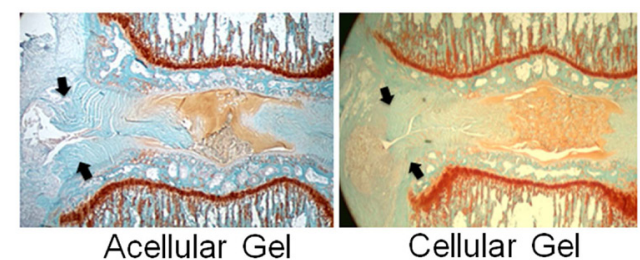

Arrows denote reparative tissue at the site of annular defect.

D

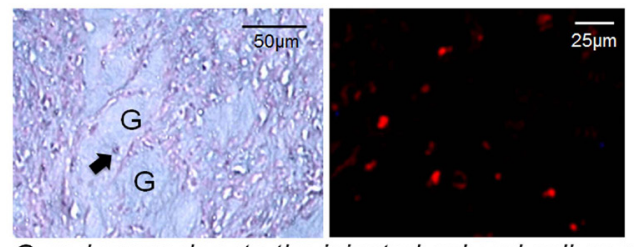

$G$ and arrow denote the injected gel and cell, respectively.

process as early as 2 weeks (Fig 1d). DiI detected implanted fibrochondrocytes in the process of reorganization within gels (Fig 1d).

Conclusions: Our results demonstrate that AF fibrochondrocyteladen HDC gels can improve the annular repair, thereby suggesting its potential feasibility in cell-based therapy for AF.

Paper \# 15. The Effects of Substance P Administration on the Intervertebral Disc in a Rat Organ Culture Model

John Koerner, MD; Dessislava Z. Markova, Ph.D.; Gregory D. Schroeder, MD; Alexander R. Vaccaro, MD, PhD; David Greg Anderson, MD; Christopher K. Kepler, MD, MBA

The Rothman Institute at Thomas Jefferson University

Introduction: In the intervertebral disc (IVD), substance P (SP), a nociceptive neurotransmitter, has been used as a specific marker to identify ingrowth of nerves, which may transmit painful stimuli in degenerative discs. The addition of SP to human IVD cells has also been demonstrated to upregulate inflammatory mediators in a cell culture model. Previous models investigating disc degeneration, such as the needle puncture model, cause physical disruption of the disc, which does not replicate degenerative disc disease. The rat organ culture model allows for analysis of the disc/endplate unit without disruption. The effects of SP on the IVD are unknown in this model.

Methods: Six adult Wistar rats were sacrificed and lumbar discs $(n=6 /$ animal) were harvested. The endplates were dissected and maintained in organ culture media with antibiotics for 8 days. Experimental discs were cultured in media containing antibiotics and Substance $\mathrm{P}(100 \mu \mathrm{M})$. At the time of harvest, the 6 lumbar discs from each animal were pooled together to create one sample for annulus fibrosus (AF) and nucleus pulposus (NP) tissue. The

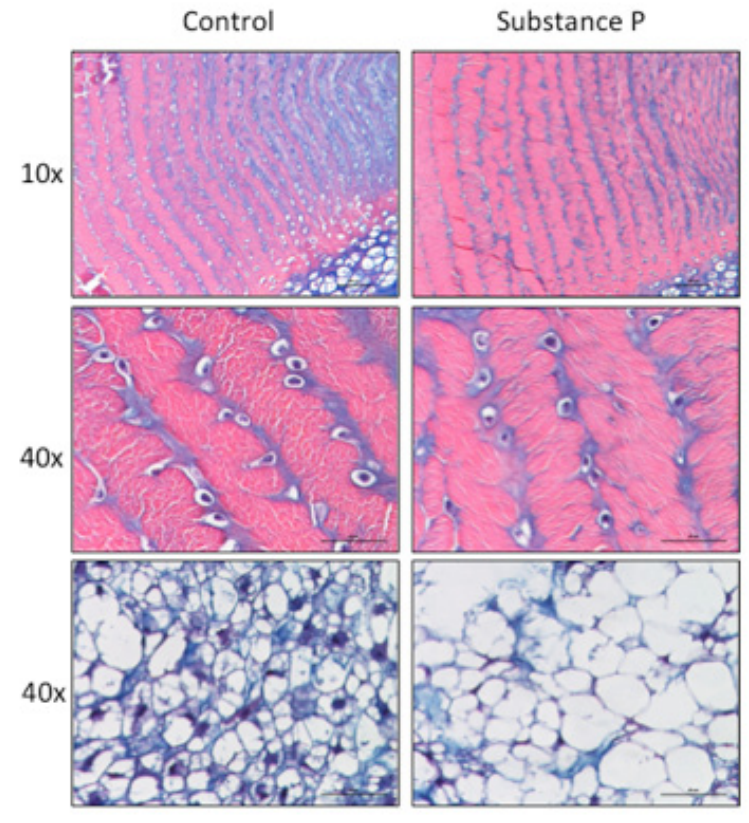

conditioned medium from the organ culture was probed for cytokine production using a rat cytokine antibody array and chemiluminescence was detected. Real-time RT-PCR analysis was completed for confirmation of cytokine expression from NP tissue.

Results: After treatment with SP, the conditioned medium demonstrated an increase in the production of pro-inflammatory cytokines and growth factors (Interleukin-6 (IL-6), CXCL5, vascular endothelial growth factor (VEGF)) compared to controls $(\mathrm{p}=0.09$, $0.04,0.03$, respectively). There was decreased production of Agrin, CD86, and Fractalkine after treatment with SP compared to controls ( $\mathrm{p}=0.006,0.049,0.019$, respectively). On real-time RT-PCR, the NP tissue demonstrated increased expression of IL-6 and matrix metalloproteinase-3 (MMP-3) compared to controls ( $\mathrm{p}=0.001$, 0.059 respectively). Cellular hypertrophy and matrix degradation of nucleus pulposus (NP) tissue and lower cell numbers were seen in AF tissue in discs treated with SP (Figure 1).

Conclusion: Treatment of the IVD with Substance P leads to upregulation of inflammatory cytokines and growth factors, decreased expression of proteoglycan, and created phenotypic changes of degeneration in an organ culture model. These findings demonstrate new evidence that Substance P may be an important regulator of disc degeneration.

Paper \# 16.Predicting Postoperative Morbidity and Readmission for Revision Posterior Lumbar Fusion

Bryce A. Basques, BS; Matthew L. Webb, AB; Andre M. Samuel, BBA; Adam M. Lukasiewicz, MSc; Daniel D. Bohl, MPH; Nicholas Golinvaux, BA; Jonathan Newman Grauer, MD

Yale School of Medicine

Introduction: The need for revision of a lumbar fusion is an unfortunate occurrence that has been previously shown to carry increased risks to patients. However, there is little known about specific risk factors for complications and readmission following revision lumbar fusion. The purpose of this study is to use a large, national sample to identify patient and operative factors that are associated with development of these adverse outcomes.

Methods: The prospectively collected American College of Surgeons National Surgical Quality Improvement Program (ACS- 
Table 1. Significant results of multivariate analyses for the association of risk factors with any adverse event, severe adverse events, and readmission within 30 days.

\begin{tabular}{lcc}
\hline \multicolumn{1}{c}{ Outcome/Risk Factor } & RR & P-value \\
\hline Any adverse event & & \\
Pulmonary disease & 1.8 & 0.034 \\
Two levels vs one level & 1.8 & 0.049 \\
Three or more levels vs one level & 2.3 & 0.025 \\
Operative time $\geq 300$ minutes & 2.2 & 0.002 \\
& & \\
Severe adverse events & & \\
Two levels vs one level & 2.4 & 0.027 \\
Three or more levels vs one level & 3.0 & 0.042 \\
Operative time $\geq 300$ minutes & 2.4 & 0.013 \\
& & \\
Readmission & & \\
Two levels vs one level & 3.2 & 0.005 \\
Three or more levels vs one level & 4.0 & 0.014 \\
BMI 25-30 vs BMI $<25$ & 10.2 & 0.019 \\
BMI 30-35 vs BMI $<25$ & 11.9 & 0.015 \\
BMI $\geq 35$ vs BMI $<25$ & 8.4 & 0.039 \\
\hline
\end{tabular}

$\mathrm{BMI}=$ body mass index, $\mathrm{RR}=$ relative risk.

NSQIP) database was used to identify patients undergoing revision posterior lumbar fusion from 2005 to 2013. Patient characteristics were tested for association with any adverse event, severe adverse events, and readmission within 30 days postoperatively using multivariate Poisson regression with robust error variance.

Results: A total of 763 revision posterior lumbar fusion patients were identified. Of these, $62(8.1 \%)$ had an adverse event and 38 $(5.0 \%)$ had a severe adverse event. Of the 688 patients that had readmission data (90.2\% of all patients), 36 were readmitted within 30 days $(5.2 \%)$. Any adverse event was associated with pulmonary disease, two or more levels, and operative time $\geq 300$ minutes on multivariate analysis (Table 1). Severe adverse events were associated with two or more levels and operative time $\geq 300$ minutes. Readmission was associated with two or more levels and BMI above $25 \mathrm{~kg} / \mathrm{m} 2$.

Conclusions: Short-term postoperative complications are common after revision posterior lumbar fusion, with a rate of $8.1 \%$ in this national sample. Approximately five percent of patients are readmitted within 30 days of the procedure. In general, longer, more extensive procedures were associated with increased risk of postoperative complications and readmission. Importantly, obesity was found to be a significant risk factor for readmission in these patients, and this factor may be modifiable in the preoperative period.

Paper \#17. Cost and Quality of Life Implications of Adjacent Segment Disease Following Lumbar Fusion

Vincent J. Alentado, BS; Daniel Lubelski, BS; Andrew T. Healy, MD; Jacob A. Miller, BS; Michael P. Steinmetz, MD; Ed C. Benzel, MD; Thomas E. Mroz, MD

\section{Cleveland Clinic}

Introduction: Revision surgery for adjacent segment disease (ASD) has been shown to improve quality of life (QOL) in a cost- effective manner. However, the QOL and financial implications of developing ASD have not been well described in the literature.

Methods: Individuals with ASD were matched to control patients without ASD that underwent the same surgery, with the same length of fusion, and were of similar age, and body mass index. Demographic, operative, postoperative, QOL, and cost data were collected for both the ASD and control groups, up to 1-year followup.

Results: We reviewed 137 patients that underwent primary lumbar fusion, and identified $13(9 \%)$ who developed ASD. The average follow-up for the ASD group was 53.8 months and for the control group was 45.1 months. ASD was defined as radiographic and clinical findings requiring corrective surgery for pathology adjacent to the original fusion. No significant differences in demographic or operative characteristics were observed between the ASD and the matched control group. In the ASD group, the average preoperative quality adjusted life years, pain disability questionnaire scores, and patient health questionnaire- 9 scores were $0.576 \pm 0.284$, $94.2 \pm 34.6$, and $12.0 \pm 8.8$, respectively. In the control group, the average preoperative QALYs, PDQ scores, and PHQ-9 scores were $0.629 \pm 0.200,79.7 \pm 23.9$, and $5.9 \pm 5.5$, respectively. The ASD group had significantly worse EQ-5D Health State scores 6 months following both primary fusion and revision operations, compared to controls who did not develop ASD ( $\mathrm{p}=0.03$ and $\mathrm{p}=0.05$, respectively). Furthermore, the ASD group had worse QALYs 1 year after primary fusion and revision operation compared to controls (differences of $0.311, \mathrm{p}=0.002$ and $0.254, \mathrm{p}=0.02$, respectively). Patients with ASD also had worse PHQ-9 scores at 6 months following revision operation compared to primary fusion $(\mathrm{p}=0.003)$. With respect to financial costs, the ASD group had significantly higher costs related to hospital admission $(\$ 12,130 \pm 5435$ vs. $\$ 5,579 \pm 2781, \mathrm{p}<0.0001)$, surgery $(\$ 9,534 \pm 3515$ vs. $\$ 4,240 \pm 1187, \mathrm{p}<0.0001)$, imaging $(\$ 712 \pm 884$ vs. $\$ 205 \pm 211, \mathrm{p}=0.02)$, and total direct costs $(\$ 26,774 \pm 11165$ vs. $\$ 13,861 \pm 5111, \mathrm{p}<0.0001)$ when compared to the control group. There were no significant differences in indirect costs between the two groups.

Conclusions: This study is the first quantification of the impact of ASD on patients' quality of life and associated costs for care. We found that ASD was associated with lower quality of life outcomes and a significantly greater overall cost as compared to patients undergoing primary lumbar fusion.

Paper \# 18. Of 5,267 Lumbar Posterior Fusions, 4.35\% Of Patients Readmitted Within 30 Days: Surgical Site Issues The Most Common Reasons For Readmission

Matthew L. Webb, AB; Andre M. Samuel, BBA; Adam M. Lukasiewicz, MSc; Bryce A. Basques, BS; Daniel D. Bohl, MPH; Arya G. Varthi, M.D.; Jonathan Newman Grauer, $M D$

\section{Yale School of Medicine}

Background: The Affordable Care Act codifies penalties for hospital readmissions. Large clinical outcomes databases can be used to quantify postoperative complications and readmissions. However, it is only in the past two years that the American College of Surgeons National Surgical Quality Improvement Program database (ACS-NSQIP) specifies a reason for each readmission (described by International Classification of Disease, Ninth Edition [ICD-9] code). This is tracked for 30 days after surgery. As an initial effort to decrease readmissions, we identify the reasons for hospital readmission after lumbar posterior fusion.

Methods: Lumbar posterior fusions were identified in the 2012 and 2013 ACS-NSQIP datasets by Current Procedural Terminology code 22612. Complication ICD-9 codes were reviewed and grouped according to etiology and organ system. Data management and analyses were performed using Stata/IC 13.0.

Results: Of 5,267 lumbar posterior fusions, 229 patients $(4.35 \%)$ 


\begin{tabular}{|c|c|c|c|}
\hline \multirow{2}{*}{$\begin{array}{l}\text { READMISSION AFTER LUMBAR POSTERIOR FUSION } \\
\text { of } 5,267 \text { cases }\end{array}$} & \multicolumn{2}{|c|}{ readmitted } & \multirow{2}{*}{$\begin{array}{l}\text { per } 100 \\
\text { cases }\end{array}$} \\
\hline & $\#$ & $\%$ & \\
\hline TOTAL & 229 & 100 & 4.35 \\
\hline Surgical site & 167 & 72.9 & 3.17 \\
\hline $\begin{array}{l}\text { INFECTION } \\
\text { Deep (37), Superficial (25), Sepsis (9), Other (6) }\end{array}$ & 77 & 33.6 & 1.46 \\
\hline $\begin{array}{l}\text { OTHER SURGICAL SITE COMPLICATIONS } \\
\text { Seroma (17), Hematoma (14), Related to device (9) }\end{array}$ & 58 & 25.3 & 1.10 \\
\hline $\begin{array}{l}\text { PAIN ISSUES } \\
\text { Back pain (12), Pain, unspecified (8), Arthralgia (2) }\end{array}$ & 32 & 14.0 & 0.61 \\
\hline Thromboembolic & 17 & 7.4 & 0.32 \\
\hline PULMONARY EMBOLISM & 9 & 3.9 & 0.17 \\
\hline DEEP VENOUS THROMBOSIS & 8 & 3.5 & 0.15 \\
\hline Organ systems & 58 & 25.3 & 1.10 \\
\hline $\begin{array}{l}\text { RESPIRATORY SYSTEM } \\
\text { Pneumonia (8), Chest pain (2), bronchitis (1) }\end{array}$ & 13 & 5.7 & 0.25 \\
\hline $\begin{array}{l}\text { GENITOURINARY SYSTEM } \\
\text { Urinary tract infection (5) Acute kidney injury (1) }\end{array}$ & 9 & 3.9 & 0.17 \\
\hline $\begin{array}{l}\text { DIGESTIVE SYSTEM } \\
\quad \text { lleus (2), Intestinal obstruction (2), Diverticulitis (1) }\end{array}$ & 8 & 3.5 & 0.15 \\
\hline $\begin{array}{l}\text { CIRCULATORY SYSTEM } \\
\quad \text { Atrial fibrillation (1), Peripheral vascualr disease (1) }\end{array}$ & 4 & 1.7 & 0.08 \\
\hline $\begin{array}{l}\text { ENDOCRINE, NUTRITIONAL AND METABOLIC } \\
\text { Hypokalemia (1) }\end{array}$ & 1 & 0.4 & 0.02 \\
\hline ( & 6 & 2.6 & 0.11 \\
\hline Neuropacemaker status (2), syncope (1), fever (1) & & & \\
\hline Injury or poisoning & 5 & 2.2 & 0.09 \\
\hline Drug allergy (1), Drug-induced mental disorder (1) & & & \\
\hline
\end{tabular}

were readmitted within 30 days of surgery (Table 1). The most common reasons for readmission were surgical site infection $(77,1.46 \%$ of the posterior fusion cohort), other surgical site complications (58, $1.10 \%)$, and pain issues $(32,0.61 \%)$. Patients were readmitted less commonly for issues of other organ system issues, thromboembolic events, and miscellaneous other causes.

Conclusion: The most recent two years of ACS-NSQIP have allowed for assessment of readmissions after lumbar posterior fusion. In a time of increasing concern about hospital readmissions, it is identified that surgical site issues are the most common reason for readmission after these surgeries. Most of these were related to wound issues that may not be easily modifiable ( $2.56 \%$ of cases), but an evidence-based algorithm to minimize these events should be optimized. On the other hand, the third most common reason for readmission - pain $(0.61 \%$ of cases) - may be more modifiable. Further, ongoing analyses are being performed identify preoperative factors associated with readmission for pain issues in order to target such efforts.

Paper \# 19. The Effect of Weight Bearing on Non-operative Thoracolumbar Spine Fractures and Prognostic Variables of Sagittal Angle Deformation

Bryan Rynearson, BSN; Seth K. Williams, MD; Jason W. Savage, MD; Scott J. Hetzel, MS; Zachary Belford, B.S.; Paul A. Anderson, $M D$

\section{University of Wisconsin}

Study Design: Retrospective consecutive case series.

Objective: Determine the effect of weight bearing on deformation of non-operative spine fractures and identify predictive variables of angular change.

Summary of Background Data: Loss of normal sagittal alignment of the spine is a common sequela following thoracolumbar injuries. Patients treated non-operatively are especially at risk; however knowledge of the natural history of these injuries and predictors of deformation over time remains limited.

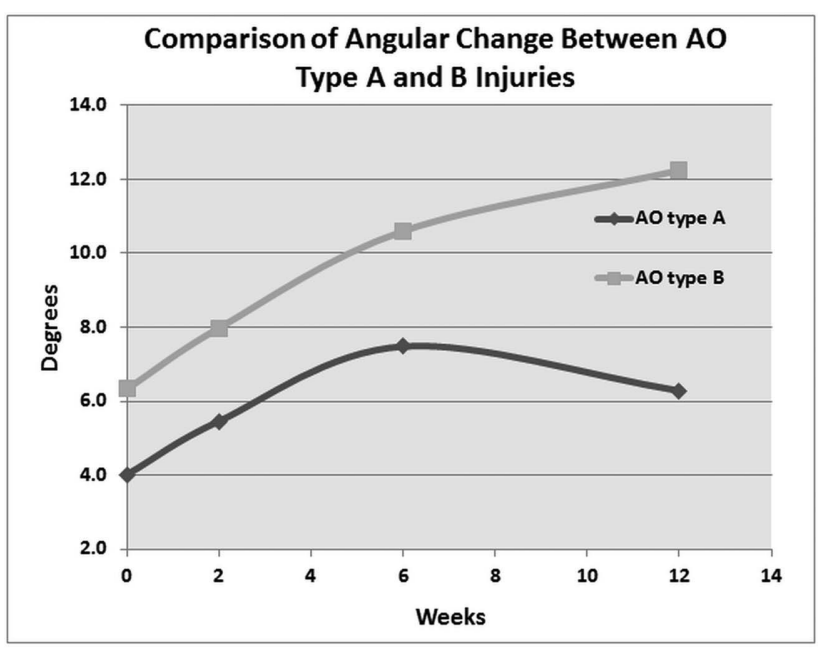

Methods: Patients with thoracolumbar fractures were identified via a trauma database. The angular change about the fracture site was determined comparing initial CT scan to upright radiographs over time. Measurements were correlated with initial fracture direction, region of injury, demographic data, and injury severity using the TLICS and AO classification systems.

Results: Ninety-nine eligible patients were included with a mean age of 45.8 years $( \pm 20.1)$. Overall, the average change initially was 4.5 degrees and at twelve weeks 7.8 degrees. AO type A fractures initially demonstrated 4.0 degrees of change that increased to 6.3 degrees by twelve weeks. Conversely, AO type B fractures had 6.3 degrees of change initially that increased significantly to 12.2 degrees by twelve weeks $(\mathrm{p}=0.039)$. Kyphotic fractures averaged +6.8 degrees of change initially versus -1.8 degrees for lordotic fractures $(\mathrm{p}=0.04)$ which increased to +9.3 degrees and -4.3 degrees, respectively by twelve weeks $(\mathrm{p}=0.09)$. TLICS and AO scores demonstrated a significant correlation with initial angular change (both $\mathrm{p}=0.015$ ) but only the AO score correlated significantly with the degree of angular change at twelve weeks $(\mathrm{p}=0.115$ and 0.035 respectively). Neither age, bone mineral density, or BMI had significant correlation with degree of angular change. Gender initially demonstrated a significant correlation with angular change with males averaging $2.0( \pm 5.7)$ degrees and females $4.5( \pm 4.1)$ degrees $(\mathrm{p}=0.02)$, however no significant difference existed at twelve weeks.

Conclusion: Non-operative thoracolumbar spine fractures undergo a predictable degree of collapse. Both direction and magnitude of deformation are strongly influenced by the initial sagittal alignment. Magnitude of collapse also correlates significantly with injury severity whereas gender, age, bone mineral density, and BMI do not appear to significantly impact the stability of these injuries.

Paper \# 20. The Influence of Spinopelvic Alignment on Development of Symptomatic Adjacent Level Disease Following Single Level Lumbar Fusion

Zachary J. Tempel, MD; Bryan D. Bolinger, DO; Gurpreet S. Gandhoke, MD; Phillip V. Parry, MD; David O. Okonkwo, MD PhD; Adam S. Kanter, MD

University of Pittsburgh Medical Center

Introduction: The annual incidence of developing symptomatic adjacent level disease (ALD) following lumbar fusion surgery ranges from 0.6 to $3.9 \%$. It has been suggested that sagittal malalignment may contribute to the development of ALD. We describe the relationship between spinopelvic parameters and symptomatic ALD 


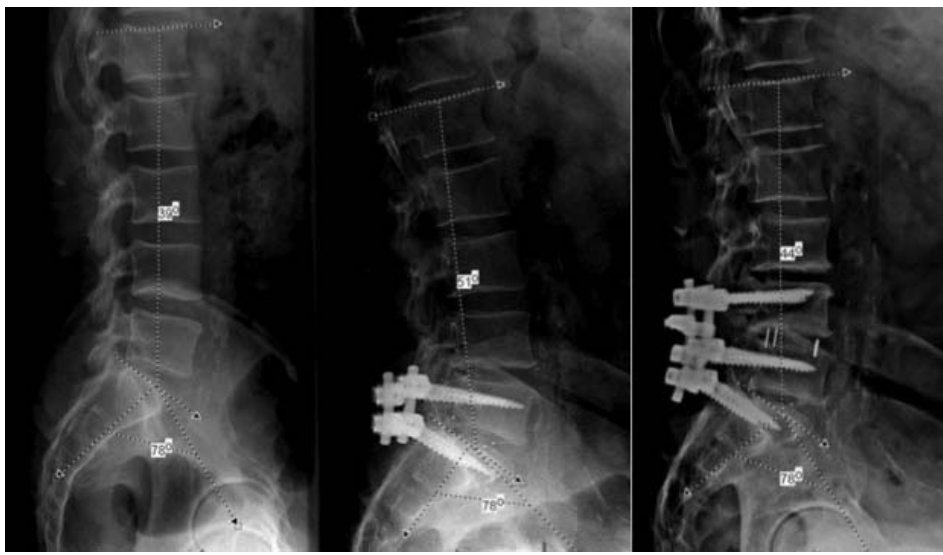

Figure 1. Preoperative (left) and postoperative (center) upright lumbar spine radiographs and MRI (right) of a 59 year-old male who underwent a L5/S1 TLIF. His preoperative and postoperative PI-LL mismatch were 39 and 27 degrees. Postoperative radiograph (right) following additional surgery for adjacent level disease demonstrates persistent PI-LL mismatch.

requiring revision surgery following single-level transforaminal lumbar interbody fusion (TLIF).

Methods: All patients who underwent a single-level TLIF at either L4/5 or L5/S1 at our institution for between July 2006 and December 2012 were analyzed for pelvic tilt (PT), sacral slope (SS), pelvic incidence (PI), lumbar lordosis (LL), and PI-LL mismatch. Using univariate and multivariate logistic regression analysis, we compared the spinopelvic parameters of patients who required revision surgery for symptomatic ALD against those who did not. The sensitivities and specificities for symptomatic ALD requiring surgery were based on preoperative values of PT $>24 \mathrm{o}$ and pelvic mismatch $>120$.

Results: 159 patients met inclusion criteria. The results of multivariate logistic regression analysis noted preoperative PI-LL mismatch (OR: 1.25 CI: 95\% $(1.16,1.33) \mathrm{p}<0.0001)$, postoperative PI-LL mismatch (OR: $1.40 \mathrm{CR}: 95 \%(1.27,1.54) \mathrm{p}<0.0001)$, and postoperative change in LL (OR: 0.96, CI: 95\% $(0.92,1.0) \mathrm{p}=0.39)$ to be statistically-significant independent predictors of symptomatic ALD requiring revision surgery. The sensitivity and specificity of a PI-LL mismatch $>120$ for symptomatic ALD requiring surgery following a single-level TLIF were $81.82 \%$ and $86.54 \%$. The positive predictive value for a postoperative PI-LL mismatch $=120$ for symptomatic ALD was $79.0 \%$; the area under the receiver operating characteristic (ROC) curve was 0.92 .

Conclusions: Abnormal preoperative spinopelvic parameters and persistent mismatch are associated with higher rates of symptomatic ALD requiring surgery following a single-level TLIF. Postoperative PI-LL mismatch was the strongest predictor. Accounting for spinopelvic parameters in the preoperative period may improve outcome following single-level TLIF.

Paper \#21. Diagnosing The Undiagnosed: Osteoporosis In Patients Undergoing Lumbar Fusion

Peter M. Formby, MD; Scott Wagner, MD; Daniel G. Kang, MD; Melvin D. Helgeson, MD

\section{Walter Reed National Military Medical Center}

Background: We utilized a recent technique utilizing Hounsfield Units (HU) from computed tomography (CT) scans to estimate bone mineral density (BMD) of the lumbar spine and hypothesized that this technique would reveal a high percentage of undiagnosed osteoporotic patients undergoing transforaminal lumbar interbody fusion.

Methods: A retrospective review of patients over age 50 under- going TLIF. The BMD of the lumbar spine was recorded if DEXA data were available. The average HU values of L4, measured on axial computed tomography (CT) scan, were also determined. Average HU values for patients with diagnosed lumbar osteoporosis (DEXA BMD $<0.75 \mathrm{~g} / \mathrm{cm} 2$ ) were then compared to those with osteopenia and normal BMD (between 0.75 to $0.9 \mathrm{~g} / \mathrm{cm} 2$ and $>0.9 \mathrm{~g} / \mathrm{cm} 2$, respectively). The percentage of patients with HU values consistent with osteoporosis, but without any formal evaluation, was also calculated.

Results: Over ten years, 143 patients over age 50 underwent TLIF, and 127 had available perioperative lumbar CT scans. Males and females comprised $60.6 \%$ and $39.4 \%$ of the population, respectively. Average age was 61.5 years (range: 50.0 to 83.5 years). Twenty nine patients had both DEXA and CT data available for analysis. There was a significant association with decreased HU in patients with lumbar BMD $<0.75 \mathrm{~g} / \mathrm{cm} 2(105.6 \mathrm{HU}+/-6.75 \mathrm{HU}, 95 \% \mathrm{CI})$ in comparison to patients with osteopenia $(146.0 \mathrm{HU}+/-4.1 \mathrm{HU}, \mathrm{p}=0.001)$ and with normal BMD $(164.8+/-22.3 \mathrm{HU}, \mathrm{p}=0.0001)$. Ten males $(13.0 \%)$ and 15 females $(38.3 \%)$ had L4 HU values consistent with osteoporosis. Fifty-three percent of females and $100 \%$ of males with osteoporotic HU values had never been formally evaluated for the disease.

Discussion: Our data suggest that $\mathrm{HU}$ can provide accurate assessment of bone mineral density of the lumbar spine, and that a high percentage of patients undergoing elective lumbar fusion have radiographic evidence of the disease.

Conclusions: We found that more than half of all females, and all males, over age 50 undergoing TLIF with osteoporosis of the lumbar spine were undiagnosed at the time of surgery. The clinical significance of this finding requires further study, but it is clear that increased vigilance on the part of the spine surgeon is required.

\section{Paper \# 22. Quality Of Life Following Acute Proximal Junctional Failure In Adult Spinal Deformity}

Matthew Colman, MD; E. Patrick Curry, MD; Prokopis Annis, MD; Ashley M. Neese, BS; William R. Spiker, MD; Brandon D. Lawrence, MD; Darrel S. Brodke, $M D$

\section{Midwest Orthopaedics at Rush}

Introduction: Acute proximal junctional failure (APJF) is a complication seen in 5-35\% of patients following surgery for adult spinal deformity. The incidence and risk factors for this condition have been defined, but less is known about patient reported outcomes following treatment.

Methods: Retrospective case-control study of 48 consecutive patients at a single institution with APJF. APJF was defined as fracture of the UIV or UIV+1, upper instrumentation failure, postoperative kyphosis $>15^{\circ}$, or need for revision surgery in the first 6 months after index surgery. Two cohorts were evaluated: APJF with non-operative treatment and APJF with operative treatment. To normalize the cohorts, we controlled for variables such as proximal junctional kyphosis angle, bone density, and medical comorbidities. Outcomes were assessed with the ODI, EQ5D, and back and leg pain VAS.

Results: 48 patients with APJF, mean follow-up 43 months, minimum follow-up 24 months, were split into 2 cohorts: 28 patients $(58 \%)$ in the non-operative group and 20 patients $(42 \%)$ in the operative group. At 2 years after developing APJF, the operativelytreated group had more back pain (VAS 5.7 vs. VAS 2.4, $\mathrm{p}=0.007$ ) and a trend toward more disability (ODI 46.4 vs. 31 .2, $\mathrm{p}=0.08$ ) compared with the non-operative group. In addition, 10 patients in the operative group had 11 further operative interventions (50\%) after being treated surgically for APJF compared to only 3 reiterative operations for non-APJF-related pseudoarthrosis (11\%) in the nonoperative group. Patients presenting with increased kyphosis $>15^{\circ}$ 
alone without fracture or instrumentation failure had significantly less back pain (VAS 1.4 vs. 4.1, $\mathrm{p}=0.05$ ) and better health-related quality-of-life (EQ5D 78.4 vs. 60.9, $\mathrm{p}=0.1$ ).

Conclusions: At 2-year follow-up, worse pain and disability were found in the operative group, which may be related to higher rates of reiterative operative intervention. The best outcomes were observed in a subset of APJF patients with proximal kyphosis alone and no fracture or instrumentation failure.

Discussion: Surgeons treating APJF following adult deformity surgery should consider maximizing conservative care, given the higher rates of reiterative surgery, pain, and disability following surgical correction of APJF.

Paper \# 23. Polishing Methods Less a Factor in Cell Adhesion than Surface Characteristics of Titanium Plasma Spray Coatings on PEEK

Byung Jo Victor Yoon, MS; Frank P. Cammisa, MD; Celeste Abjornson, $P h D$

\section{Hospital for Special Surgery}

Introduction: Titanium plasma spray coating on polyetheretherketone (PEEK) is a recent innovation to interbody spacer technology. Ti coating offers initial friction due to increased surface roughness and continued stability from the osteoconductivity on the device surface. The hydrophilic-osteophilic properties of commercially pure titanium $(\mathrm{CPTi})$ may provide an ideal environment promoting cell attachment and bony ongrowth when inserted into the interbody space against the bleeding endplate. An ideal coating must be defined as the surface material and topography directly affect cell adhesion. Zirconia polishing was previously reported in the dental
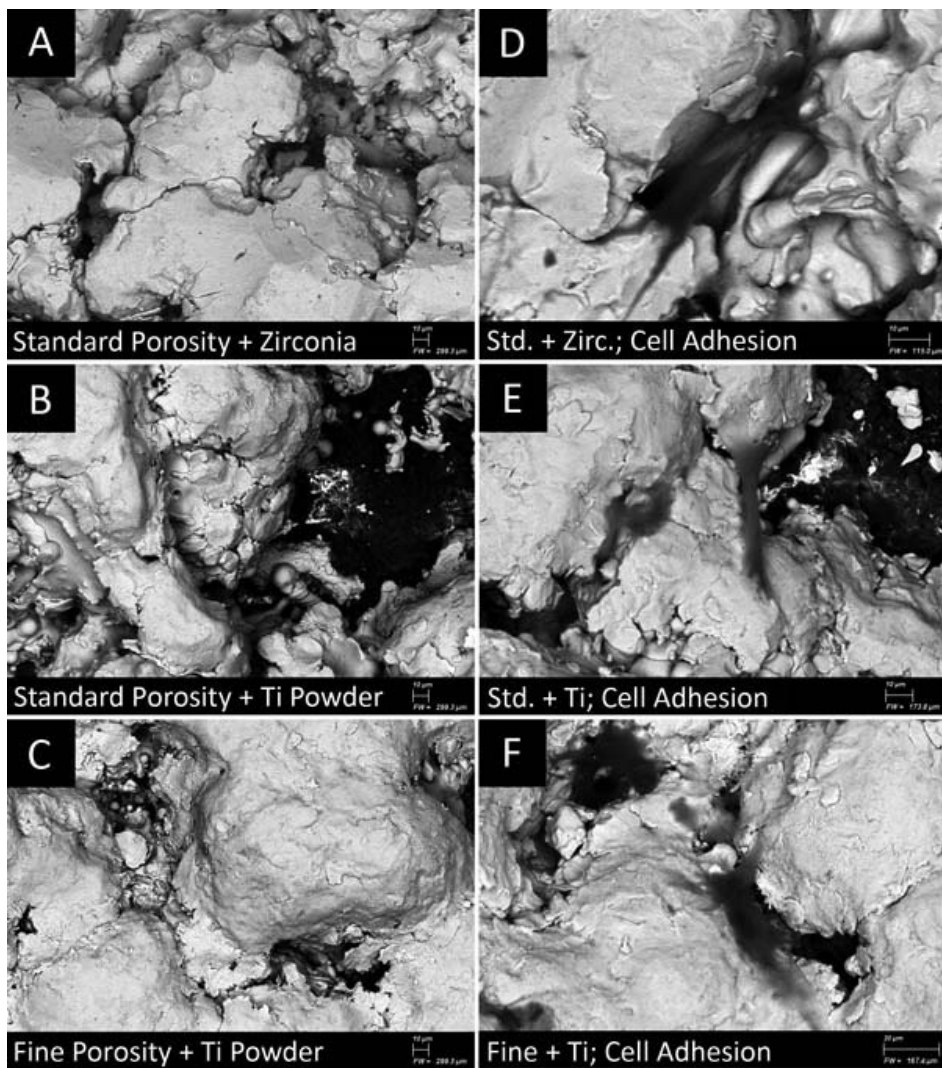

A) Std. with zirc. at $300 \mu \mathrm{m}$. B) Std. with Ti at $300 \mu \mathrm{m}$. C) Fine with Ti at 300 $\mu \mathrm{m}$. D) Cells on std. with zirc. E) Cells on std. with Ti. F) Cells on fine with Ti. literature may increase the osteogenic response to the surface. The purpose of the study is to determine the relationship between porosity/topography and cell attachment and further whether polishing methods affect cell adhesion to the surface. We compared cell adhesion of two different polishing methods on the same topography/ porosity and one polishing method at two different topographies/ porosities.

Methods: A porous CPTi coating was plasma sprayed onto the PEEK substrate in controlled atmosphere. Studied groups are: 1 standard CPTi coating with zirconia polishing, 2 - standard CPTi coating with CPTi polishing, and 3 - fine CPTi coating with CPTi polishing. Coating thickness, porosity and surface roughness were characterized using an optical microscope. Human mesenchymal stem cells (hMSCs) were seeded onto CPTi coated PEEK substrate at an initial density of 25,000 cells $/ \mathrm{cm} 2$, cultured for 24 hours, and fixed in $10 \%$ formalin. The cultured hMSCs were visualized by 4',6-diamidino-2-phenylindole (DAPI) staining. Samples were imaged using an environmental SEM.

Results: Image analysis of the CPTi coatings showed uniform and rough surfaces. hMSCs showed 2 fold increase in adhesion and greater pseudopodia extensions on Group 3 (214.008 cells $/ \mathrm{mm} 2)$ relative to Groups 1 and 2. No difference in cell adhesion was found between Groups 1 and 2 (89.494 cells/mm2, 101.167 cells $/ \mathrm{mm} 2)$.

Conclusions: Previously, it was accepted without much scrutiny that surface coatings were beneficial. This study begins to discover that surface topography has greater effect on cell adhesion and proliferation than material composition, and may lead to greater surgical efficacy.

Paper \# 24. Dioxin Exposure Inhibits Osteogenic Differentiation and Impairs Bone Healing in a Rat Spine Fusion Model

Wellington K. Hsu, MD; Erin L. Hsu, PhD; Sean M. Mitchell, BS; Abhishek S. Kannan, BS; Chawon Yun, Ph.D.; Kevin A. Sonn, MD; Sharath S. Bellary, MD; Sohaib Z. Hashmi, MD; Marco C. Mendoza, MD; Michael S. Nickoli, MD; John T. Nelson, MD; Jason H. Ghodasra, M.D., M.S.C.I.; Christian Park, BS; Anjan Ghosh, BS; Akshay Jain, BS; Harry T. Mai, $B S, B A ;$ Stuart R. Stock, PhD

\section{Northwestern University Department of Orthopaedic Surgery}

Introduction: Smoking inhibits bone healing and leads to increased rates of pseudoarthrosis. We found previously that dioxin, a toxic constituent of cigarette smoke that activates the Aryl hydrocarbon receptor (Ahr) pathway, inhibits spinal fusion in a rat posterolateral fusion (PLF) model. We hypothesized that prolonged cessation of dioxin exposure would alleviate this effect in vivo, and that co-treatment of bone marrow stromal cells (BMSC) with Ahr antagonists in vitro would counteract the inhibitory effects of dioxin on osteogenic differentiation.

Methods: Rats were treated with dioxin or vehicle for 6 weeks, followed by PLF across the L4-L5 transverse processes using rhBMP-2/absorbable collagen sponge. A third treatment group also received dioxin injections, followed by cessation of treatment for 4 half-lives prior to PLF procedure. After euthanasia at 4 weeks post-op, spines were evaluated using radiographs, microCT, and fusion scoring via manual palpation. To determine whether Ahr antagonists mitigate the effects of dioxin on osteogenic differentiation, BMSC were harvested from rats and treated with vehicle, dioxin, or dioxin+antagonists, followed by quantitation of ALP activity, cell migration rate, and mineralization capacity.

Results: Cessation of dioxin exposure reversed its negative affects on fusion rates ( $100 \%$ vs $80 \%$, control vs dioxinrecovery groups, $\mathrm{p}=0.20$ ), but not fusion scores ( 1.75 vs 0.97 ; $\mathrm{p}<0.01)$. In vitro, co-treatment with $\mathrm{Ahr}$ antagonists rescued 
Figure 1. (A) Fusion scores for DMS0, dioxin, and dioxin

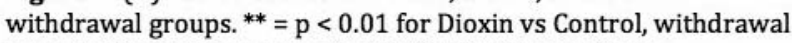
vs control (B) Fusion rates for DMSO, dioxin, and dioxin withdrawal groups. ${ }^{* *}=p<0.01$ Control vs dioxin treatment

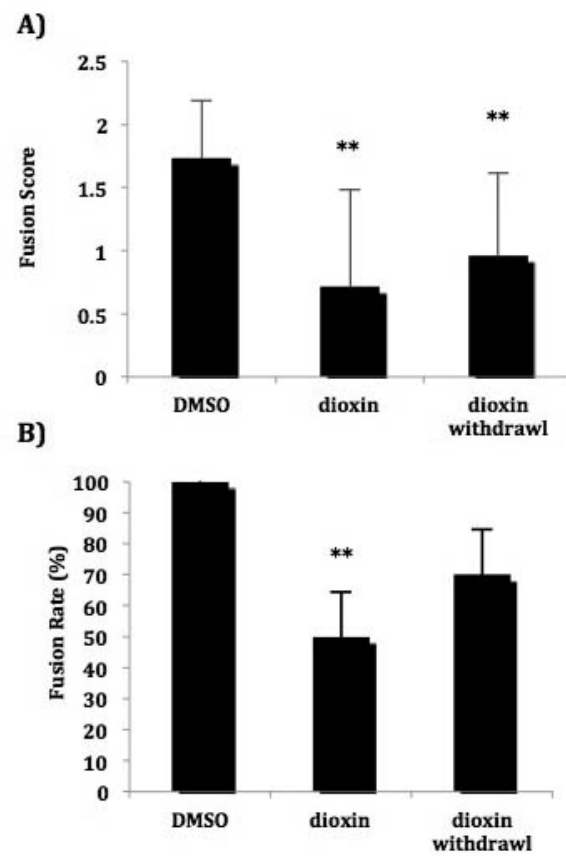

the inhibitory effect of dioxin on ALP activity, cell migration rate, and mineralization.

Conclusion: Since cessation of dioxin exposure facilitated a partial recovery of bone healing capacity, our data suggest that the Ahr pathway is a therapeutic target to combat the effects of dioxin on bone. Future studies will quantify the capacity of Ahr antagonists to protect against dioxin action in vivo, with the ultimate goal of identifying a therapeutic approach to improve bone healing in smokers.

Paper \# 25. Large Animal Model Development for Use in Testing a Novel Cell Therapy for Degenerative Disc Disease

Lara I. Silverman, PhD; Antwain Howard, DVM; Kevin Foley, MD

Semmes-Murphey Neurologic Institute

Introduction: Human testing of spinal therapies requires demonstrating robust safety and efficacy in a large animal model. For degenerative disc disease, the intervertebral discs of large animals must be injured in such a way to mimic key aspects of the human degenerative condition. However, there is currently no consensus on the best method to employ or species to select in order to achieve this goal. Therefore, the objective of this work was to optimize a large animal model suitable for toxicological assessment of our novel cell therapy.

Methods: In a series of studies, Gottingen minipigs were anesthetized and the lumbar spine surgically accessed. Fluoroscopic guide $(\mathrm{C}$-arm) was used to ensure proper needle placement along two planes of view. Various injury techniques were performed, and the wounds closed. Animals were monitored for 4-6 weeks to assess radiographic changes to disc height as well as histological appearance after decalcification and paraffin embedding.

Results: A single stab with aspiration was used to remove NP, but showed limited decrease in disc height. Next, NP was removed from each disc three times with sequential stab/aspirations, each separated by $1-3 \mathrm{~mm}$. While the method resulted in consistent decrease in disc height $(65 \%$ Stdev $2.7 \%$ compared to $100 \%$ original disc height after 4 weeks, $n=8$ ), the histological appearance of the injury varied considerably, including significant damage to the annulus. Finally, an $18 \mathrm{G}$ guide needle was placed into the annulus, and another $22 \mathrm{G}$ needle fed into the guide needle three separate times and aspirated with a small syringe, which resulted in removal of 20-40 mg of NP (compared to 2-6 mg with single stab/aspirate and large syringe). The resulting disc height was consistent (71\% StDev 5.2 after 2 weeks, $72 \%$ StDev 6.7 after 6 weeks, n=9), as well as the histological appearance.

Conclusions: A novel technique was developed to create a degeneration-like injury in minipigs. This model can now be used to establish the safety profile and route of administration of a novel cell therapy that will support an Investigational New Drug application to the FDA.

Paper \#26. Mesenchymal Stem Cell Survival, Proliferation and Migration in a Murine Model of Lumbar Posterolateral Spinal Fusion

Christina Holmes, PhD; Ioan Lina, BS; Jason Liauw, M.D.; ShengFu Lo, MD; Benjamin Elder, MD., PhD; Debebe Theodros, BS; Timothy Witham, MD

Johns Hopkins University School of Medicine

Introduction: Clinically, bone marrow aspirate, which contains mesenchymal stem cells, has been successfully used alongside a variety of grafting materials to augment spinal fusion procedures [Neen et al, 2006, Spine (Phila Pa 1976). 31(18):E636-40; Carter et al, 2009, Spine J. 9(6):434-8]. To date, studies evaluating these procedures have focused on radiographic monitoring and end-point analysis. Little is known about the survival and proliferation of transplanted mesenchymal cells in the context of spinal fusion or how much they contribute to bone formation compared to host cells from adjacent decorticated bone. Insights into these cell dynamics will give us a deeper understanding of the biological processes underlying spinal fusion and allow for evaluation and development of fusion therapies that reduce rates of fusion failure, or pseudoarthrosis.

Materials and Methods: Forty FVB/NJ mice underwent posterolateral lumbar spinal fusion surgery and were divided into 4 groups based upon the fusion graft employed: syngeneic iliac crest bone graft $(n=10)$, Vitoss $\AA$ bone-matrix alone $(n=10)$, Vitoss $\AA$ bonematrix with syngeneic bone marrow cells $(2.5 \times 106$ cell/side) $(n=$ $10)$, and Vitoss ${ }^{\circledR}$ bone-matrix with transgenic luciferase-expressing syngeneic bone marrow cells $(2.5 \times 106$ cell/side $)(n=10)$. Bone marrow cells were isolated from the long bones of either syngeneic $\mathrm{FVB} / \mathrm{NJ}$ mice or syngeneic FVB mice which constitutively express the luciferase gene (FVB-Tg(CAG-luc,-GFP)L2G85Chco/J mice). Bioluminescence imaging was performed at one, two, four and six weeks, at which point the animals were euthanized.

Results: Bioluminescent imaging data of mice transplanted with syngeneic luciferase-expressing iliac crest bone graft demonstrated quantifiable cellular viability as early as 24 hours following surgery. Animals imaged at later time points exhibited increased luminescence signals.

Conclusions: We have demonstrated a novel murine model which enables non-invasive in vivo monitoring of implanted transgenic luciferase-expressing bone marrow cells following spinal fusion surgery. Future applications of this model will include the assessment of additional therapeutics for improving spinal fusion in translational research studies. 
Paper \# 27. Of 12,567 Lumbar Discectomies, 2.47\% Of Patients Readmitted Within 30 Days: Pain Issues The Most Common Reason For Readmission

Matthew L. Webb, AB; Adam M. Lukasiewicz, MSc; Andre M. Samuel, BBA; Daniel D. Bohl, MPH; Bryce A. Basques, BS; Arya G. Varthi, M.D.; Jonathan Newman Grauer, MD

Yale School of Medicine

Background: The Affordable Care Act codifies penalties for hospital readmissions. Large clinical outcomes databases can be used to quantify postoperative complications and readmissions. However, it is only in the past two years that the American College of Surgeons National Surgical Quality Improvement Program database (ACS-NSQIP) specifies a reason for each readmission (described by International Classification of Disease, Ninth Edition [ICD-9] code). This is tracked for 30 days after surgery. As an initial effort to decrease readmissions, we identify the reasons for hospital readmission after lumbar discectomy.

Methods: Lumbar discectomies were identified in the 2012 and 2013 ACS-NSQIP datasets by Current Procedural Terminology code 63030. ICD-9 codes were reviewed and grouped according to etiology and organ system. Data management and analyses were performed using Stata/IC 13.0.

Results: Of 12,567 lumbar discectomies, 311 patients $(2.47 \%)$ were readmitted within 30 days of surgery (Table 1$)$. The most common reasons for readmission were pain issues $(98,0.78 \%$ of the discectomy cohort), surgical site infection $(85,0.68 \%)$, and other surgical site complications $(45,0.36 \%)$. Patients were readmitted less commonly for thromboembolic events, other organ system issues, and other causes specified and unspecified.

Conclusion: The most recent two years of ACS-NSQIP have

\begin{tabular}{|c|c|c|c|}
\hline \multirow{2}{*}{$\begin{array}{l}\text { READMISSION AFTER LUMBAR DISCECTOMY } \\
\text { of } 12,567 \text { cases }\end{array}$} & \multicolumn{2}{|c|}{ readmitted } & \multirow{2}{*}{$\begin{array}{l}\text { per } 100 \\
\text { cases }\end{array}$} \\
\hline & $\#$ & $\%$ & \\
\hline TOTAL & 311 & 100 & 2.47 \\
\hline Surgical site & 228 & 73.3 & 1.81 \\
\hline $\begin{array}{l}\text { PAIN ISSUES } \\
\text { Pain, unspecified (18), Back pain (15), Radiculitis (6) }\end{array}$ & 98 & 31.5 & 0.78 \\
\hline $\begin{array}{l}\text { INFECTION } \\
\text { Deep (44), Superficial (22), Sepsis (13), Other (6) }\end{array}$ & 85 & 27.3 & 0.68 \\
\hline $\begin{array}{l}\text { OTHER SURGICAL SITE COMPLICATIONS } \\
\text { Hematoma (10), Wound disruption (8), Dural tear (5) }\end{array}$ & 45 & 14.5 & 0.36 \\
\hline Thromboembolic & 32 & 10.3 & 0.25 \\
\hline DEEP VENOUS THROMBOSIS & 17 & 5.5 & 0.14 \\
\hline PULMONARY EMBOLISM & 15 & 4.8 & 0.12 \\
\hline Organ systems & 37 & 11.9 & 0.29 \\
\hline $\begin{array}{l}\text { DIGESTIVE SYSTEM } \\
\text { Intestinal obstruction (2), Functional disorder (1) }\end{array}$ & 8 & 2.6 & 0.06 \\
\hline $\begin{array}{l}\text { RESPIRATORY SYSTEM } \\
\text { Pneumonia (5), Chest pain (2), other (1) }\end{array}$ & 8 & 2.6 & 0.06 \\
\hline $\begin{array}{l}\text { GENITOURINARY SYSTEM } \\
\text { Rentention of urine (3), Hyperplasia of prostate (1) }\end{array}$ & 7 & 2.3 & 0.06 \\
\hline $\begin{array}{l}\text { CIRCULATORY SYSTEM } \\
\text { Subdural hemorrhage (1), CVA* (1), Hypotension (1) }\end{array}$ & 7 & 2.3 & 0.06 \\
\hline $\begin{array}{l}\text { CENTRAL NERVOUS SYSTEM (unrelated to surgical site) } \\
\text { Other, unspecified (3), Migraine (1) }\end{array}$ & 4 & 1.2 & 0.03 \\
\hline $\begin{array}{l}\text { ENDOCRINE, NUTRITIONAL AND METABOLIC } \\
\text { Dehydration (1), Diabetes (1) }\end{array}$ & 2 & 0.6 & 0.02 \\
\hline $\begin{array}{l}\text { SKIN AND SUBCUTANEOUS TISSUE } \\
\text { Cellulitis of the leg (1) }\end{array}$ & 1 & 0.3 & 0.01 \\
\hline Other & 9 & 2.9 & 0.07 \\
\hline Syncope (3), Fever (2), Altered mental status (1) & & & \\
\hline Injury or poisoning & 5 & 1.6 & 0.04 \\
\hline Vertebral fracture (1), Fall (1), Analgesics (1) & & & \\
\hline
\end{tabular}

${ }^{*} \mathrm{CVA}=$ cerebrovascular accident allowed for assessment of readmissions after lumbar discectomy. In a time of increasing concern about hospital readmissions, it is identified that postoperative pain, which should be controllable, is the most common reason for readmission after this procedure. It is thus clear that increased focus needs to be placed on optimizing postoperative pain control. Further, ongoing analyses are being performed identify preoperative factors associated with readmission for pain issues in order to target such efforts.

Paper \# 28. Use of Neuropathic Pain Questionnaires in Predicting the Development of Failed Back Surgery Syndrome following Lumbar Discectomy for Radiculopathy

Mohammed F. Shamji, MD, PhD; Alina Shcharinsky, RN(EC), $M N$, $\mathrm{CNN}(\mathrm{C})$

\section{Toronto Western Hospital}

Objective: Failed back surgery syndrome (FBSS) is a type of neuropathic pain where extremity symptoms persist despite structurally corrective spinal surgery. This implies more substantial nerve damage rather than simply dysfunction whereby correcting the inciting structural derangement does not provide for clinical resolution. What remains unclear is how to predict which patients are more or less likely to derive benefit from surgical intervention, and whether specific pain characteristics are associated with different likelihoods of good outcome.

Methods: This study analyzed a prospective dataset of painful radiculopathy patients assessed and surgically managed for radiculopathy secondary to lumbar degenerative spondylosis by the senior author at Toronto Western Hospital neurosurgery over the last 24 months. Clinical parameters to be analyzed include general demographic information, preoperative and postoperative clinical examination, surgical details, general self-reported pain and disability scores, and neuropathic pain magnitude and scores. The two screening tests used in this study were the Douleur Neuropathique 4 (DN4) and Leeds Assessment of Neuropathic Symptoms and Signs (LANSS), with correlation tested for both absolute ordinal score as well as screen positivity for neuropathic pain. Multiple logistic regression analysis ( 0.05 level of significance) was used to define predictors of persistent postoperative symptomatology.

Results: Among 250 patients treated for lumbar radiculopathy, $12 \%$ were classified as having FBSS with modest relief of leg pain compared with the cohort of patients in whom substantial improvement was seen. The postoperative condition was highly assosciated with abnormal preoperative screens for neuropathic pain (Figure 1 ), but not gender, smoking status, or preoperative pain severity (multiple logistic regression, $\alpha=0.05$ ). Good correlation was seen between the two screening test used in this study for both absolute ordinal score (Spearman's $Q=0.84, p<0.001$ ) and thresholding for terming the patient as having neuropathic pain features (Spearman's $\mathrm{Q}=0.48, \mathrm{p}<0.001)$.

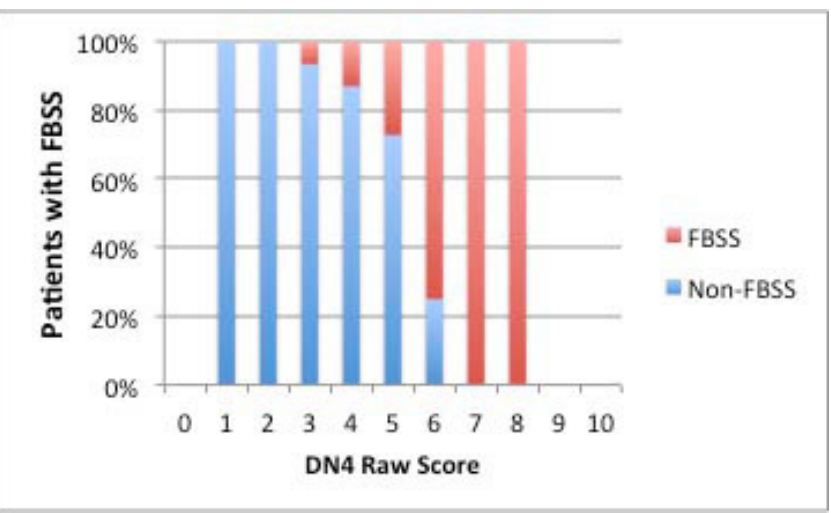


Conclusion: While FBSS was more common among younger and female patients, this still occurred with low overall frequency in this population. Higher neuropathic pain screening scores correlated strongly with the likelihood and severity of significant postoperative leg pain. Further work is required to develop more accurate prognostication tools for patients undergoing structural spinal surgery for lumbar radiculopathy.

Paper \# 29. Risk Factors for Venous Thromboembolism following Thoracolumbar Surgery: Analysis of 43,777 patients from ACS-NSQIP 2005-2012

Arjun S. Sebastian, MD; Sanjeev Kakar, MD; Amy Wagie, B.A.; Elizabeth Habermann, Ph.D.; Bradford L. Currier, MD; Ahmad Nassr, MD

\section{Mayo Clinic}

Introduction: The incidence of venous thromboembolism (VTE) following spine surgery is poorly understood. Rates of VTE following spine surgery vary from $0.3 \%-31 \%$. American College of Surgeons National Surgical Quality Improvement Project (ACSNSQIP) is a validated database that collects thirty day complication data from over 400 institutions. We report a comprehensive examination of the incidence, timing, and risk factors for VTE following thoracolumbar surgery.

Methods: The ACS-NSQIP Participant Use Data File identified 43,777 patients who underwent thoracolumbar surgery from 20052012. Patient demographics, comorbidities, laboratory values, and operative factors were identified. The incidence and timing of deep vein thrombosis (DVT) and pulmonary embolus (PE) were determined. Univariate testing and multivariable analysis was performed to identify significant predictors.

Results: Of the 43,777 patients identified has having had thoracolumbar surgery, the most frequent procedure was posterior decompression $(28,529,65.2 \%)$. Overall, 202 cases of PE $(0.5 \%)$ and 311 cases of DVT $(0.7 \%)$ were identified. VTE rates were highest in patients undergoing corpectomy. The mean time for diagnosis was $9.4 \pm 7.3$ days for PE and 10.3 \pm 7.4 for DVT. (Table 1) In 2011$2012,28.0 \%$ of patients with VTE were readmitted. Predictors of VTE were identified in univariate analysis. (Table 2) Independent risk factors for VTE included length of stay 6 days or greater (OR 4.07, p < .001), cancer (OR 1.77, p < .001), WBC > 12 (OR 1.76, p $<.001)$, paraplegia (OR 1.75, $\mathrm{p}=.003)$, albumin $<3(\mathrm{OR} 1.73 \mathrm{p}=$ $.012)$, ASA class 4 or greater (OR 1.54, $\mathrm{p}=.035), \mathrm{BMI}>40(\mathrm{OR}$ $1.49, \mathrm{p}=.030)$, and operative time greater than 193 minutes (OR $1.43, \mathrm{p}=.050)$. Length of stay less than three days was protective (OR .427, $\mathrm{p}<.001)$. In the spinal deformity cohort, bleeding disorders were predictive of VTE (OR 5.86, $\mathrm{p}=.008$ ).

\begin{tabular}{|c|c|c|c|c|c|}
\hline Procedure & $N$ & PE rate & Days until PE & DVT rate & Days until DVT \\
\hline Total & 43,777 & $202(0.5 \%)$ & $9.4 \pm 7.3$ & $311(0.7 \%)$ & $10.3 \pm 7.4$ \\
\hline $\begin{array}{l}\text { Posterior } \\
\text { Decompression }\end{array}$ & 28,529 & $98(0.3 \%)$ & $9.7 \pm 7.5$ & $149(0.5 \%)$ & $10.5 \pm 7.6$ \\
\hline Posterior Fusion & 6746 & $47(0.7 \%)$ & $10.4 \pm 8.1$ & $70(1.04 \%)$ & $11.0 \pm 7.2$ \\
\hline PLIF/TLIF* & 3689 & $22(0.6 \%)$ & $7.7 \pm 5.8$ & $29(0.8 \%)$ & $7.9 \pm 6.4$ \\
\hline $\mathrm{AlF}^{* *}$ & 2179 & $13(0.6 \%)$ & $7.2 \pm 5.4$ & $12(0.6 \%)$ & $9.8 \pm 5.4$ \\
\hline Corpectomy & 474 & $8(1.7 \%)$ & $12.4 \pm 8.1$ & $18(3.8 \%)$ & $8.9 \pm 7.5$ \\
\hline Oncologic & 920 & $5(0.5 \%)$ & $8.8 \pm 6.4$ & $22(2.4 \%)$ & $12.6 \pm 8.6$ \\
\hline $\begin{array}{l}\text { Transpedicular } \\
\text { decompression }\end{array}$ & 823 & $7(0.9 \%)$ & $6.0 \pm 5.3$ & $8(1.0 \%)$ & $7.9 \pm 6.7$ \\
\hline $\begin{array}{l}\text { Lateral extracavitary } \\
\text { arthrodesis }\end{array}$ & 194 & $1(0.5 \%)$ & $14.0 \pm$ & $2(1.0 \%)$ & $15.0 \pm 1.4$ \\
\hline Disc Arthroplasty & 120 & $1(0.8 \%)$ & $6.0 \pm$ & $1(0.8 \%)$ & $6.0 \pm$ \\
\hline Spinal Deformity & 1138 & $11(1.0 \%)$ & $9.5 \pm 7.3$ & $20(1.8 \%)$ & $10.6 \pm 7.3$ \\
\hline Anterior Osteotomy & 312 & $1(0.3 \%)$ & $9.0 \pm$ & $5(1.6 \%)$ & $10.2 \pm 5.2$ \\
\hline Posterior Osteotomy & 209 & $2(1.0 \%)$ & $2.5 \pm 3.5$ & $11(1.8 \%)$ & $11.3 \pm 7.1$ \\
\hline
\end{tabular}

Conclusions: We report an overall thirty day VTE rate of $1.2 \%$. Patients undergoing corpectomy were at highest risk for VTE. Patients with longer hospital admissions had a several fold increase in VTE risk. Further studies are needed to develop algorithms to stratify VTE risk and direct prophylaxis accordingly.

\section{Paper \# 30. A Survey On Spine Surgeons' Opinions On The Release Of The CMS Data}

John Koerner, MD; Gregory D. Schroeder, MD; Anuj Shah, BS; Paul M. Arnold, M.D.; Robert E. Isaacs, MD; Alexander R. Vaccaro, MD, PhD; Kris Radcliff, MD

The Rothman Institute at Thomas Jefferson University

Summary of Background Data: In April 2014 the Centers for Medicare and Medicaid Services (CMS) released a dataset for the public which included information on services provided by physicians and healthcare providers for Medicare beneficiaries in the 2012 calendar year. The objective of this study is to determine spine surgeons' opinions on the release of the CMS data, and determine how they feel this information may ultimately affect patient care.

Methods: A survey was sent to members of the Association for Collaborative Spine Research (ACSR) regarding their practice patterns and opinions on the release of the CMS data. Determinants included surgical subspecialty, practice setting, years in practice and region. The average response was collected for each question and compared across groups. Additionally, questions in which greater than $75 \%$ of respondents either agreed (agree or strongly agree) or disagreed (disagree or strongly disagree) were identified.

Results: Seventy-six surgeons completed the survey, and while the overall interobserver reliability between each question was only slight $(x=0.11)$, more than $75 \%$ of respondents either agreed or strongly agreed with five statements and, more than $75 \%$ of respondents either disagreed or strongly disagreed with six statements (Table 1). While $86 \%$ of surgeons are in favor of more transparency, $83 \%$ of respondents felt that without the proper context, the data released does not accurately portray spine surgery. Additionally, $96 \%$ of spine surgeons do not believe the CMS data will help patients decide which spine surgeon is best for them.

Conclusions: Spine surgeons are in favor of more transparency in healthcare, but they do not feel the release of the CMS data either accurately represents spine surgeons or will help patients better identify the appropriate surgeon. In spite of these concerns, it is

\begin{tabular}{|c|c|}
\hline $\begin{array}{c}\text { Questions in which }>\mathbf{7 5 \%} \text { of respondents agreed or strongly } \\
\text { agreed }\end{array}$ & $\begin{array}{l}\text { Percent who } \\
\text { agree or } \\
\text { strongly agree }\end{array}$ \\
\hline $\begin{array}{l}\text { Despite the limited compensation I ultimately receive from treating } \\
\text { Medicare patients, I feel a moral obligation to treat these patients. }\end{array}$ & $84.60 \%$ \\
\hline $\begin{array}{l}\text { Physicians at tertiary referral centers will be viewed as more aggressive in } \\
\text { performing fusion and complex fusion without information such as } \\
\text { comorbidities and percent of cases that are referred revisions. }\end{array}$ & $76.90 \%$ \\
\hline $\begin{array}{l}\text { I am in favor of more transparency in quality and in management of } \\
\text { healthcare costs. }\end{array}$ & $86.20 \%$ \\
\hline $\begin{array}{l}\text { Without data on long-term cost of care, the CMS data does not represent } \\
\text { spine surgery accurately because there are high initial expenditures. }\end{array}$ & $83.10 \%$ \\
\hline $\begin{array}{l}\text { Because of the complexity of this database, patients are less likely to use } \\
\text { this data, and it is more likely to be used by special interest groups and } \\
\text { malpractice attorneys }\end{array}$ & $78.50 \%$ \\
\hline $\begin{array}{c}\text { Questions in which }>\mathbf{7 5 \%} \begin{array}{c}\text { of respondents disagreed or strongly } \\
\text { disagreed }\end{array}\end{array}$ & $\begin{array}{l}\text { Percent who } \\
\text { disagree or } \\
\text { strongly } \\
\text { disagree }\end{array}$ \\
\hline $\begin{array}{l}\text { The intentions of the CMS to make our healthcare system more transparent, } \\
\text { affordable, and accountable were fulfilled by the release of this information. }\end{array}$ & $76.80 \%$ \\
\hline $\begin{array}{l}\text { The CMS data will help patients decide which spine surgeon is best for } \\
\text { them. }\end{array}$ & $95.70 \%$ \\
\hline $\begin{array}{l}\text { The availability of this data will help patients identify surgeons with } \\
\text { inappropriate indications. }\end{array}$ & $84.10 \%$ \\
\hline Articles in the press presented this data in an accurate way. & $84.10 \%$ \\
\hline $\begin{array}{l}\text { It is important for patients to know the percent of Medicare patients I end } \\
\text { up performing a spinal fusion on. }\end{array}$ & $78.50 \%$ \\
\hline $\begin{array}{l}\text { I have stopped treating Medicare patients because of the release } \\
\text { data. }\end{array}$ & $95.30 \%$ \\
\hline
\end{tabular}


unlikely that the release of the CMS data will significantly impact the accessibility of a spine surgeon to a Medicare beneficiary.

\section{Paper \# 31. Radiographic Fate Of S2-Alar-Iliac Screws After 12-month Minimum Follow-up}

Marcus D. Mazur, MD; Mark A. Mahan, MD; Andrew T. Dailey, $M D$

\section{University of Utah}

Introduction: Lumbopelvic fixation with S2-alar-iliac (S2AI) screws is a recently developed technique used to improve fusion rates. The S2AI screw crosses the cortical surfaces of the sacroiliac joint with the tip positioned in the dense bone overlying the sciatic notch. This trajectory increases screw purchase, which may improve its biomechanical strength. We report radiographic outcomes in patients who underwent lumbopelvic fixation with S2AI screws with a minimum 12-month follow-up.

Methods: A retrospective review was conducted at the University of Utah of adult patients who underwent lumbopelvic fixation with S2AI screws. Patients underwent CT scans both preoperatively and postoperatively at 12 months or longer. CT scans were reviewed to determine: S2AI screw backout or breakage, peri-screw lucency, sacroiliac joint fusion status and L5-S1 fusion status. Radiographic fusion was defined as the presence of solid bony bridging across cortical surfaces.

Results: Postoperative CT evaluation of 26 S2AI screws was performed on 13 patients [mean follow-up 24.8 months (14 to 52 months)]. Nine patients had L5-S1 interbody grafts. Evidence of loosening was identified in 5 S2AI screws (19\%) in 3 patients $(23 \%)$. Screw lucency was partial, occurring only at the tip in the iliac region in 3 screws and in the sacral region in 2 screws. No lucency involved the full screw length (both sacral and iliac regions). L5-S1 nonunion was seen in 1 patient (8\%), who had evidence of bilateral screw loosening of the sacral regions. Two patients with screw loosening had L5-S1 fusion. No patients had fusion across the sacroiliac joint, developed screw backout or breakage, or required reoperation for L5-S1 nonunion or S2AI-screw related complication.

Conclusion: Patients with S2AI screws demonstrated a high rate of L5-S1 fusion (92\%), no evidence of sacroiliac joint fusion, no major screw-related complications, and low rates of partial periscrew lucency.

\section{Paper \#32. Finding the Right Fit: Studying the Effect of Tap} Design on Screw Pullout Strength

Ehsan Jazini, MD; Mark Moldovsky, MS; Oliver Tannous, MD; Brandon Bucklen, PhD; Joseph R. O'Brien, MD, MPH; steven c. ludwig, MD

\section{University of Maryland Medical Center}

Introduction: Compromise of pedicle screw purchase and screw pullout is a major concern in maintaining rigid fixation in spinal surgery especially when bone quality is compromised as in the case of osteoporosis. Various tapping techniques are employed by surgeons who undertap with little consistency between manufacturers or techniques. Specifically, we questioned whether a mismatch between tapping dimensions (pitch, depth) and the intended screw dimensions affected the biomechanics, and hypothesized that it did not.

Methods: Foam blocks with dimensions of $40 \mathrm{~mm} \times 40 \mathrm{~mm} \times$ $50 \mathrm{~mm}(\mathrm{n}=105)$ and $10 \mathrm{lb} / \mathrm{ft}^{3}$ density were used to model osteoporotic bone. Each block was perforated with an awl and then probed to a depth of $40 \mathrm{~mm}$, perpendicular to the plane of the block. Next, the blocks were tapped using custom made taps of varying thread depth and pitch to a depth of $40 \mathrm{~mm}$. The blocks were sorted into 7 groups: 1) probe only, 2) [0.5mm D, 1.5mm P], 3) [0.5 $\mathrm{mm} \mathrm{D}, 2 \mathrm{~mm}$ $\mathrm{P}]$, 4) $[0.75 \mathrm{~mm} \mathrm{D}, 2 \mathrm{~mm} \mathrm{P}$, 5) [0.75mm D, 2.5mm P], 6) [0.75mm $\mathrm{D}, 3 \mathrm{~mm} \mathrm{P}]$ and 7) $[1 \mathrm{~mm} \mathrm{D}, 2.5 \mathrm{~mm} \mathrm{P}$. Finally a pedicle screw, 6.5 $\mathrm{mm}$ in diameter $40 \mathrm{~mm}$ in length $[0.75 \mathrm{~mm} \mathrm{D}, 2 \mathrm{~mm} \mathrm{P}]$ was inserted to a depth of $40 \mathrm{~mm}$.

Outcomes measured were 1). mean pullout strength, and 2). mode of failure in each testing group. Axial pullout testing was performed using an MTS 858 Mini Bionix test machine at a rate of $5 \mathrm{~mm} / \mathrm{min}$ on ten blocks from each group. The means were analyzed using ANOVA statistical analysis ( $\mathrm{p} \leq 0.05)$.

Results: In the axial pullout tests, the probed group had the highest average maximum load $761 \pm 25 \mathrm{~N}$. The other six groups' results were as follows: $[0.5 \mathrm{~mm} \mathrm{D}, 1.5 \mathrm{~mm} \mathrm{P}=713 \pm 53 \mathrm{~N}],[0.5 \mathrm{~mm} \mathrm{D}$, $2 \mathrm{~mm} \mathrm{P}=726 \pm 55 \mathrm{~N}],[0.75 \mathrm{~mm} \mathrm{D}, 2 \mathrm{~mm} \mathrm{P}=698 \pm 57 \mathrm{~N}],[0.75 \mathrm{~mm}$ $\mathrm{D}, 2.5 \mathrm{~mm} \mathrm{P}=758 \pm 48 \mathrm{~N}], 0.75 \mathrm{~mm} \mathrm{D}, 3 \mathrm{~mm} \mathrm{P}=755 \pm 48 \mathrm{~N}],[1 \mathrm{~mm}$ $\mathrm{D}, 2.5 \mathrm{~mm} \mathrm{P}=717 \pm 45 \mathrm{~N}]$. There was no significant difference between the groups. The mode of failure was screw pullout in all the groups except in group 1 (probe only) where $50 \%$ of the bone blocks fractured before the screw pulled out.

Conclusions: In an osteoporotic bone model, changing the pitch of the tap has no statistical effect on axial pullout or cantilever bend strength. Osteoporotic bone may render tap design features (pitch and depth) marginal and highlights the need for cement augmentation. The high rate of fracture in the probe group was a surprising result which may imply the potential benefit of tapping in order to prevent catastrophic failure of bone.

Paper \#33. 3D Molecular And Morphological Characterization Of The Intervertebral Disc Using Affinity- And ExclusionBased Contrast-Enhanced MicroCT

Tristan Maerz, MS; Michael D. Newton, BS; Shannon Timmons, PhD; Nathan Delaney, BS; Michael Pirrone, BS; Daniel K. Park, MD; Kevin C. Baker, PhD

\section{Beaumont Health}

Introduction: Our group previously demonstrated the ability to map intervertebral disc degeneration using micro-computed tomography $(\mu \mathrm{CT})$ with a contrast agent that is preferentially-excluded from sulfated-glycosaminoglycan (sGAG)-rich areas. This study aims to compare two iodine-containing contrast agents: a negatively charged agent preferentially excluded from sGAG-rich tissues (exclusion-based), and a positively-charged agent attracted (affinitybased) to sGAG-rich tissues.

Methods: The exclusion-based contrast agent (EB) was ioxaglate. The affinity-based contrast agent (AB), CA4+, was synthesized using a previously described protocol. Lumbar IVDs were dissected from Lewis rats. Based on optimization, 24-hour contrast incubation in $40 \% \mathrm{v} / \mathrm{v} \mathrm{EB}$ and $20 \mathrm{~g} \mathrm{I} / \mathrm{L} \mathrm{AB}$ were performed prior to $\mu \mathrm{CT}$ imaging. Following optimization, whole IVDs were digested for 24 hours in $50 \mathrm{U} / \mathrm{mL}$ papain to deplete sGAG content within the IVD simulating degeneration. Contrast-enhanced $\mu \mathrm{CT}$ imaging was performed pre- and post-digestion. 3D IVD volumes were isolated, and mean attenuations and 2D disc height maps were calculated. Biochemical assays and histology (Safranin-O staining) were used to correlate $\mu \mathrm{CT}$ attenuation to SGAG content for multiple digestion time points.

Results: Both $\mathrm{EB}$ and $\mathrm{AB}$ produced marked IVD contrast enhancement (Fig-1). The sGAG-rich NP exhibited lower attenuation than the AF using $\mathrm{EB}$ and higher attenuation using $\mathrm{AB}$, consistent with the exclusion-based and affinity-based mechanisms of each agent. Post-digestion, IVD attenuation significantly increased when imaged with $\mathrm{EB}(11.51 \% \pm 1.84, \mathrm{P}=0.053)$, and decreased using $\mathrm{AB}$ $(-8.76 \% \pm 0.32, \mathrm{P}=0.020)$. Normalized by iodine concentrations, $\mathrm{AB}$ was significantly more sensitive to sGAG depletion in the IVD than $\mathrm{EB}$ (EB: $0.09 \% / \mathrm{gI} \pm 0.01 ; \mathrm{AB}:-0.44 \% / \mathrm{gI} \pm 0.02, \mathrm{P}=0.002$ ). Contrast enhancement using both $\mathrm{EB}$ and $\mathrm{AB}$ also enabled morphological evaluation (Fig-2). 

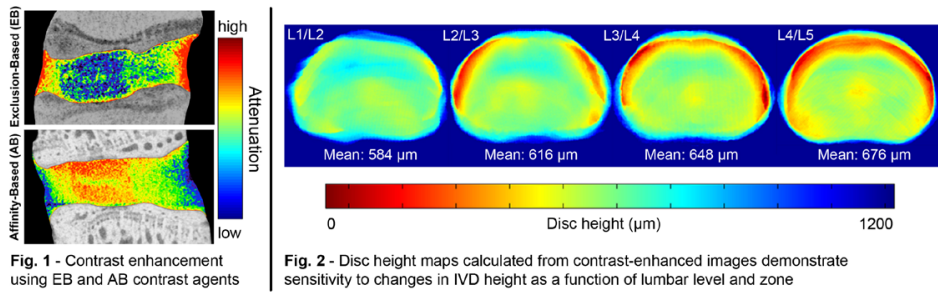

Conclusions: Contrast enhancement of the rat IVD is possible using both exclusion-based and affinity-based iodinated agents. Both agents demonstrate sensitivity to spatial and temporal differences in sGAG content and enable accurate 3D segmentation for morphological characterization. Due to the greater formal charge ( +4 for $\mathrm{AB},-1$ for $\mathrm{EB}), \mathrm{AB}$ provides greater sensitivity to differences in sGAG content per gram iodine, indicating that $\mathrm{AB}$ may be sensitive to smaller changes in sGAG content. Both agents can be used effectively to image the IVD in preclinical models of degeneration or regeneration.

Paper \#34. Differing Cytokine Profile in Intervertebral discs Associated with and without Modic Changes

Gregory D. Schroeder, MD; Dessislava Markova, PhD; John Koerner, MD; Alexander R. Vaccaro, MD, PhD; Christopher K. Kepler, MD, MBA; David Greg Anderson, $M D$

The Rothman Institute at Thomas Jefferson University

Introduction: Recent literature suggests an anaerobic infection with a low virulence organism such as P. acnes may be the cause of Modic changes. Anaerobic bacteria frequently enter the blood stream after tooth brushing and neovascularization after a herniated disc may provide a pathway for anaerobic bacteria to colonize the avascular nucleus pulposus. If Modic changes are due to an infectious cause rather than a degenerative one, the cytokine profile of the intervertebral disc should be significantly different in discs with an without Modic changes. The aim of this study is to compare the cytokine profile in degenerative intervertebral discs with and without Modic changes.

Methods: Patients undergoing an anterior lumbar interbody fusion were matched based on the Pfirrmann grade and the presence or absence of Modic changes. The intervertebral disc tissue was collected during the surgery and analyzed for cytokine expression using a protein antibody array (RayBio Human Cytokine Antibody Array 3). A Student's T-test was used to compare cytokine expression between discs without Modic changes and those with Type 2 Modic change. An ELISA was performed to verify the results.

Results: The cytokine profile of six intervertebral discs, three associated with type two Modic changes and three without Modic

\begin{tabular}{|l|c|c|c|c|}
\hline \multicolumn{7}{|c|}{ Table 1 } \\
\hline GM-CSF & Modic 0 & Modic 2 & Fold increase & P value \\
\hline CXCL5 & 45.5 & 335.2 & 7.37 & 0.001 \\
\hline MIP-1-delta & 208.4 & 744.6 & 3.57 & 0.04 \\
\hline TNF-beta & 439.3 & 907.2 & 2.07 & 0.09 \\
\hline GRO-alpha & 294.1 & 1070.0 & 3.64 & 0.09 \\
\hline IL-1beta & 183.8 & 359.5 & 4.80 & 0.10 \\
\hline TNF-alpha & 328.1 & 979.3 & 3.16 & 0.12 \\
\hline IL-8 & 642.4 & 1517.3 & 3.04 & 0.22 \\
\hline IL-6 & 108.0 & 170.5 & 1.58 & 0.27 \\
\hline RANTES & 3054.0 & 3107.5 & 1.02 & 0.46 \\
\hline
\end{tabular}

GM CSF indicates granulocyte-macrophage colony-stimulating factor; MIP indicates macrophage inflammatory protein; TNF indicates tumor necrosis factor; IL indicates interleukin changes, was analyzed and the relevant results are presented in Table 1. There was a seven-fold increase in the level of granulocyte-macrophage colony-stimulating factor (GM-CSF) (p $=0.001)$ and a three-and-a-half-fold increase in the level of CXCL5 ( $\mathrm{p}=0.04)$.

Discussion: The major function of GM-CSF is to stimulate differentiation, proliferation and activation of macrophages and dendritic cells, which are required to activate a T-cell mediated immune response. Similarly, CXCL5 is a potent neutrophil chemotactic cytokine. Additionally, the results question the correlation between Modic changes and painful intervertebral discs, as the expression of RANTES, a cytokine that has been shown to be significantly increased in painful discs was almost identical $(\mathrm{p}=0.92)$ in discs with and without Modic changes.

Conclusion: The cytokine profile in intervertebral discs associated with and without Modic changes is significantly. The presence of an increase in MG-CSF and CXCL5 further validates the possible infectious cause.

\section{Paper \#35. Effect of rhBMP-2 on Lung Cancer Spine Metastasis in Rodents}

Abhishek S. Kannan, BS; Kevin Sonn, MD; Sharath Bellary, MD; Chawon Yun, PhD; Sohaib Hashmi, MD; John Nelson, MD; Amruta Ashtekar, PhD; Anjan Ghosh; Michael Nickoli, MD; Jason Ghodasra, MD; Stuart Stock, PhD; Erin L. Hsu, PhD; Wellington $\mathrm{Hsu}, \mathrm{MD}$

\section{Northwestern Feinberg School of Medicine}

Introduction: Lung cancer is the second most prevalent cancer, and spinal metastases are found in $30-90 \%$ of patients with death attributed to cancer. Due to bony destruction caused by metastases, surgery is often required to restore spinal alignment and stability. The use of rhBMP-2 in patients with a history of cancer is not widely recommended due to the controversial question of its involvement in tumor propagation. Current literature provides no consensus on the effects of BMP on metastasis. Direct intraosseous injection of cancer cells into the vertebral body affords the opportunity to quantify the effects of rhBMP-2 on individual metastatic spine lesions without interference from other metastases..

Methods: Luciferase-labeled A549 human lung adenocarcinoma cells were pre-treated with saline (control) or $100 \mathrm{ng} / \mathrm{ml} \mathrm{rhBMP-2}$ (BMP), followed by implantation into the L5 vertebral bodies (VB) of 42 athymic rats ( $5 \times 104$ cells/rat). After 4 weeks, in vivo bioluminescent imaging (BLI) was performed, and average signal radiance was quantitated. Radiography, microCT, and histological analyses were used to visualize and quantitate the impact of rhBMP-2 pretreatment on tumor -induced osteolysis.

Results: Four weeks post-implantation, BLI showed focal signals in the L5 VB of 13/14 control animals (93\%) and 16/18 from the BMP treatment group (89\%). Average tumor burden was $7.43 \times 103$ $\mathrm{p} / \mathrm{s} / \mathrm{cm} 2 / \mathrm{sr}$ and $1.11 \times 104 \mathrm{p} / \mathrm{s} / \mathrm{cm} 2 / \mathrm{sr}$ for the control and BMP groups, respectively (Fig 1a). Radiographic and microCT imaging

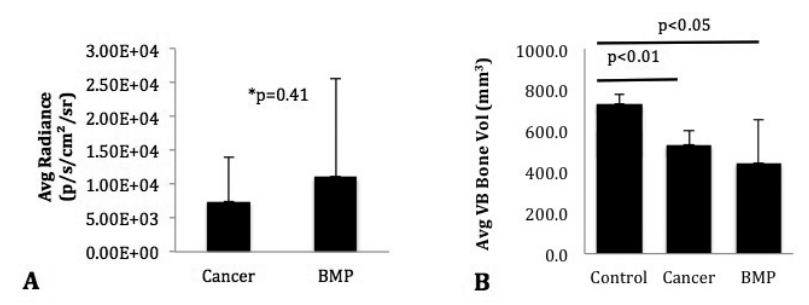

Fig 1. (a) Average radiance measurement from bioluminescent imaging (BLI) demonstrating no difference between treatment groups. (b) MicroCT quantification of vertebral body bone mass demonstrating significant bone loss in both groups compared to age matched controls but no difference between groups. 
demonstrated osteolysis in $100 \%$ of animals with positive BLI focal signals. Significant bone loss was found in both control and BMP groups compared to age-matched untreated controls, but there were no differences average radiance, size of the osteolytic lesion, or radiographic appearance between the study groups (Fig 1b).

Conclusion: We established a reliable in vivo model to study isolated spinal metastases from lung cancer. Our results demonstrate that in vitro exposure of lung cancer cells to rhBMP-2 does not promote the growth and development of spinal lesions in the rat. Subsequent studies will employ a more clinically relevant approach, with evaluation of the effects of rhBMP-2 application in a posterolateral fusion setting after establishment of both primary lung and secondary spinal metastatic lesions.

Paper \#36. Primary Versus Revision Single-Level Minimally Invasive Lumbar Discectomy: Analysis of Clinical Outcomes and Narcotic Utilization

Junyoung Ahn, BS; Ehsan Tabaraee, MD; Daniel D. Bohl, MPH; Khaled Aboushaala, MD; Kern Singh, MD

\section{Rush University Medical Center}

Introduction: Revision spine surgery may be associated with longer procedural time and greater soft tissue disruption. Few studies have analyzed the surgical outcomes and narcotic utilization associated with minimally invasive (MIS) revision lumbar discectomies. As such, the purpose of the present study was to compare the intra-operative variables, surgical outcomes, and narcotic utilization between primary and revision 1-level MIS lumbar discectomies.

Methods: A retrospective analysis of 227 prospectively followed patients who underwent an MIS 1-level lumbar discectomy for degenerative spinal pathology between 2009-2014 by a single sur-

Table 1: Surgical Outcomes for Primary and Revision 1-Level MIS Lumbar Discectomy

\begin{tabular}{|c|c|c|c|}
\hline & Primary & Revision & p-value \\
\hline Procedural time (min) & $36.1 \pm 16.8$ & $42.4 \pm 14.5$ & $<0.05$ \\
\hline EBL $(c c) \dagger$ & $41.1 \pm 23.3$ & $43.7 \pm 21.8$ & 0.50 \\
\hline LOS (Hours) $\ddagger$ & $13.1 \pm 13.8$ & $18.6 \pm 17.4$ & $<0.05$ \\
\hline \multicolumn{4}{|l|}{$\underline{\text { VAS Score * }}$} \\
\hline 6-Week & $2.5 \pm 2.5$ & $3.3 \pm 2.9$ & 0.10 \\
\hline 12-Week & $2.9 \pm 3.0$ & $2.9 \pm 3.0$ & 0.90 \\
\hline 6-Month & $2.5 \pm 3.2$ & $3.0 \pm 3.5$ & 0.70 \\
\hline \multicolumn{4}{|l|}{ Complication Rate } \\
\hline Intra-operative§ & $2(1.1 \%)$ & 0 & 0.50 \\
\hline In-Hospital & 0 & 0 & - \\
\hline 6 week & 0 & 0 & - \\
\hline 12 week $\dagger \dagger$ & $1(0.5 \%)$ & 0 & 0.60 \\
\hline 6 month $¥ \ddagger$ & 0 & $1(2.4 \%)$ & $<0.05$ \\
\hline Reherniation Rate & $13(7.0 \%)$ & $6(14.6 \%)$ & 0.09 \\
\hline Re-operation Rate & $27(14.5 \%)$ & $13(31.7 \%)$ & $<0.05$ \\
\hline \multicolumn{4}{|l|}{ Type of Re-operation } \\
\hline Lumbar decompression & $16(59.3 \%)$ & $1(7.7 \%)$ & 0.30 \\
\hline Lumbar fusion & $11(40.7 \%)$ & $12(92.3 \%)$ & $<0.05$ \\
\hline Incision and drainage & 0 & 0 & - \\
\hline Dural repair & 0 & 0 & - \\
\hline \multicolumn{4}{|l|}{ Time to Re-operation ** } \\
\hline Lumbar decompression & $176.1(11-1253)$ & 35.0 & \\
\hline Lumbar fusion & $222.6(102-494)$ & $378.1(124-661)$ & \\
\hline Incision and drainage & 0 & 0 & \\
\hline Dural repair & 0 & 0 & \\
\hline \multicolumn{4}{|l|}{$\begin{array}{l}\text { \$ - Incidental durotomy } \\
+\downarrow \text { - 1 Iatrogenic spondylolisthesis } \\
¥ \neq \text { - 1 Iatrogenic spondylolisthesis } \\
\star \star \star \\
\text { - Mean time (range) in days after }\end{array}$} \\
\hline
\end{tabular}

geon was performed. Patients were stratified into primary and revision cohorts. Demographics, comorbidity, intra-operative parameters, peri- and post-operative outcomes, and re-operations were assessed. Post-operative narcotic utilization was compared between cohorts. Statistical analyses were performed utilizing Student's T-test and Pearson's chi-squared test. A p-value $<0.05$ denoted statistical significance.

Results: Of the 227 patients included, 41 (18.1\%) underwent revision surgery. Demographics, comorbidity, smoking status, preoperative visual analogue scale (VAS) scores, and estimated blood loss (EBL) did not differ between cohorts. However, the revision cohort demonstrated a longer procedural time, increased length of hospitalization, and higher post-operative narcotic utilization (Table 1). Although not statistically significant, revision patients trended towards higher 6-week post-operative VAS scores and re-herniation rates. In addition, revision patients were more likely to undergo a subsequent lumbar fusion than primary patients.

Discussion: The findings suggest that revision MIS lumbar discectomy may be associated with increased procedural time, increased length of hospitalization, and increased post-operative narcotic utilization. While revision patients trended towards higher post-operative VAS scores at 6-weeks, both cohorts demonstrated similar pain levels at final follow-up. Lastly, revision patients may be at a greater risk of re-herniation and subsequent re-operation.

Conclusion: These differences between primary and revision procedures should be thoroughly discussed with patients during preoperative counseling for revision procedures.

Paper \#37. Thromboembolic Events After Traumatic Lumbar Vertebral Fractures: An Analysis Of 80,558 Patients

Andre M. Samuel, BBA; Matthew L. Webb, AB; Adam M. Lukasiewicz, MSc; Bryce A. Basques, BS; Daniel D. Bohl, MPH; Jonathan Newman Grauer, MD

Yale School of Medicine

Introduction: Thromboembolic events are a significant source of potentially preventable morbidity and mortality in trauma patients, even with widespread use of prophylaxis. In patients with traumatic lumbar vertebral fractures, a common high-energy injury often resulting in neurological deficit, it remains to be seen what factors may be associated with thromboembolic events.

Methods: All patients with lumbar vertebral fractures in the American College of Surgeons National Trauma Data Bank Research Data Set (NTDB RDS) from years 2011 and 2012 were identified. Multivariate logistic regression was used to determine factors associated with occurrence of either deep vein thrombosis or pulmonary embolism. Factors considered included age, associated injuries, Injury Severity Score, and adverse events.

Results: A total of 80,558 patients with lumbar vertebral fractures were included in the 2011 and 2012 NTDB. The overall rate of thromboembolic events was $2.8 \%$.

The rate of thromboembolic events increased to $15 \%$ or greater in certain subsets of the population (Table 1): patients with decubitus ulcers (19.5\%), patients with surgical site infections $(18.1 \%)$, patients with unplanned returns to the operating room $(16.4 \%)$, patients with pneumonia (15.6\%), and patients with admissions longer than 21 days $(15.0 \%)$.

In multivariate analysis (Table 1), several of these factors were still found to be associated with occurrence of thromboembolic events, even after controlling for known risk factors such as age, injury severity, length of stay, and immobilization. As expected age, injury severity, length of stay, and advanced hospital care were all highly associated with increased thromboembolic events.

Discussion: While the overall rate of thromboembolic events is low after lumbar vertebral fractures, risk factors such as decubitus 
ulcers, surgical site infections, and pneumonia were associated with increased occurrence of events, even after controlling for age, length of stay, and injury severity, with rates of up to $19.5 \%$. While causation cannot be implied due to the lack of temporal data regarding thromboembolic events in NTDB, the increased rates of events in certain subpopulations are striking and suggest need for more aggressive prophylaxis strategies in these subsets of patients.

Conclusion: More aggressive thromboembolism prophylaxis is needed in certain patients with risk factors for thromboembolic events.

\section{Paper \#38. The Economic Impact of an Incidental Durotomy}

Gregory D. Schroeder, MD; Christopher K. Kepler, MD, MBA, Pouya Alijanipour, MD; Priyanka R. Kumar, BA; Mark F. Kurd, $M D$; Kris Radcliff, MD; Jeffrey Rihn, MD; Alan S. Hilibrand, MD; Alexander R. Vaccaro, $M D, P h D$

The Rothman Institute at Thomas Jefferson University

Introduction: An incidental durotomy during lumbar spine surgery does not affect long-term outcomes, but as reimbursement moves towards bundle payments, it may substantially affect the profitability of spine surgery. The purpose of this study is to determine the economic impact of an incidental durotomy.

Methods: Retrospectively reviewing a prospectively collected morbidity and mortality database, all patients with an incidental durotomy between $1 / 1 / 2012$ and 11/1/2013 were identified. Subjects were matched to controls (1:2) without a dural tear, and the total hospital charges for one year were collected. Controls were required to meet the following criteria: Age +/- 5 years; Charlson Comorbidity Index $(\mathrm{CCI})+/-1$; Date of surgery +/- 2 years; Exact region of the spine, but not the exact level (i.e. lumbar $\rightarrow$ lumbar); Exact type of fusion (i.e. approach, instrumentation); Exact number of levels fused; Use of rhBMP-2; Number of levels decompressed $+/-1$. All hospital charges for the first 90 days after surgery were

\begin{tabular}{|c|c|c|c|}
\hline \multicolumn{4}{|c|}{ Table 1} \\
\hline & Dural Tear & No Dural Tear & $P$ Value \\
\hline Total number of patients & 60 & 120 & \\
\hline Average age in years & 61.0 & 60.9 & 0.4 \\
\hline Average charlson comorbidity Index & 0.86 & 0.79 & 0.83 \\
\hline Number of cervical cases & 3 & 6 & 1.00 \\
\hline Number of thoracic cases & 0 & 0 & 1.00 \\
\hline Number of lumbar cases & 57 & 114 & 1.00 \\
\hline Total levels decompressed & 128 & 232 & 0.23 \\
\hline Total levels fused & 40 & 80 & 1.00 \\
\hline Total interbody fusions & 17 & 34 & 1.00 \\
\hline Average length of stay in days & 3.7 & 2.8 & $0.02^{*}$ \\
\hline $\begin{array}{l}\text { Average time in the operating room In } \\
\text { minutes }\end{array}$ & 253.2 & 222.4 & $0.02^{*}$ \\
\hline $\begin{array}{l}\text { Hospital charges per patient for the initial } \\
\text { surgical admission }\end{array}$ & $\$ 92,221.06$ & $\$ 77,506.61$ & $0.04^{*}$ \\
\hline Total hospital charges per patient for 30 days & $\$ 92,610.14$ & $\$ 79,294.75$ & $0.04^{*}$ \\
\hline Total hospital charges per patient for 90 days & $\$ 92,945.31$ & $\$ 81,997.74$ & 0.1 \\
\hline Total hospital charges per patient for 1 year & $\$ 96,994.62$ & $\$ 81,997.74$ & $0.03^{*}$ \\
\hline $\begin{array}{l}\text { Hospital charges per patient from discharge } \\
\text { through } 30 \text { days }\end{array}$ & $\$ 389.08$ & $\$ 1,788.14$ & 0.38 \\
\hline $\begin{array}{l}\text { Hospital charges per patient from discharge } \\
\text { through } 90 \text { days }\end{array}$ & $\$ 724.25$ & $\$ 4,491.14$ & 0.18 \\
\hline $\begin{array}{l}\text { Hospital charges per patient from discharge } \\
\text { through } 1 \text { year }\end{array}$ & $\$ 4,773.57$ & $\$ 4,491.14$ & 0.67 \\
\hline Number of patients who were readmitted & $3(5 \%)$ & $6(5 \%)$ & 1.00 \\
\hline $\begin{array}{l}\text { Number of patients who returned to ER } \\
\text { without readmissions }\end{array}$ & $5(8.3 \%)$ & $7(5.8 \%)$ & 0.56 \\
\hline $\begin{array}{l}\text { Number of patients who required an } \\
\text { additional operation }\end{array}$ & $4(6.7 \%)$ & $7(5.8 \%)$ & 0.99 \\
\hline *Statistically & nificant & & \\
\hline
\end{tabular}

collected, and after 90 days only charges directly related to spine care were collected.

Results: Of the 136 patients identified with an incidental durotomy, two controls could be identified for 60 patients. No differences between the groups were identified (Table 1). A statistically significant increase in the total charges for patients with an incidental durotomy was identified at the initial hospital visit $(\$ 92,221.06$ versus $\$ 77,506.61 \mathrm{p}=0.04)$, the total charges for 30 days $(\$ 92,610$ versus $\$ 79,295, \mathrm{p}=0.04)$ and the total charges for the year $(\$ 96,995$ versus $\$ 81,998, \mathrm{p}=0.03)$. However, the incremental hospital charges between discharge and thirty days $(\$ 389$ versus $\$ 1,788, \mathrm{p}=$ $0.40), 90$ days $(\$ 724$ versus $\$ 4,491, \mathrm{p}=0.18)$ and one year $(\$ 4,774$ versus $\$ 4,491, \mathrm{p}=0.67$ ) were not significant (Table 1 ).

Conclusion: An incidental durotomy statistically increases the initial hospital charges for patients undergoing spine surgery, however it has no effect on hospital charges after discharge. In a bundled payment system this may create a disincentive for surgeons to treat patients with conditions such as facet cysts or recurrent stenosis that significantly increase the rate of dural tears.

Paper \#39. Oblique Magnetic Resonance Imaging of the Lumbar Neuroforamina Improves Precision When Diagnosing Nerve Root Impingement: A Prospective Study

David B. Bumpass, MD; Jacob M. Buchowski, MD, MS; Michael P. Kelly, MD; Michelle Miller-Thomas, MD

\section{Washington University}

Introduction: Evaluation of lumbar foraminal stenosis (LFS) using magnetic resonance imaging (MRI) is subject to variability of interpretation. The lumbar nerve roots exit the foramina oblique to all three standard imaging planes, potentially contributing to this imprecision. Because inadequate decompression is the most common cause of failed lumbar surgery, improving the precision of LFS diagnosis would potentially provide surgeons more clarity in operative planning. We developed an oblique T1 fast spin-echo MRI sequence providing high-resolution imaging of the lumbar roots in the plane of the neuroforamina (Figure 1). We hypothesized that this oblique MRI technique would improve both inter- and intra-rater reliability for grading LFS.

Methods: Twenty-two patients with radicular symptoms prospectively underwent both standard non-contrast lumbar MRI and the experimental oblique MRI using a $1.5 \mathrm{~T}$ scanner. Patients with previous lumbar surgery, lumbar scoliosis $>20^{\circ}$, or inability to undergo MRI were excluded.

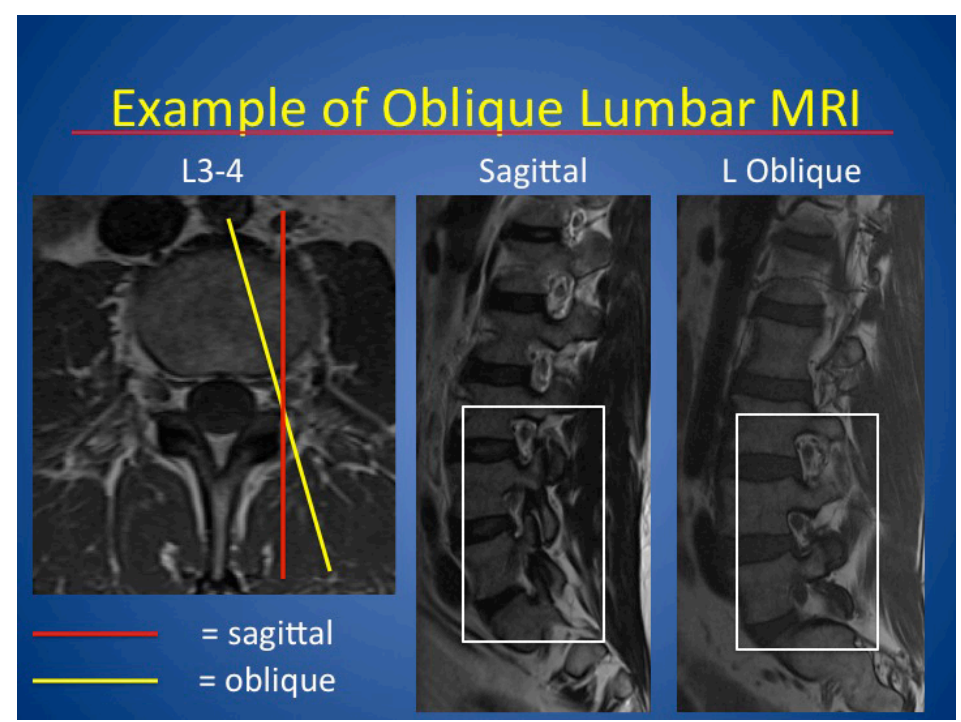


Three spine surgeons and a neuroradiologist graded the severity of foraminal stenosis for each patient using a validated 4-point scale. LFS was graded using both the standard T1 sagittal and the oblique images; grading was then repeated 4 weeks later. Intra-class correlation coefficients (ICC) were used to calculate inter- and intrarater reliability; significance was defined as non-overlapping $95 \%$ confidence intervals (CI). Reviewers were asked "yes/no" for each patient if the oblique series provided additional clarity/diagnostic confidence over the standard sagittal series.

Results: Inter-rater agreement was significantly better for the oblique sequences, with an ICC of 0.75 (95\% CI 0.71-0.78) as compared to an ICC of 0.64 for the standard sagittal sequences $(95 \%$ CI 0.55-0.71). Similarly, intra-rater agreement for the oblique scans demonstrated significant superiority, with an ICC of 0.84 (95\% CI $0.82-0.86$ ); the ICC for the sagittal scans was 0.79 (95\% CI 0.77 0.82 ). The number of "yes" responses per patient displayed strong and significant correlation with the mean stenosis grade for each patient (rho $=0.63, \mathrm{p}=0.02$ ).

Discussion and Conclusions: In a prospective study, a novel oblique lumbar MRI sequence improved diagnostic consistency when evaluating LFS in patients with radiculopathy, as compared to standard sagittal MRI. The oblique sequence was of particular value in patients with multilevel degenerative changes.

Paper \#40. Neuroforaminal Bone Growth Following Minimally Invasive Transforaminal Lumbar Interbody Fusion with BMP: A Computed Tomographic Analysis

Junyoung Ahn, BS; Anton Y. Jorgensen, MD; Khaled Aboushaala, $M D ;$ Kern Singh, $M D$

Rush University Medical Center

Introduction: BMP induces osteoblast differentiation leading to new bone formation. The objective of this study is to identify radiographic patterns of symptomatic neuroforaminal bone growth in patients who have undergone a single-level minimally invasive transforaminal lumbar interbody fusion (MIS-TLIF) augmented with bone morphogenetic protein (BMP) utilizing computed tomography (CT).

\begin{tabular}{|c|c|c|c|}
\hline & Symptomatic & Asymptomatic & p-value \\
\hline Count (n) & 18 & 13 & \\
\hline$\%$ Female & $11.1 \%$ & $38.5 \%$ & 0.07 \\
\hline Age (years) & $41.4 \pm 8.9$ & $47.6 \pm 10.0$ & 0.08 \\
\hline BMP (mg) & $4.8 \pm 2.7$ & $5.1 \pm 3.2$ & 0.82 \\
\hline $\begin{array}{c}\text { BMP Kits (n) } \\
\text { X-small }(2.1 \mathrm{mg}) \\
\text { Small }(4.2 \mathrm{mg}) \\
\text { Large }(12.0 \mathrm{mg})\end{array}$ & $\begin{array}{c}11.1 \%(\mathrm{n}=2) \\
77.8 \%(\mathrm{n}=14) \\
11.1 \%(\mathrm{n}=2)\end{array}$ & $\begin{array}{l}15.4 \%(\mathrm{n}=2) \\
69.2 \%(\mathrm{n}=9) \\
15.4 \%(\mathrm{n}=2)\end{array}$ & 0.87 \\
\hline $\begin{array}{l}\text { Levels (n) } \\
\text { L3-L4 } \\
\text { L4-L5 } \\
\text { L5-S1 }\end{array}$ & $\begin{array}{c}5.6 \%(\mathrm{n}=1) \\
16.7 \%(\mathrm{n}=3) \\
77.8 \%(\mathrm{n}=14)\end{array}$ & $\begin{array}{l}15.4 \%(n=2) \\
38.5 \%(n=5) \\
46.2 \%(n=6)\end{array}$ & 0.19 \\
\hline \multicolumn{4}{|c|}{ Mean Axial Section Bone Growth $\left(\mathrm{mm}^{2}\right)$} \\
\hline Foraminal level & $43.6 \pm 53.5$ & $56.7 \pm 36.5$ & 0.38 \\
\hline Disc level & $164.0 \pm 92.4$ & $77.0 \pm 104.9$ & $<0.05$ \\
\hline Lateral recess & $69.6 \pm 70.5$ & $5.9 \pm 12.5$ & $<0.01$ \\
\hline \multicolumn{4}{|c|}{ Mean Sagittal Section Bone Growth $\left(\mathrm{mm}^{2}\right)$} \\
\hline Foraminal & $123.4 \pm 116.2$ & $41.8 \pm 51.6$ & $<0.05$ \\
\hline Sub-articular & $148.7 \pm 185.1$ & $35.8 \pm 37.4$ & $<0.05$ \\
\hline Extra-foraminal & $26.4 \pm 72.2$ & $8.1 \pm 19.1$ & 0.40 \\
\hline \multicolumn{4}{|c|}{$\underline{\text { Total Axial and Sagittal Section Bone Growth }\left(\mathrm{mm}^{2}\right)}$} \\
\hline Total Axial Area & $290.3 \pm 162.1$ & $119.4 \pm 115.6$ & $<0.01$ \\
\hline Total Sagittal Area & $298.4 \pm 324.4$ & $85.8 \pm 76.3$ & $<0.05$ \\
\hline
\end{tabular}

Methods: A radiographic analysis of 31 patients who underwent an MIS-TLIF with an intervertebral cage and BMP performed by a single surgeon between the years of 2008 and 2014 was performed. Medical records were reviewed to identify patients who developed recalcitrant post-operative radiculopathy. Asymptomatic patients who had underwent CT imaging for other reasons were identified. Post-operative CT of the symptomatic and asymptomatic patients were analyzed to identify patterns of neuroforaminal bone growth on axial and sagittal sections. Independent sample t-test was utilized to compare the area of neuroforaminal bone growth in symptomatic and asymptomatic patients.

Results: Post-operative CT images of 18 symptomatic patients were compared to those of 13 asymptomatic patients. Mean areas

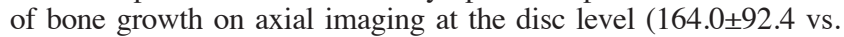
$77.0 \pm 104.9 \mathrm{~mm} 2)$, lateral recess $(69.6 \pm 70.5$ vs. $5.9 \pm 12.5 \mathrm{~mm} 2)$ as well as total cross-sectional area $(290.3 \pm 162.1$ vs. $119.4 \pm 115.6$ $\mathrm{mm} 2)$ were greater in symptomatic patients $(\mathrm{p}<0.05)$. On sagittal imaging, the mean bone growth at the foraminal level $(123.4 \pm 116.2$ vs. $41.8 \pm 51.6 \mathrm{~mm} 2)$, at the sub-articular level $(148.7 \pm 185.1 \mathrm{vs}$. $35.8 \pm 37.4 \mathrm{~mm} 2)$ and the total bone growth $(298.4 \pm 324.4$ vs. $85.8 \pm 76.3 \mathrm{~mm} 2)$ were greater in symptomatic patients $(\mathrm{p}<0.05)$. The mean amount of BMP utilized was similar between symptomatic and asymptomatic patients ( $4.8 \pm 2.7$ vs. $5.1 \pm 3.2 \mathrm{mg}$, respectively).

Discussion:Extension of bone growth toward the traversing nerve root was associated with recalcitrant post-operative radiculopathy following MIS-TLIF with BMP. Significantly greater bone growth was demonstrated at the level of the disc, the lateral recess, foraminal level, and sub-articular level in symptomatic patients on CT imaging.

Conclusions: The authors suggest closely evaluating the radiographic evidence of bone growth at the lateral recess in patients with recalcitrant radiculopathy following BMP utilization.

Paper \#41. Surgeon Specialty Influences Referral Rate for Osteoporosis Management Following Vertebral Compression Fractures

Scott D. Daffner, MD; Jonathan M. Karnes, MD; Colleen M. Watkins, $M D$

West Virginia University

Introduction: Fragility fractures are not isolated events, but symptoms of an underlying disease process. Although initiatives such as "Own the Bone" have targeted orthopaedic surgeons to raise awareness of the need for osteoporosis management in patients presenting with fragility fractures, vertebral compression fractures are unique as they are also frequently managed by physicians of other

\section{Referral Rate for Osteoporosis Management}

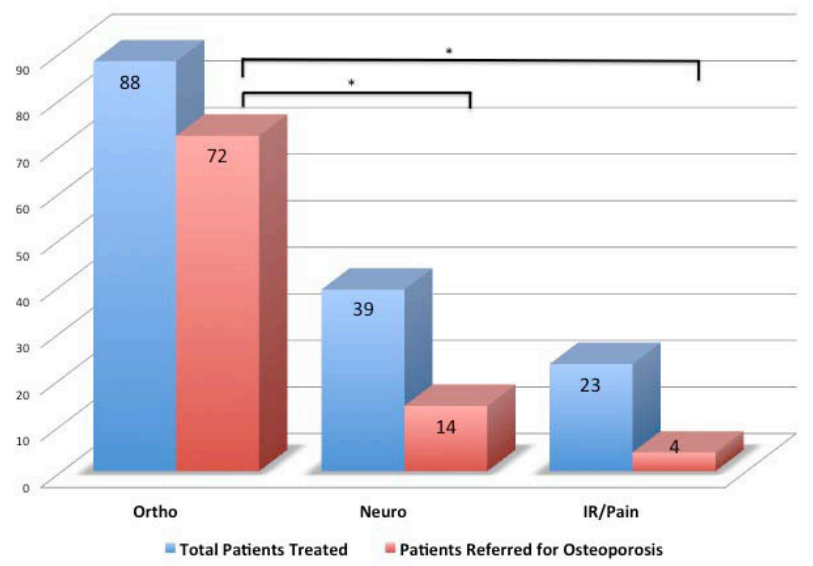


specialties, including neurosurgery, pain management, and interventional radiology who are not the target of international osteoporosis awareness programs and do not surgically manage other osteoporosis-associated fractures. The purpose of this study was to evaluate the referral rate for long-term osteoporosis management following vertebral compression fracture among patients treated by different specialties at a single academic institution.

Methods: A retrospective chart review identified patients at a single academic hospital undergoing vertebroplasty or kyphoplasty for painful osteoporotic compression fractures from March 2009 to March 2014. Patients treated for fractures due to tumor or trauma or those already receiving medical management of osteoporosis at the time of injury were excluded. Medical records were reviewed to determine if the treating surgeon discussed and/or referred the patient for long-term osteoporosis management at any time from the initial visit through the 90-day postoperative global period. Results were statistically analyzed with Chi square test.

Results: A total of 214 patients underwent vertebral cement augmentation; 150 patients met inclusion criteria ( $76 \%$ female, average age 72 years). Orthopaedic surgeons treated 88 patients, neurosurgeons treated 39 , and 23 were treated by interventional radiology or pain management physicians. The overall referral rate for osteoporosis management was $60 \%(90 / 150)$. Orthopaedic surgeons had a referral rate of $82 \%(72 / 88)$, Neurosurgeons had a referral rate of $36 \%$ (14/39), and Interventional Radiology/Pain Management physicians had a referral rate of $17 \%(4 / 23)$. The referral rate was significantly higher $(\mathrm{p}<0.0001)$ for Orthopaedic surgeons compared to either of the other two groups; there was no significant difference between Neurosurgery and Interventional Radiology/Pain Management.

Conclusion: The results of this study shed light on the disparity in how different specialties approach treatment of osteoporosis in patients with fractures painful enough to require surgery. Moreover, the results highlight potential areas for improvement in osteoporosis awareness training.

Paper \#42. Percutaneous Pedicle Screw Fixation for Extension-type Fractures in Ankylosing Spondylitis and Diffuse Idiopathic Skeletal Hyperostosis

Cara L. Sedney, MD; Scott D. Daffner, MD; Abimbola ObafemiAfolabi, M.D.; Sanford E. Emery, MD, MBA; Steven C. Ludwig, M.D.; John C. France, M.D.

\section{West Virginia University}

Introduction: The use of percutaneous pedicle screw fixation is increasing due to the purported benefits of diminished blood loss, shorter operative time, and shorter recovery time. Patients with ankylosing spondylitis (AS) and diffuse idiopathic skeletal hyperostosis (DISH) are prone to extension-type fractures, but also have a propensity to form bone. Percutaneous pedicle screw fixation and direct osteosynthesis of these injuries may be an ideal approach for treating this subset of patients. The purpose of this study was to compare operative risks and surgical outcomes of patients with AS or DISH treated with percutaneous pedicle screw fixation and those treated with open posterior spinal fusion.

Methods: A retrospective review of patients treated for AS or DISH-associated thoracic or lumbar fractures at two academic, Level-1 trauma centers identified patients treated with either percutaneous pedicle screw fixation (MIS), open posterior fusion, or a combined "hybrid" technique. Records were reviewed for demographics, injury mechanism, surgical levels, operative time, blood loss, need for transfusion, length of stay, mortality, and maintenance of correction and fusion at follow-up.

Results: 37 patients were identified (19 MIS, 14 open, and 4 hybrid). Average follow-up was 18.2 months. Demographics were similar between groups. Four patients had noncontiguous injuries.
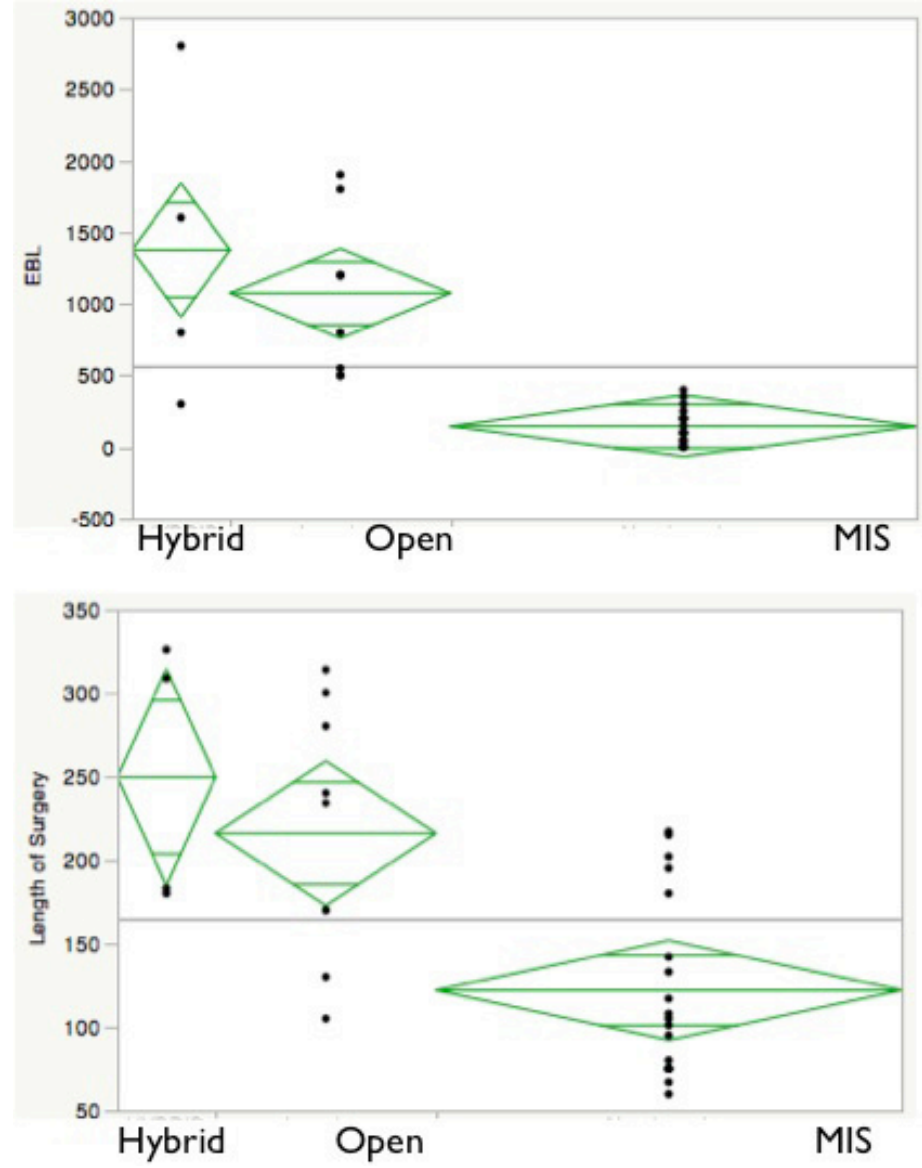

Average number of levels operated on was 5.2. Five patients died during initial hospitalization or during follow up. Three patients required washout due to wound infection ( 2 open, 1 hybrid). One patient (open) had hardware removal due to prominence after healing. The MIS group had significantly shorter operative time $(\mathrm{p}=0.0003)$, less blood loss $(\mathrm{p}<0.0001)$, and lower likelihood of transfusion $(\mathrm{p}=0.0014)$ than either the open or hybrid groups. The hybrid and open groups were similar to each other in this regard. There was no significant difference between the three subgroups in overall hospital stay or mortality. None of the patients for whom follow-up data was available demonstrated hardware loosening or failure of the fracture to heal.

Conclusions: The use of percutaneous pedicle screw fixation for treatment of extension-type fractures in patients with AS or DISH appears to be effective, and it is associated with shorter operative time and decreased blood loss.

Paper \# 43. The Surgical Algorithm for the AOSpine Thoracolumbar Spine Injury Classification System.

Gregory D. Schroeder, MD; Alexander R. Vaccaro, MD, PhD; Christopher K. Kepler, MD, MBA; John Koerner, MD; Marcel Dvorak, MD; Luiz R. Vialle, MD; Michael Fehlings, MD; Klaus Schnake, MD; Maximilian Reinhold, Priv.-Doz. Dr.; Jens R. Chapman, MD; S. RAJASEKARAN, MS(ORTH), MPhil, PhD; Frank Kandziora, $M D, P h D ; F C$ Oner, $M D, P h D$

The Rothman Institute at Thomas Jefferson University

Introduction: The AOSpine Thoracolumbar Spine Injury Classification System was published in 2013 and was designed to incorporate critical elements of existing classification systems. The 


\begin{tabular}{|c|c|c|c|c|c|c|c|c|c|}
\hline \multicolumn{10}{|c|}{ Table 1} \\
\hline & Europe (\%) & Asia (\%) & $\begin{array}{c}\text { South } \\
\text { America (\%) }\end{array}$ & $\begin{array}{c}\text { Middle East } \\
(\%)\end{array}$ & $\begin{array}{c}\text { North } \\
\text { America (\%) }\end{array}$ & Africa (\%) & $\begin{array}{c}\text { Glabsl } \\
(\%)\end{array}$ & $P$ Value & $\begin{array}{c}\mathrm{T} \\
\text { Aosts }\end{array}$ \\
\hline A2NO & $24(13.8)$ & $7(7.5)$ & $20(17.2)$ & $4(8.9)$ & $0(0.0)$ & $1(6.3)$ & $56(11.6)$ & $0.02+$ & 2 \\
\hline A3NOMO & $22(12.6)$ & $14(15.1)$ & $17(14.7)$ & $3(6.7)$ & $0(0.0)$ & $1(6.3)$ & $57(11.8)$ & 0.07 & 3 \\
\hline A3NOM1 & $117(67.2)$ & $57(61.3)$ & $77(66.4)$ & $31(68.9)$ & $16(41.0)$ & $11(68.8)$ & $\begin{array}{c}309 \\
(64.0)\end{array}$ & 0.06 & 4 \\
\hline A.3N1MO & $74(42.5)$ & $27(29.0)$ & $59(50.9)$ & $14(31.1)$ & $2(5.1)$ & $4(25.0)$ & $\begin{array}{c}180 \\
(37.3)\end{array}$ & $0.0001+$ & 4 \\
\hline A3N1M1 & $146(83.9)$ & $78(83.9)$ & $99(85.3)$ & $35(77.8)$ & $21(53.8)$ & $12(75.0)$ & $\begin{array}{c}391 \\
(81.0)\end{array}$ & $0.002+$ & 5 \\
\hline A3N2MO & $130(74.7)$ & $54(58.1)$ & $95(81.9)$ & $27(60.0)$ & $10(25.6)$ & $12(75.0)$ & $\begin{array}{c}328 \\
(67.9)\end{array}$ & $0.0001+$ & 5 \\
\hline $\mathrm{A} 3 \mathrm{~N} 2 \mathrm{M} 1$ & $154(88.5)$ & $79(84.9)$ & $113(97.4)$ & $40(88.9)$ & $31(79.5)$ & $14(87.5)$ & $\begin{array}{c}431 \\
(89.2) \\
\end{array}$ & $0.005+$ & 6 \\
\hline A4NOMO & $80(46.0)$ & $26(28.0)$ & $60(51.7)$ & $18(40.0)$ & $0(0.0)$ & $6(37.5)$ & $\begin{array}{c}190 \\
(39.3)\end{array}$ & $0.0001=+$ & 5 \\
\hline A4NOM1 & $142(81.6)$ & $60(64.5)$ & $95(81.9)$ & $36(80.0)$ & $19(48.7)$ & $7(43.8)$ & $\begin{array}{c}359 \\
(74.3) \\
\end{array}$ & $0.0001 *+$ & 6 \\
\hline A4N1MO & $108(62.1)$ & $56(60.2)$ & $81(69.8)$ & $27(60.0)$ & $7(17.9)$ & $10(62.5)$ & $\begin{array}{c}289 \\
(59.8) \\
\end{array}$ & $0.0001 \approx+$ & 6 \\
\hline$A 4 N_{1 M} 1$ & $144(82.8)$ & $72(77.4)$ & $104(89.7)$ & $37(82.2)$ & $23(59.0)$ & $14(87.5)$ & $\begin{array}{c}394 \\
(81.6)\end{array}$ & $0.002+$ & 7 \\
\hline$A 4 N 2 M O$ & $137(78.7)$ & $61(65.6)$ & $98(84.5)$ & $33(73.3)$ & $14(35.9)$ & $14(87.5)$ & $\begin{array}{c}357 \\
(73.9) \\
\end{array}$ & $0.0001+$ & 7 \\
\hline $\mathrm{A} 4 \mathrm{~N} 2 \mathrm{M} 1$ & $163(93.7)$ & $81(87.1)$ & $111(95.7)$ & $42(93.3)$ & $35(89.7)$ & $15(93.8)$ & $\begin{array}{c}447 \\
(92.5)\end{array}$ & 0.25 & 8 \\
\hline BINO & $130(74.7)$ & $74(79.6)$ & $61(52.6)$ & $32(71.1)$ & $28(71.8)$ & $14(87.5)$ & $\begin{array}{c}339 \\
(70.2)\end{array}$ & $0.0001+$ & 5 \\
\hline B1N1 & $134(77.0)$ & $70(75.3)$ & $67(57.8)$ & $31(68.9)$ & $26(66.7)$ & $14(87.5)$ & $\begin{array}{c}342 \\
(70.8)\end{array}$ & $0.007+$ & 6 \\
\hline B1N2 & $152(87.4)$ & $75(80.6)$ & $86(74.1)$ & $34(75.6)$ & $29(74.4)$ & $15(93.8)$ & $\begin{array}{c}391 \\
(81.0)\end{array}$ & $0.03+$ & 7 \\
\hline B2NO & $164(94.3)$ & $79(84.9)$ & 103 (8.8.8) & $39(86.7)$ & $39(100)$ & $12(75.0)$ & $\begin{array}{c}436 \\
(90.3)\end{array}$ & $0.004 t$ & 6 \\
\hline $\mathrm{B} 2 \mathrm{~N}_{1}$ & $163(93.7)$ & $81(87.1)$ & $108(93.1)$ & $42(93.3)$ & $38(97.4)$ & $13(81.3)$ & $\begin{array}{c}445 \\
(92.1) \\
\end{array}$ & 0.15 & 7 \\
\hline
\end{tabular}

toms frequently reported. We set out to report the intermediate outcomes of service members with operatively managed low lumbar fractures.

Methods: A retrospective analysis of a surgical database at three military institutions was performed; patients undergoing spine surgery designated in as engaged in Operations Enduring and/or Iraqi Freedom between 01JUL2003 and 01JUL2013 were included. Medical records and radiographs were reviewed for all patients treated with combat-related lumbar burst fractures (L3-L5). We included all patients who underwent operative fixation in theatre or at our institution.

Results: Twenty-four patients with an average age of $28.1 \pm 67.2$ sustained low lumbar (L3-L5) burst fractures. The average injury severity score was 22.6. Six patients sustained gunshot wound(s), fifteen sustained blast injuries from an improvised explosive device, in addition to one crush and one MVA injury. The average number of thoracolumbar levels

ultimate goal of the new the AOSpine Thoracolumbar Spine Injury Classification System was to allow for the development of a globally accepted treatment algorithm for thoracolumbar trauma. The goal of the current study is to establish a surgical algorithm to accompany the AOSpine Thoracolumbar Spine Injury Classification System.

Methods: A survey was sent to AOSpine members from the six AO regions of the world, and surgeons were asked if a patient should undergo an initial trail of conservative management or if surgical management was warranted. The survey consisted of controversial injury patterns. Using the results of the survey, a surgical algorithm was developed.

Results: The AOSpine Trauma Knowledge forum determined that injuries in which less than thirty percent of surgeons would recommend surgical intervention should undergo a trial of nonoperative care, and injuries in which $70 \%$ of surgeons would recommend surgery should undergo surgical intervention (Table 1). Using these thresholds, it was determined that injuries with a Thoracolumbar AOSpine Injury Score (TL AOSIS) of three or less should undergo a trail of conservative treatment, and injuries with a TL AOSIS of more than five should undergo surgical intervention. Operative or non-operative treatment is acceptable for injuries with a TL AOSIS of four or five.

Conclusion: The current algorithm uses a meaningful injury classification and worldwide surgeon input to determine the initial treatment recommendation for thoracolumbar injuries. This allows for a globally accepted surgical algorithm for the treatment of thoracolumbar trauma.

Paper \#44. Outcomes after Operative Management of CombatRelated Low Lumbar Burst Fractures

Peter M. Formby, MD; Scott Wagner, MD; Gregory Van Blarcum, MD; Alfred J. Pisano, MD; Daniel G. Kang, MD; Ronald A. Lehman Jr., MD

\section{Walter Reed National Military Medical Center}

Introduction: Combat-related lumbar burst fractures have been documented at an increased incidence during the Iraq and Afghanistan conflicts. Surgical management of these injury patterns is controversial, with high reoperation rates and persistent symp- injured was $2.9 \pm 1.4$. Nine patients had evidence of neurologic injury, three of which were complete. The average number of levels fused was $4.3 \pm 2.1$ with fixation extending to the pelvis in four patients (17\%). Ten acute postoperative complications occurred; seven required reoperation. One patient required late reoperation for nonunion. Average clinical follow-up was $3.3 \pm 2.2$ years. At latest follow-up, all were retired from military service or were undergoing separation, ten (43\%)experienced persistent bowel/bladder dysfunction, fifteen $(65 \%)$ had persistent neurological symptoms, seventeen (74\%) had documented persistent low back pain, and nineteen $(83 \%)$ had chronic pain.

Discussion: Low lumbar burst fractures are rare injuries with an increased incidence in current combat casualties. Few studies have examined the intermediate outcomes after operative management of these injury patterns. We found a high rate of acute postoperative complications (43\%), low back pain, and a high reoperation rate $(30 \%)$.

Conclusion: At over three years average follow-up, most patients with operatively-treated low lumbar burst fractures had persistent neurological symptoms and chronic pain, suggestive that surgical management of low lumbar burst fractures is fraught with complications. Paper \#45. PROMIS PF CAT outperforms the ODI and SF-36
Physical Function Domain in 1607 Spine Patients

Darrel S. Brodke, MD; Brandon D. Lawrence, MD; William R. Spiker, MD; Ashley M. Neese, BS; Man Hung, PhD

University of Utah Department of Orthopaedics

Introduction: Evidence-based decision-making and identifying value depend on high quality outcomes measures. Several studies have revealed the shortcomings of the ODI and SF36, commonly used in the spine patient population. The NIH funded PROMIS Network has developed domains with excellent psychometric properties. The Physical Function domain, delivered by Computerized Adaptive Testing (PF CAT) is one such option that performs well in the spine patient population, though direct comparisons with legacy measures have not been performed. This study directly compares 


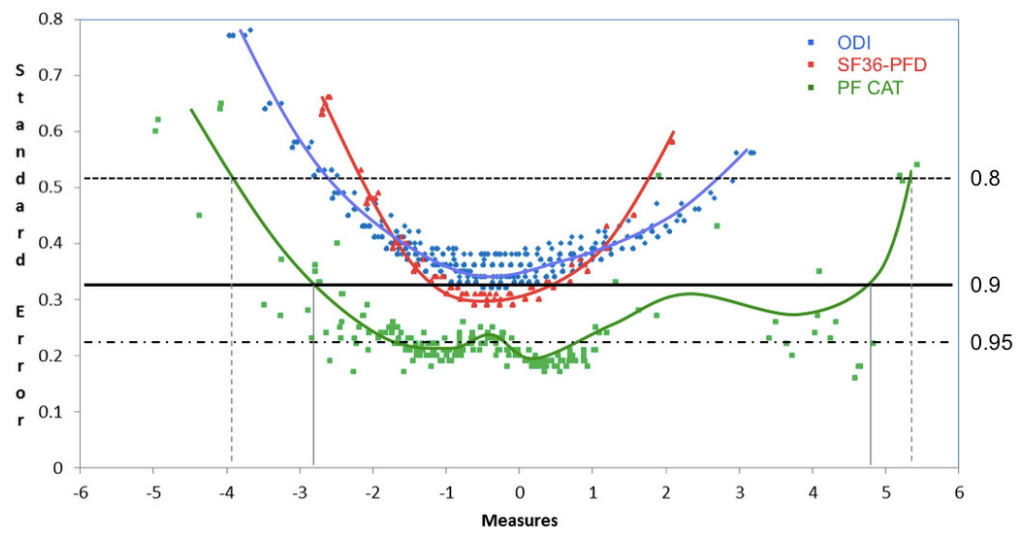

the psychometric performance of the PROMIS PF CAT to the ODI and the SF36 Physical Function Domain (SF-36 PFD) in the same patient population, and aims to generate conversion equations between scores for cross utilization.

Methods: Standard Rasch analysis was performed to directly compare the psychometrics of the PF CAT, ODI, and SF36 PFD. Regression analyses were then performed to predict the PF CAT scores from the ODI and SF36 PFD and vice versa. Pearson correlations were computed to compare the actual and predicted scores for each.

Results: 1,607 patients completed all assessments. The number of questions answered (4.15 vs 9.29/9.96) and time to answer them (44" vs 169"/99") was significantly shorter for the PF CAT, as compared with the ODI and the SF-36PFD. The ceiling and floor effects were excellent for the PF CAT $(0.81 \%$ and $3.86 \%)$, while the ceiling effects were marginal $(6.91 \%$ and $5.97 \%)$ and the floor effects were quite poor (44.24\% and $23.65 \%$ ) for the ODI and SF-36 PFD, respectively. All legacy scale scores significantly predicted the PROMIS PF CAT scores ( $\mathrm{p}<0.0001)$, with excellent correlation for the PF CAT and ODI (0.731) and PF CAT and SF-36 PFD (0.777), allowing use of conversion equations to predict scores, which were generated.

Discussion and Conclusion: The PROMIS PF CAT outperforms the ODI and SF-36 PFD in the spine patient population. It has better coverage, while taking less time to administer with fewer questions to answer. The PF CAT can be accurately predicted from either the ODI or SF-36 PFD scores, allowing conversion from one to the other when comparing older collected data to new scores.

Paper \#46. A Comparison of Surgical Outcomes between Minimally Invasive and Open Thoracolumbar Corpectomy: A Minimum of Two-Year Follow-up

Ehsan Tabaraee, MD; Junyoung Ahn, BS; Khaled Aboushaala, $M D$; Kern Singh, $M D$

\section{Rush University Medical Center}

Introduction: Few studies have assessed the differences in surgical outcomes between minimally invasive (MIS) and open thoracolumbar corpectomies for oncologic, infectious, and traumatic conditions.

Methods: Retrospective review of 33 patients with at least twoyear follow-up who underwent either MIS or open thoracolumbar corpectomy by a single surgeon between 2005-2012 was performed. Patients were stratified based upon approach: Anterior-Posterior MIS (AP MIS), Anterior-Posterior Open (AP), and Posterior Open (P). Narcotic consumption was converted to oral morphine equivalents (OME). Demographics, comorbidity (CCI), location of pathology, peri- and post-operative outcomes, duration of follow-up, and radiographic analyses were assessed. Pearson's Chi-squared test was performed for categorical variables and Student's T-test was performed for continuous data. P-value of $<0.05$ denoted statistical significance.

Results: 33 patients underwent AP MIS, AP, or $\mathrm{P}$ thoracolumbar corpectomy (AP MIS 39.4\%; AP 15.2\%; $\mathrm{P} 45.5 \%)$. AP MIS patients were younger than $\mathrm{P}$ patients $(45.1 \pm 16.2$ vs. $59.3 \pm 17.3$ years; $p<0.05)$ with a lower comorbidity burden than the open cohorts (AP MIS $4.15 \pm 2.7$ vs. AP $9.20 \pm 6.6$ and $\mathrm{P} 7.8 \pm 4 ; \mathrm{p}<0.05$ ). The AP MIS cohort incurred decreased procedural time, blood loss, and hospitalization. AP MIS patients demonstrated lower transfusion rates than the $\mathrm{P}$ cohort $(15.4 \%$ vs. $80 \% ; \mathrm{p}<0.05)$. Post-operative narcotic consumption $(1095.5 \pm 745.3$ vs. $540.3 \pm 259.3 ; \mathrm{p}<0.05)$ and re-operation rate $(40 \%$ vs. $0 \% ; \mathrm{p}<0.05)$ were significantly greater in the AP cohort. Although the difference was not statistically significant, infection rates were $0 \%, 13.3 \%$, and $20 \%$ in the AP MIS, P and AP cohorts, respectively. No differences in BMI, intra-operative IV fluids, post-operative transfusions, complications, or ICU admissions were observed between cohorts. All patients demonstrated fusion at 1-year based upon computed tomography.

Discussion: The AP MIS patients demonstrated decreased procedural time, hospitalization, blood loss, and narcotic consumption. In addition, the AP MIS patients demonstrated a lower re-operation rate as compared to AP open patients. All cohorts demonstrated similar fusion rates while the open cohorts demonstrated greater infection rates.

Conclusions: MIS thoracolumbar corpectomy appears to decrease post-operative complications, narcotic consumption, and hospitalization while maintaining successful arthrodesis rates with at least two-year follow-up.

\begin{tabular}{|c|c|c|c|c|c|}
\hline & $\begin{array}{l}\text { Posterior } \\
\text { Open }\end{array}$ & p-value & AP MIS & p-value & AP Open \\
\hline Count (n) & 15 & & 13 & & 5 \\
\hline$\%$ Female & $53.3 \%$ & 0.4 & $69.2 \%$ & 0.7 & $60 \%$ \\
\hline Age (years) & $59.3 \pm 17.3$ & $<0.05$ & $45.1 \pm 16.2$ & 0.1 & $59.6 \pm 8.6$ \\
\hline BMI & $24.4 \pm 2.5$ & 0.4 & $26.9 \pm 5.8$ & 0.5 & $24.6 \pm 2.1$ \\
\hline CCI & $7.8 \pm 4$ & $<0.05$ & $4.15 \pm 2.7$ & $<0.05$ & $9.20 \pm 6.6$ \\
\hline \multicolumn{6}{|l|}{ Primary Diagnosis: } \\
\hline Tumor & $73.3 \%(\mathrm{n}=11)$ & & $46.2 \%(\mathrm{n}=6)$ & & $60 \%(n=3)$ \\
\hline Trauma & $6.7 \%(\mathrm{n}=1)$ & & $46.2 \%(\mathrm{n}=6)$ & & $40 \%(\mathrm{n}=2)$ \\
\hline Infection & $20.0 \%(\mathrm{n}=3)$ & & $7.6 \%(\mathrm{n}=1)$ & & $0 \%$ \\
\hline Metastatic Tumor & $54.5 \%{ }^{4}$ & 0.3 & $66.7 \%^{5}$ & 0.4 & $60 \%^{4}$ \\
\hline \multicolumn{6}{|l|}{ Last Clinical Follow-up Period (Years) } \\
\hline Mean & $1.8 \pm 2.4^{8}$ & & $1.5 \pm 1.0^{8}$ & & $4.8 \pm 3.2^{8}$ \\
\hline Range & $(0.1-6.9)$ & & $(0.2-3.0)$ & & $(1.0-7.8)$ \\
\hline Fusion Rate (CT Scan 1 Year) & $100 \%$ & - & $100 \%$ & - & $100 \%$ \\
\hline \multicolumn{6}{|c|}{ Peri-Operative Outcomes } \\
\hline Procedural time (Hours) & $6.0 \pm 1.8$ & $<0.05$ & $3.9 \pm 1.1$ & $<0.05$ & $6.1 \pm 2.5$ \\
\hline EBL $(\mathbf{c c})$ & $2275 \pm 1529$ & $<0.05$ & $400 \pm 345.1$ & $<0.05$ & $2260 \pm 2039.1$ \\
\hline Mean LOS (Days) & $11.4 \pm 7.8$ & $<0.05$ & $5.5 \pm 2.2$ & $<0.05$ & $9 \pm 3.7$ \\
\hline $\begin{array}{c}\text { Mean Total OME } \\
\text { (Oral Morphine Equivalent) }\end{array}$ & $\begin{array}{l}1214.3 \pm 1408 \\
6\end{array}$ & 0.1 & $540.3 \pm 259.3$ & $<0.05$ & $1095.5 \pm 745.3$ \\
\hline Intra-Operative IV Fluids & $\begin{array}{c}3921.4 \pm 1228 . \\
0\end{array}$ & 0.9 & $\begin{array}{l}3753.8 \pm 1007 \\
2\end{array}$ & 0.7 & $4000 \pm 2222.6$ \\
\hline Transfusion Rate & $80 \%$ & $<0.05$ & $15.4 \%$ & 0.1 & $80 \%$ \\
\hline \multicolumn{6}{|l|}{$\begin{array}{l}\text { Amount Transfused Intra-Operatively } \\
\text { (Units): }\end{array}$} \\
\hline PRBCs & $2.6 \pm 2.8$ & $<0.05$ & $0.5 \pm 1.1$ & 0.1 & $1.8 \pm 2.5$ \\
\hline FFP & $0.6 \pm 0.8$ & $<0.05$ & 0 & 0.1 & $0.4 \pm 0.8$ \\
\hline Platelets & $0.3 \pm 0.7$ & 0.1 & 0 & - & 0 \\
\hline \multicolumn{6}{|l|}{ Amount Transfused Post-Operatively } \\
\hline $\begin{array}{l}\text { (Units): } \\
\text { PRBCs }\end{array}$ & $1.1 \pm 2.3$ & 0.4 & $0.3 \pm 0.8$ & 0.4 & 0 \\
\hline Intra-Operative Complication Rate & $0 \%$ & $\cdot$ & $0 \%$ & 0.1 & $20 \%^{3}$ \\
\hline In-Hospital Complication Rate & $14.3 \%^{1}$ & 0.6 & $7.7 \%^{2}$ & 0.5 & $0 \%$ \\
\hline Post-Operative Infection Rate & $13.3 \%$ & 0.2 & $0 \%$ & 0.1 & $20 \%$ \\
\hline Reoperation Rate & $13.3 \%^{6}$ & 0.2 & $0 \%$ & $<0.05$ & $40 \%{ }^{7}$ \\
\hline
\end{tabular}


Paper \#47. Can Triggered Electromyography Monitoring Throughout Retraction Predict Postoperative Symptomatic Neuropraxia after XLIF? Results from a prospective multicenter trial.

Jim A. Youssef, MD; Juan S. Uribe, MD; Robert E. Isaacs, MD

Spine Colorado

Background: This multicenter study aims to evaluate the utility of triggered electromyography (t-EMG) recorded throughout psoas retraction during lateral transpsoas interbody fusion to predict postoperative changes in motor function.

Methods: Three hundred twenty three (323) patients undergoing L4-5 minimally invasive lateral interbody fusion from 21 sites were enrolled. Intraoperative data collection included initial t-EMG thresholds of the posterior blade from the stimulated retractor and subsequent t-EMG threshold values collected every five minutes throughout retraction. Additional data collection included dimensions/duration of retraction as well as pre-and postoperative lowerextremity neurologic exams.

Results: Prior to expanding the retractor, the lowest t-EMG threshold was identified posterior to the retractor in $94 \%$ of cases. Postoperatively, $13(4.5 \%)$ patients had a new motor weakness that was consistent with symptomatic neuropraxia (SN) of lumbar plexus nerves on the approach side. There were no significant differences between patients with or without a corresponding postoperative $\mathrm{SN}$ with respect to initial posterior blade reading $(\mathrm{p}=0.600)$, or retraction dimensions $(p>0.05)$. Retraction time was significantly longer in those patients with SN vs. those without $(p=0.031)$. Stepwise logistic regression showed a significant positive relationship between presence of new postoperative $\mathrm{SN}$ and total retraction time $(\mathrm{p}<0.001)$, as well as change in t-EMG thresholds over time $(\mathrm{p}<0.001)$.

Conclusions: Prolonged retraction time and increasing t-EMG thresholds throughout retraction are predictors of declining nerve integrity. In addition to a careful approach with minimal muscle retraction and consistent lumbar plexus directional retraction using dynamically triggered discrete-threshold t-EMG, the incidence of postoperative motor neuropraxia may be reduced by limiting retraction time and utilizing t-EMG consistently throughout retraction.

Paper \#48. Timing of Postoperative Complications After Lumbar Fusion Procedures

Daniel D. Bohl, MPH; Andre M. Samuel, BBA; Matthew L. Webb, BA; Adam M. Lukasiewicz, MSc; Bryce A. Basques, BS; Nicholas $S$. Golinvaux, BA; Junyoung Ahn, BS; Kern Singh, MD; Alexander R. Vaccaro, MD, PhD; Jonathan N. Grauer, MD

\section{Yale School of Medicine}

Introduction: There is little published description of the timing of post-operative complications following spinal surgery. As such, the purpose of this study is to characterize the timing of postoperative complications after lumbar fusion procedures utilizing a sample of patients undergoing these procedures at community and academic centers nationwide.

Methods: Patients undergoing lumbar fusion procedures during 2010-2012 were identified in the American College of Surgeons National Surgical Quality Improvement Program (NSQIP) database. The NSQIP is a database that prospectively follows patients for the development of postoperative complications during the 30 days after surgery. Analysis of this data included identification of the median day (and interquartile range) after surgery for diagnosis of each complication. The proportion of each complication diagnosed before versus after discharge was also determined.
Results: A total of 7,908 patients undergoing lumbar fusion procedures were identified in the NSQIP database.

Figure 1 demonstrates that the median postoperative day (and interquartile range) for diagnosis of anemia requiring transfusion was $0(0-1)$, myocardial infarction 2 (1-5), renal insufficiency 3 (2-7), pneumonia 4 (3-6), stroke 4 (2-9), pulmonary embolism 5 (4-11), urinary tract infection 6 (3-13), deep vein thrombosis 7 (5-13), sepsis 8 (3-16), and surgical site infection 16 (12-21).

The proportion of complications occurring after (versus before) discharge for anemia requiring transfusion was $1 \%$, myocardial infarction $24 \%$, renal insufficiency $24 \%$, pneumonia $22 \%$, stroke $36 \%$, pulmonary embolism $31 \%$, urinary tract infection $52 \%$, deep vein thrombosis $59 \%$, sepsis $60 \%$, and surgical site infection $91 \%$.

Discussion: These precisely described post-operative time periods among this recent, large, national sample enable heightened clinical awareness amongst clinicians. This study also demonstrates that databases that only collect data prior to patient discharge fail to capture a large proportion of the later occurring complications, including urinary tract infection, deep vein thrombosis, sepsis, and surgical site infection.

Conclusion: Clinicians should have a low threshold for work-up of these complications during the specific post operative time periods identified here. 


\section{RF Paper \# 1. Primary and Revision Posterior Lumbar Fusion Have Similar General Health Complication Rates}

Bryce A. Basques, BS; Adam M. Lukasiewicz, MSc; Matthew L. Webb, AB; Andre M. Samuel, BBA; Nicholas Golinvaux, BA; Daniel D. Bohl, MPH; Jonathan Newman Grauer, MD

\section{Yale School of Medicine}

Introduction: Revision lumbar fusions are relatively common following primary lumbar fusions. Previous studies have described an increased risk of postoperative complications following revision lumbar fusion relative to primary cases, however these studies have generally used small, single-institution cohorts. There is a need to validate these findings using a large, national cohort of patients in order to have an accurate assessment of the relative risk of revision posterior lumbar fusion compared to primary surgery.

Methods: The prospectively-collected American College of Surgeons National Surgical Quality Improvement Program (ACSNSQIP) database was used to identify patients that underwent

Table 1. Comparison of complication rates between primary and revision posterior lumbar fusion.

\begin{tabular}{|c|c|c|c|c|c|c|}
\hline & \multirow{2}{*}{$\begin{array}{c}\text { Primary } \\
\text { procedure }\end{array}$} & \multirow{2}{*}{$\begin{array}{l}\text { Revision } \\
\text { procedure }\end{array}$} & \multicolumn{2}{|c|}{ Bivariate analysis } & \multicolumn{2}{|c|}{ Multivariate analysis* } \\
\hline & & & $\mathrm{RR}$ & $p$ & $\mathrm{RR}$ & $p$ \\
\hline Any complication & $9.0 \%$ & $8.1 \%$ & 0.9 & 0.430 & 0.8 & 0.217 \\
\hline Severe complication & $7.0 \%$ & $6.0 \%$ & 0.9 & 0.320 & 0.8 & 0.168 \\
\hline Death & $0.3 \%$ & $0.1 \%$ & 0.4 & 0.468 & 0.5 & 0.444 \\
\hline Coma $>24$ hours & $0.1 \%$ & $0.0 \%$ & - & - & - & - \\
\hline Ventilator $>48$ hours & $0.4 \%$ & $0.0 \%$ & - & - & - & . \\
\hline Unplanned intubation & $0.5 \%$ & $0.0 \%$ & - & - & - & . \\
\hline Stroke/cerebrovascular accident & $0.2 \%$ & $0.3 \%$ & 1.6 & 0.566 & 1.5 & 0.587 \\
\hline Thromboemolic event (DVT/PE) & $1.1 \%$ & $0.9 \%$ & 0.8 & 0.613 & 0.8 & 0.548 \\
\hline Surgical site infection & $2.3 \%$ & $1.6 \%$ & 0.7 & 0.200 & 0.6 & 0.133 \\
\hline Sepsis/septic shock & $1.1 \%$ & $0.8 \%$ & 0.7 & 0.385 & 0.6 & 0.248 \\
\hline Cardiac arrest requiring CPR & $0.2 \%$ & $0.0 \%$ & - & - & - & - \\
\hline Myocardial Infarction & $0.3 \%$ & $0.1 \%$ & 0.4 & 0.406 & 0.4 & 0.396 \\
\hline Acute renal failure & $0.1 \%$ & $0.0 \%$ & - & - & - & . \\
\hline Return to the operating room & $3.5 \%$ & $3.3 \%$ & 0.9 & 0.706 & 0.9 & 0.542 \\
\hline Wound dehiscence & $0.4 \%$ & $0.1 \%$ & 0.4 & 0.332 & 0.3 & 0.263 \\
\hline Minor complication & $2.9 \%$ & $2.4 \%$ & 0.8 & 0.397 & 0.8 & 0.272 \\
\hline Urinary tract infection & $1.4 \%$ & $1.0 \%$ & 0.9 & 0.809 & 0.9 & 0.624 \\
\hline Pneumonia & $0.8 \%$ & $0.4 \%$ & 0.5 & 0.232 & 0.5 & 0.199 \\
\hline Progressive renal insufficiency & $0.1 \%$ & $0.0 \%$ & - & - & - & \\
\hline Peripheral nerve injury & $0.1 \%$ & $0.1 \%$ & 0.9 & 0.961 & 1.0 & 0.989 \\
\hline Blood transfusion & $12.3 \%$ & $22.3 \%$ & 2.1 & $<0.001$ & 2.0 & $<0.001$ \\
\hline Readmission & $5.4 \%$ & $5.2 \%$ & 1.0 & 0.897 & 1.0 & 0.846 \\
\hline
\end{tabular}

Bolding indicates statistical significance $(\mathrm{p}<0.05) . \mathrm{RR}=$ Relative risk.

*Each line represents a separate multivariate analysis for each variable in order to give an adjusted $\mathrm{OR}$ and $\mathrm{p}$-value by controlling for all demographics, comorbidities, and operative approaches found in Table 1 and Table 2.

undergoing primary and revision posterior lumbar fusion from 2005 to 2013. The occurrence of individual and aggregated postoperative complications within 30 days was compared between primary and revision procedures using multivariate Poisson regression with robust error variance to control for patient demographics and operative characteristics such as operative time and number of levels. The rates of blood transfusion and readmission were also compared between primary and revision procedures.

Results: Of the 7,284 posterior lumbar fusion procedures that met inclusion criteria, 763 (10.4\%) were revision cases. There were no differences in the rates of 30-day postoperative complications between primary and revision posterior lumbar fusion using multivariate analysis that controlled for patient and operative characteristics (Table 1). There was also no difference in readmission rates. The only significant difference found was an increased rate of blood transfusion in revision surgery (RR 2.0, p < 0.001).

Conclusions: Using 7,284 patients from a prospectively-collected national surgical registry, this study suggests that revision posterior lumbar fusion does not carry significantly increased risk of general health com- plications or readmission compared to a primary posterior lumbar fusion. Patients undergoing revision surgery were twice as likely to receive a blood transfusion. This information suggests that general health risk stratification for revision procedures can be similar to that considered for primary cases.

\section{RF Paper \# 2. Incidental Durotomy Increases The Risk For Surgical Site Infection In Lumbar Spine Surgery In Both Obese And Non-obese Patients}

Christopher A. Burks, MD, MS; Brian C. Werner, MD; Scott Yang, MD; Adam L. Shimer, M.D.

\section{University of Virginia}

Objectives: Incidental durotomy is a relatively common surgical complication in lumbar spine surgery. Obesity is associated with an increased risk of incidental durotomy and surgical site infection; however the combined impact of incidental durotomy and obesity on surgical site infection following lumbar spine surgery is unknown. The objective of this study was to utilize a large population database to determine the impact of obesity and incidental durotomy on surgical site infection in lumbar spine surgery.

Methods: Patients who underwent lumbar spine surgery were identified in the PearlDiver Patient Records Database (www. pearldiverinc.com) using the Current Procedural Terminology (CPT) codes for lumbar decompression, instrumentation, and revision decompression procedures. They were then stratified into separate cohorts based on ICD-9 codes for obesity, as measured by body mass index (BMI), procedural groups for decompression, instrumentation with or without decompression, revision decompression, and documented incidental durotomy by ICD-9 codes for durotomy. These groups were then separately queried for the documentation of surgical site infection by ICD-9 code and CPT codes for incision and debridement of the lumbar spine in the six months following the index procedure. Patient demographics and comorbidities were assessed. Odds ratios and 95\% confidence intervals were calculated and chi square test was used to assess for statistical significance [Table 1].

Results: The incidence of durotomy was $0.7-2.3 \%$ out of a total of 303,476 lumbar spine procedures. The incidence of surgical site infection was $1.2-11.1 \%$ in the no durotomy group and $3.4-15.0 \%$ in the durotomy group. The lowest rate of infection was in the non-obese, decompression, without durotomy group $(1.2 \%)$ and the highest rate was in the obese, revision, durotomy group $(15.0 \%)$. There was a statistically significant increase in the incidence of surgical site infection when associated with incidental durotomy in all groups except for revision surgery in the obese patient $(\mathrm{p}=0.186, \mathrm{OR}=1.4,95 \% \mathrm{CI}[0.9-2.3])$. The rate of durotomy and surgical site infection was higher for all procedural groups in the obese patients.

Conclusions: This analysis of a large administrative database demonstrates that incidental durotomy and obesity are associated

\begin{tabular}{|c|c|c|c|c|c|c|c|c|c|c|}
\hline \multicolumn{9}{|c|}{ Non-Obese $($ BMI < 30) } & \multicolumn{2}{|c|}{ Statistical Analysis } \\
\hline & Total $\#$ & \#No Drootomy & $=$ Durotomy & \% Durotomy & \multicolumn{2}{|c|}{$\begin{array}{c}=(\%) \text { Infection No } \\
\text { Durotomy }\end{array}$} & \multicolumn{2}{|c|}{$\begin{aligned}= & (\%) \text { Infection } \\
& \text { Durotomy }\end{aligned}$} & Odds Ratio [95\%CI.] & $P$ \\
\hline Decompression & 166,341 & 165,173 & 1,168 & $0.7 \%$ & 1,970 & (1.2\%) & 40 & $(3.4 \%)$ & $2.9[2.1-4.0]$ & $<0.0001$ \\
\hline Fusion & 56,887 & 56,144 & 743 & $1.3 \%$ & 1,910 & (3.4\%) & 42 & $(5.7 \%)$ & $1.7[12-2.3]$ & 0.001 \\
\hline Revision Procedure & 25,903 & 25,479 & 424 & $1.6 \%$ & 1,021 & $(4.0 \%)$ & 28 & $(6.6 \%)$ & $1.7[1.1-2.5]$ & 0.010 \\
\hline \multicolumn{9}{|c|}{ Obese $($ BMI > 30) } & \multicolumn{2}{|c|}{ Statistical Analysis } \\
\hline & Total $\#$ & $=$ No Drwotomy & \#Durotomy & $\%$ Durotomy & \multicolumn{2}{|c|}{$\begin{array}{c}=(\%) \text { Infection } N o \\
\text { Durotomy }\end{array}$} & \multicolumn{2}{|c|}{$\begin{aligned} \text { \# } & (\%) \text { Infection } \\
& \text { Durotomy }\end{aligned}$} & OddsRatio [95\%CI] & $P$ \\
\hline Decompression & 35,225 & 34,865 & 360 & $1.0 \%$ & 1,514 & $(4.3 \%)$ & 25 & $(6.9 \%)$ & $1.6[1.1-2.5]$ & 0.023 \\
\hline Fusion & 12,921 & 12,710 & 211 & $1.6 \%$ & 1,073 & $(8.4 \%)$ & 30 & $(14.2 \%)$ & $1.8[12-2.7]$ & 0.004 \\
\hline Revision Procediere & 6,199 & 6,059 & 140 & $2.3 \%$ & 671 & (11.1\%) & 21 & $(15.0 \%)$ & $1.4[0.9-2.3]$ & 0.186 \\
\hline
\end{tabular}


with an increased rate of surgical site infection in lumbar spine surgery. Incidental durotomy though relatively common may not be as benign a long-term complication as once thought.

\section{RF Paper \# 3. The Effect of Sustained Localized Delivery of E. Coli-expressed rhBMP-2 on Bone Formation in a Lumbar Spine Facet Fusion Model}

Kevin Baker, Ph.D.; Abigail Davidson, BS; Tristan Maerz, M.S. Eng; Daniel Park, MD; Jeffrey S. Fischgrund, MD

\section{Beaumont Health System}

Introduction: Physical adsorption of rhBMP-2 on a collagen sponge results in poor protein localization, ultimately leading to burst release of rhBMP-2 which may result in adverse effects. Incorporation of rhBMP-2 into a biomimetic calcium phosphate $(\mathrm{CaP})$ coating results in extended release of the growth factor in a localized fashion. The goal of this study is to compare in vivo bone formation associated with low doses ( $2.5 \mathrm{ug}$ per side) of E. Coli-expressed rhBMP-2 when physically-adsorbed on a collagen sponge, or incorporated in a CaP coating.

Methods: Twenty New Zealand White Rabbits underwent a posterior approach to the lumbar spine. Posterior elements were reached and the bifurcated central spinous processes, including facet joints were decorticated before implanting collagen sponges bilaterally bridging the L5-L6 level. For animals in Group I, E. Coli-expressed rhBMP-2 was added to the collagen sponge immediately prior to the implantation (Group A1= no BMP-2, A2=2.5 ug BMP-2), while in Group II the BMP-2 was incorporated in a calcium phosphate coating deposited on the collagen sponge $(\mathrm{B} 1=$ no $\mathrm{BMP}-2, \mathrm{~B} 2=2.5$ ug BMP-2). Rabbits were sacrificed at 8 weeks and the spines were excised for manual, histological and CT evaluation.

Results: All animals tolerated the procedure well, with rapid recovery from anesthetic. The qualitative analysis of in vivo bone formation is listed in Table 1. Briefly, control specimens (Group A1) demonstrated minimal facet hyperplasia, with minimal bone formation. Animals that received sponges with $2.5 \mathrm{ug}$ of adsorbed BMP-2 demonstrated significant facet hyperplasia and new bone formation. Sponges with a CaP coating without BMP-2 showed significant facet hyperplasia and solid fusion in some specimens. When $2.5 \mathrm{ug}$ of BMP-2 was incorporated into the $\mathrm{CaP}$ coatings, significant facet hyperplasia and new bone formation was observed.

Discussion: The facet fusion technique represents a reproducible model with minimal soft tissue trauma, when compared to a traditional inter-transverse process fusion. While the incorporation of low dose BMP-2 into a CaP coating did not provide clearly superior results to physically adsorbed $\mathrm{BMP}-2$, the presence of a $\mathrm{CaP}$ coating without BMP-2 led fusion in several animals. More studies are underway to investigate and compare higher doses of BMP-2.

\section{RF Paper \# 4. An Analysis Of Early-crossovers In A Cohort Of Patients Receiving Non-operative Care}

Anurekha Ramakrishnan, MS in Statistics; Matthew C. Cowperthwaite, PhD; Michael Webb, MD

NeuroTexas Institute Research Foundation, St. David's HealthCare

Introduction: For patients with lumbar disc herniation, the decision of whether and when to opt for surgical treatment is not always obvious; clinicians often face similar uncertainty in their recommendations. Here, we study crossover patients in a cohort randomized to a prolonged non-operative care protocol to study factors affecting the treatment course and outcomes following surgery.

Methods: Individual EQ-5D patient utility measures were obtained at baseline, 2, 4, 8, 12, 26, 38, and 52 weeks for 137 patients randomized to a prolonged conservative care treatment protocol. Crossover patients $(n=44 ; 32.1 \%)$ were defined as those electing to undergo surgery during the one-year observation period. Utility scores at each observation point were summarized using kernel-density estimators. Crossover and non-crossover groups were compared using Kruskal-Wallis tests; Chi-square tests were used to compare outcomes between groups. Decision trees and logistic regression were used to predict patient outcomes.

Results: In our cohort, 40/44 (90.9\%) of crossover events occurred in the first six months of treatment; 39/44 (88.6\%) patients reported some health problems $(\mathrm{EQ}-5 \mathrm{D} \leq .86)$ just before surgery. Early crossovers (before 3 months) had lower mean utility at baseline than non-crossovers, greater recovery by 26 and 52 weeks, and were associated with better one-year outcomes than non-crossovers with similar baseline utility $(\mathrm{p}<0.05)$. Decision trees revealed that predictors of a crossover event were lower baseline utility and slower improvement during the first 8 weeks of treatment. Logistic regression and decision tree models were able to predict one-year patient outcomes as early as 8 weeks with $76 \%$ accuracy.

Discussion:This study suggests that, for patients not initially responding to non-operative treatment, earlier surgery is associated with faster recovery and better one-year outcomes. Our results suggest that surgical decisions are predictable, although there were insufficient crossovers to predict outcomes after surgery.

Conclusion: Early surgery may be beneficial for patients not responding to non-operative care; such patients can be identified as early as 8 weeks. However, given the small sample size, our results motivate a broader investigation into optimal timing and outcomes of surgical treatment for lumbar discectomy.

\section{RF Paper \# 5. Osteoporosis Increases Radiographic Failure After Transforaminal Lumbar Interbody Fusion}

Peter M. Formby, MD; Scott Wagner, MD; Daniel G. Kang, MD; Melvin Helgeson, $M D$

Walter Reed National Military Medical Center

Background: Osteoporosis may have negative effects on outcomes after transforaminal lumbar interbody fusion (TLIF), and vertebral body bone mineral density (BMD) may be an important determinant of complication rates. However, to our knowledge, there has never been a definitive assessment comparing radiographic outcomes in TLIF patients with and without osteoporosis.

Methods: A retrospective review at a single institution over a ten year period. We included all patients over age 50 following TLIF with imme- 
diate post-operative and follow-up computed tomography (CT) scans available for radiographic quantification of interbody cage subsidence. Using computed tomography (CT) Hounsfield Unit (HU) measurements, patients were stratified as either osteoporotic or non-osteoporotic, with significant HU cutoff values determined previously at our institution. Plain radiographs and CT scans were evaluated for evidence of implant subsidence, migration, fusion, vertebral body fracture or loosening of posterior fixation.

Results: We identified 128 patients with complete CT imaging and average 26.8 month follow up. Males comprised $60.5 \%$ and females $39.5 \%$ of patients, with an average age of 59 years, and $19.4 \%$ of all patients classified as osteoporotic. However, the average age for patients with osteoporosis was 66.2 years, versus 59.7 years for patients without osteoporosis $(\mathrm{p}=0.0004)$. The rate of implant subsidence was $56.0 \%$ in osteoporotic patients (average subsidence $5.8 \mathrm{~mm}$ ), compared to $43.8 \%$ in patients with non-osteoporotic HU values (average subsidence $5.5 \mathrm{~mm}$ ). Implant migration occurred in $8.3 \%$ versus $4.2 \%$ of osteoporotic and non-osteoporotic patients, respectively. Evidence of loosening of posterior fixation was noted in $16.7 \%$ of patients with osteoporosis, compared to $7.4 \%$ in non-osteoporotic patients. The fusion rate was $57 \%$ in osteoporotic versus $56 \%$ in non-osteoporotic patients. Lastly, vertebral fractures were diagnosed in $8.3 \%$ of osteoporotic patients, as opposed to $1.1 \%$ of non-osteoporotic patients.

Discussion: Lumbar osteoporosis determined through CT HU demonstrated an increased rate of radiographic failure after TLIF, with a nearly doubled rate of pedicle screw loosening, vertebral fracture and implant migration.

Conclusion: Our study highlights the utility of CT HU to identify osteoporotic patients at higher risk for implant failure following TLIF, and should prompt careful attention to maintain endplate competence and optimize surgical technique during instrumentation.

\section{RF Paper \# 6. Are Preoperative MRI Findings Predictors For Revision Surgery? An Analysis Of Revision Surgery And Patient Satisfaction Following Single Level Posterior Lumbar Fusion With Minimum 5 Year Follow-up}

William D. Long III, MD; Lukas Lampe, MD; Janina Kueper, BA; Benjamin Bjerke-Kroll, MD; Joseph T. Nguyen, MPH; Federico P. Girardi, MD; Andrew A. Sama, MD; Frank P. Cammisa Jr, MD; Alexander P. Hughes, MD
$(74.5 \%)$ stated they had moderate or complete relief of their preoperative symptoms, and 4 patients $(7.8 \%)$ reported no benefit from surgery. There were no differences in back pain $(\mathrm{P}=0.479)$, leg pain $(\mathrm{P}=0.154)$, and ODI scores $(\mathrm{P}=0.953)$. Preoperative MRI at the operative and adjacent levels were not independent risk factors for subsequent revision surgery. Patients with revision surgery on their supra-adjacent segment had higher mean values on disc endplate Modic score, disk degeneration Pfirrmann grade, total endplate score, and size of herniation on preoperative MRI (NS). A stepwise, multivariable regression model identified younger age $(\mathrm{P}=0.004)$ and decreased sacral slope as increased risk factors for revision $(\mathrm{P}=0.019)$. The greatest risk factor for revision surgery was the presence of deformity $(\mathrm{OR}=13.764)$.

Conclusions: Single-level PSF for degenerative conditions of the lumbar spine provides favorable outcomes in the majority of patients at five years. Preoperative MRI degenerative findings may not be predictive for identifying patients likely to undergo revision surgery for supra-adjacent segment degeneration. Younger age, decreased sacral slope and the presence of spinal deformity may increase the risk for subsequent surgery within five years of PSF or PLIF. Further studies of larger cohorts are warranted to investigate differences in imaging findings as potential risk factors.

RF Paper \# 7. BMP-2 Dose Response Curves for Interbody Fusions: Low Doses of BMP-2 achieve High Fusion Rates

Arjun S. Sebastian, MD; Jad Khalil, MD; Mark Dekutoski, MD; Ahmad Nassr, MD

\section{Mayo Clinic}

Introduction: Bone Morphogenetic Protein-2 (BMP-2) is an FDA approved device for interbody fusion when used with a specific brand of Anterior Lumbar Interbody Fusion (ALIF) cage. Off-label use of BMP-2 has seen widespread application across the spectrum of spine fusion procedures. It is believed that delivery of clinically effective Minimal Access Surgery (MAS) interbody fusion procedures is dependent upon the use of biologics. While assumed and widely practiced, the BMP-2 dose-response curve for effective fusion occurrence has not been defined. The use of BMP-2 has received scrutiny due to direct and potential side effects as well as cost.

The purpose of this study is to determine the minimum BMP
Hospital for Special Surgery

Introduction: There is limited data on the longterm effectiveness of single-level posterior spinal fusion (PSF) or posterior lumbar interbody fusion (PLIF) in providing durable symptom and disability relief for the degenerative lumbar spine. Outcomes in patients who undergo lumbar fusion surgery are highly variable. This study examined the reoperation rate after single-level PSF or PLIF in patients with minimum 5 year follow-up. Differences in demographics, magnetic resonance imaging (MRI), radiographic findings, and outcomes between patients who did and did not undergo subsequent spine surgery were analyzed.

Methods: The medical records, radiographs, and MRI of patients who underwent single-level PSF or PLIF at a single institution between January 2007 and August 2008 were reviewed. Differences in demographics, preoperative MRI findings and outcome data were analyzed. Longterm follow-up of patient satisfaction and function was assessed via a telephone questionnaire that queried pain, work status, and the Oswestry Disability Index (ODI). Fifty one patients participated.

Results: Six $(11.8 \%)$ of patients underwent revision within five years of index surgery. Thirty eight patients

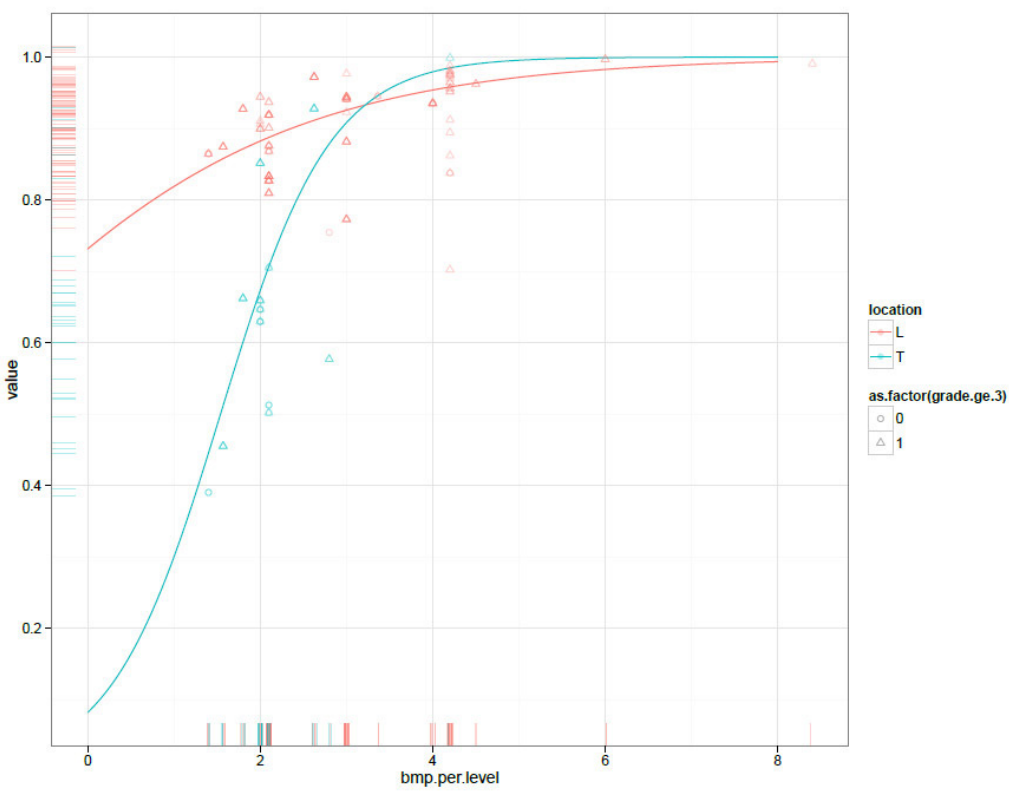


dose needed to achieve satisfactory fusion. Knowledge of this dose could reduce consumption and thus decrease cost and potential side effects related to its use.

Methods: Thirty-five patients who underwent Direct Lateral Interbody Fusions (DLIF) or a combination of DLIF and ALIF at 144 levels were retrospectively reviewed. Fusion across levels was graded by 3 independent surgeons using a novel Computed Tomography (CT) based grading system (fusion grades 0-3). The BMP dose used at each level was recorded from OR and supply chain records. Correlation between BMP dose and fusion grade was measured.

Results: The average patient radiographic follow-up was 12.24 months. The overall fusion (grade 2,3) rate (full bridging consolidation) was $91.7 \%$ (132/144 levels). Non-union, suspected non-union or incomplete union (grade 0-1) rate was 8.3\% (12/144 levels). Patients were further classified into 3 groups. The first group received 1-2mg BMP-2 per level, the second group 2-3mg and the third group greater than $3 \mathrm{mg}$. The average fusion grades were 2.75 for group 1, 2.65 for group 2 and 2.83 for group 3. The average fusion grade was not statistically different between the groups.

Conclusions: The use of BMP-2 in MAS interbody fusion leads to high overall fusion rates. Furthermore, fusion success was not related to increasing the dose of BMP-2 and was reliably obtained even at lower doses. Using lower doses (1-2mg) of BMP-2 in MAS interbody fusions leads to reliable fusion rates while minimizing cost and potential complications.

\section{RF Paper \# 8. Morbidity and Mortality during Inpatient Admission for Osteoporosis-related Fractures}

Adam M. Lukasiewicz, MSc; Andre M. Samuel, BBA; Matthew L. Webb, AB; Daniel D. Bohl, MPH; Bryce A. Basques, BS; Jonathan Newman Grauer, $M D$

\section{Yale University School of Medicine}

Introduction: Spinal fractures are the most common type of osteoporosis-related fracture. However, the morbidity and mortal-

\begin{tabular}{|c|c|c|c|c|c|c|}
\hline & \multicolumn{2}{|c|}{ Mortality } & \multicolumn{2}{|c|}{ Any Adverse Event } & \multicolumn{2}{|l|}{ Length of stay } \\
\hline & $\mathrm{RR}$ & $p$ & RR & $p$ & Additional LOS (Days) & $p$ \\
\hline Male Sex & 1.30 & 0.025 & 0.74 & $<0.001$ & -0.05 & 0.336 \\
\hline \multicolumn{7}{|l|}{ Age } \\
\hline $18-65$ years & 0.45 & 0.003 & 0.72 & $<0.001$ & 0.37 & $<0.001$ \\
\hline $65-75$ years & 0.62 & 0.005 & 0.79 & $<0.001$ & -0.08 & 0.174 \\
\hline $75-80$ years & 0.71 & 0.027 & 0.89 & $<0.001$ & -0.04 & 0.47 \\
\hline \multicolumn{7}{|l|}{$80-85$ years } \\
\hline $85-90$ years & 1.14 & 0.002 & 1.05 & $<0.001$ & 0.02 & 0.176 \\
\hline $90+$ years & 1.48 & 0.295 & 1.10 & 0.013 & -0.08 & 0.719 \\
\hline Alcoholism & 1.07 & 0.861 & 1.24 & 0.001 & 0.62 & $<0.001$ \\
\hline Anemia & 1.35 & 0.003 & 1.20 & $<0.001$ & 1.05 & $<0.001$ \\
\hline Congestive heart failure & 2.35 & $<0.001$ & 1.32 & $<0.001$ & 1.19 & $<0.001$ \\
\hline Chronic pulmonary disease & 1.42 & $<0.001$ & 1.05 & 0.011 & 0.49 & $<0.001$ \\
\hline Coagulopathy & 1.86 & 0.002 & 1.23 & $<0.001$ & 1.04 & $<0.001$ \\
\hline Cerebrovascular Disease & 8.59 & $<0.001$ & 2.72 & $<0.001$ & 4.34 & $<0.001$ \\
\hline Dementia & 0.56 & 0.317 & 1.14 & 0.071 & 0.43 & 0.020 \\
\hline Depression & 0.73 & 0.028 & 1.06 & 0.011 & 0.21 & $<0.001$ \\
\hline Diabetes, uncomplicated & 0.77 & 0.06 & 1.14 & $<0.001$ & 0.21 & $<0.001$ \\
\hline Diabetes, with complications & 0.76 & 0.391 & 1.24 & $<0.001$ & 1.03 & $<0.001$ \\
\hline Drug abuse & 1.24 & 0.720 & 1.17 & 0.106 & 1.97 & $<0.001$ \\
\hline Electrolyte Abdormalities & 2.35 & $<0.001$ & 1.77 & $<0.001$ & 1.71 & $<0.001$ \\
\hline Hypertension & 0.92 & 0.362 & 0.98 & 0.218 & -0.01 & 0.889 \\
\hline Hypothyroid & 0.75 & 0.015 & 0.99 & 0.514 & -0.02 & 0.674 \\
\hline Obesity & 1.09 & 0.735 & 1.19 & $<0.001$ & 0.51 & $<0.001$ \\
\hline Peripheral Vascular Disease & 1.26 & 0.120 & 1.07 & 0.017 & 0.22 & 0.002 \\
\hline Renal Failure & 1.38 & 0.013 & 1.51 & $<0.001$ & 0.22 & 0.002 \\
\hline
\end{tabular}

ity associated with inpatient stays for osteoporosis-related spinal fractures are not known.

Methods: All patients with a primary diagnosis of spinal fracture and coexisting diagnosis of osteoporosis in the National Inpatient Sample (NIS) from 2005 to 2011 were identified. Patient with a diagnosis of malignant neoplasm and patients with supplemental diagnosis codes indicating major trauma were excluded. Descriptive statistics were used to characterize in-hospital adverse events, and multivariate Poisson regression was used to determine factors associated with adverse events. Multivariate linear regression was used to determine factors associated with increased length of stay.

Results: A total of 56,241 patients with spinal fractures and osteoporosis were identified. The average patient age was 80.3 years-old and $85.4 \%$ of patients were female. During in the inpatient period 515 patients $(0.9 \%)$ died. Overall, 12,669 patients $(22.5 \%)$ had a stay complicated by one or more adverse event. The most common adverse event was urinary tract infection, present in 9,011 patients $(16.0 \%)$, followed by acute kidney injury $(3.7 \%)$ and pneumonia $(3.3 \%)$. Other adverse events occurred in less than $1 \%$ of patients.

Patient factors predicting mortality, adverse events and length of stay are shown in Table 1 . The strongest predictors of mortality were cerebrovascular disease $(\mathrm{RR}=8.59)$, electrolyte abnormalities $(R R=2.35)$, and congestive heart failure $(R R=2.35)$. For mortality, male sex $(R R=0.74)$ and age less than $65(R R=0.72)$ were most protective, whereas cerebrovascular disease $(\mathrm{RR}=2.72)$ and electrolyte abnormalities $(\mathrm{RR}=1.77)$ had the greatest associated risk. For increased length of stay, most medical comorbidities had an effect, but the greatest magnitudes were for cerebrovascular disease (4.34 days) and drug abuse (1.97 days).

Discussion: Overall, approximately $1 \%$ of patients admitted for osteoporosis-related spinal fractures died during their inpatient admission. Almost a fifth of patients have a complicated inpatient course. Cerebrovascular disease and electrolyte abnormalities are particularly worrisome patient factors, predicting morbidity, mortality and length of stay.

Conclusion: Clinicians should be aware of the relatively high rate of complications in patients admitted with spinal fractures related to osteoporosis, particularly in patients with comorbid cerebrovascular disease and electrolyte disturbances.

RF Paper \# 9. The Effects Of Cage Size On Motor And Sensory Nerve Function During The Xlif® Procedure: Preliminary Results From A Prospective, Multi-center Study

Jim A. Youssef, MD; Juan S. Uribe, MD; Robert E. Isaacs, MD

\section{Durango Orthopedics}

Background: The availability of increasingly wide and increasingly tall PEEK implants are available for lateral lumbar interbody fusion. While a larger cage size has the advantage of indirect decompression through elevated disc height as well as added stability, insertion of a larger cage may require larger retraction of the psoas muscle and consequently a greater risk of injury to the surrounding tissue. The purpose of this study is to evaluate the effect of L4-5 cage size on postoperative changes in nerve function.

Methods: Patients from 21 sites who were undergoing XLIF at L4-5 were enrolled in a prospective, nonrandomized, multi-center, institutional review board (IRB)-approved, clinical study to evaluate the utility of triggered EMG throughout lateral retraction. As part of that research effort Intraoperative data collection included the interbody cage sizes and retraction size in the anterior/posterior and cranial/caudal directions. Pre-and postoperative 0-5 lower-extremity motor function were collected to identify new postoperative motor deficits.

Results: Three hundred eighteen (323) patients were enrolled. Postoperatively, 13 (4.0\%) patients had symptomatic motor neuropraxia on the approach side. The most prevalent cage dimensions were $22 \mathrm{~mm}$ width $(60.0 \%), 55 \mathrm{~mm}$ length $(45.3 \%)$, and $10 \mathrm{~mm}$ 
height (54.2\%).

Retractor clicks in the anterior-posterior direction were positively correlated with cage width $(\mathrm{p}<0.001)$, and clicks in the cranial-caudal direction were positively correlated with cage length $(p=0.005)$ and height $(p=0.007)$. Incidence of nerve injury, psoas weakness, post-op motor weakness distal to the psoas, and post-op sensory reduction were not significantly different across various cage dimensions.

Conclusions: The results of this study provide evidence that longer and wider interbody cages are predictors of larger retraction in the cranial-caudal direction, but not risk for increasing incidence of decreased motor or sensory function.

\section{RF Paper \# 10. Clinical Experience with Facet Arthroplasty: Two and Five Year Preliminary Outcomes from the ACADIA® IDE Study}

Jim A. Youssef, MD; Douglas Orndorff, MD; Rachel Ebner, MS, Sue Lynn Myhre, PhD

Spine Colorado

Introduction: An Investigational Device Exemption (IDE) study is being conducted to evaluate safety and efficacy of the ACADIA ${ }^{\circledR}$ Facet Replacement System.

Methods: Candidates have lateral, lateral recess and/or central canal stenosis at one level from L3 to S1 requiring decompression and facetectomy. Patients are randomized 2:1 to investigational ACADIA ${ }^{\circledR}$ or control PLF. The first two patients enrolled per site may be non-randomized to ACADIA ${ }^{\circledR}$. Outcome measures are collected pre-operatively and at 6 weeks, $3,6,12$ and 24 months and annually thereafter. Results are presented from 13 sites.

Results: 136 randomized patients completed 2 year follow-up (96 ACADIA $\AA$ and 40 PLF) and 26 completed 5 year follow-up (18 ACADIA ${ }^{\circledR}$ and 8 PLF). ACADIA ${ }^{\circledR}$ average ZCQ SS score improved from 3.6 pre-op to 1.9 at 2 years and 2.1 at 5 years, compared to 3.7 pre-op to 2.4 at 2 years and 2.3 at 5 years for PLF. ACADIA ${ }^{\circledR}$ ZCQ PF improved from 2.8 pre-op to 1.5 at 2 years and 1.6 at 5 years, while PLF scores improved from 2.8 pre-op to 1.9 at 2 years and 2.0 at 5 years. VAS back pain decreased from 67 pre-op to 19 at 2 years and 24 at 5 years for ACADIA $\AA$, and from 68 pre-op to 24 at 2 years and 38 at 5 years for PLF. VAS left leg pain decreased from 62 pre-op to 14 at 2 years and 16 at 5 years for ACADIA ${ }^{\circledR}$ versus 57 pre-op to 24 at 2 years and 13 at 5 years for PLF. VAS right leg pain improved from 55 pre-op to 13 at 2 years and 18 at 5 years for ACADIA $®$ and from 56 pre-op to 18 at 2 years and 15 at 5 years for PLF. Mean ODI improved for ACADIA ${ }^{\circledR}$ from 48 pre-op to 16 at 2 years and 21 at 5 years and from 49 pre-op to 26 at 2 years and 29 at 5 years for PLF.

Discussion: ACADIA® and PLF demonstrate sustained, longterm improvement in mean ZCQ, VAS and ODI scores.

Conclusion: Results suggest ACADIA ${ }^{\circledR}$ may be an effective treatment for lumbar spinal stenosis.
RF Paper\# 11.Establishing the Injury Severity of Thoracolumbar Trauma: Confirmation of the Hierarchical Structure of the AOSpine Thoracolumbar Spine Injury Classification System

Gregory D. Schroeder, MD; Alexander R. Vaccaro, MD, PhD; Christopher K. Kepler, MD, MBA; John D. Koerner, MD; Luiz Vialle, $M D$, PhD; Klaus J. Schnake, MD; Jens Chapman, MD; S. Rajasekaran, MD; Maximilian Reinhold, Priv.-Doz.Dr.; FC Oner, MD,PhD; Michael Fehlings, MD; Marcel Dvorak, MD; Frank Kandziora, MD, PhD

The Rothman Institute at Thomas Jefferson University

Introduction: The AOSpine Thoracolumbar Spine Injury Classification System was recently published and combines elements of both the Magerl system and the Thoracolumbar Injury Classification System (TLICS); however the injury severity of each fracture has yet to be established. The objective of this study was to develop a validated regional and global injury severity scoring system for thoracolumbar trauma.

Methods: A survey was sent to 100 AOSpine members from all six AO regions of the world (North America, South America, Europe, Africa, Asia and the Middle East). Each respondent was asked to numerically grade the severity of each variable of the AOSpine Thoracolumbar Spine Injury Classification System including the morphology, neurologic grade and patient specific modifiers. A grade of zero was considered to be not severe at all, and a grade of 100 was the most severe injury possible.

Results: Seventy-four AOSpine surgeons from all six AO regions of the world numerically graded the severity of each variable of the AOSpine Thoracolumbar Spine Injury Classification System to establish the injury severity score (Table 1$)$. The reported fracture severity increased significantly $(p<0.0001)$ as the subtypes of fracture Type A and Type B increased, and a significant difference $(\mathrm{p}<$ 0.0001 ) in severity was established for burst fractures with involvement of two versus one endplates. Lastly, no regional or experiential difference in severity or classification was identified.

Conclusion: Development of a globally applicable injury severity scoring system for thoracolumbar trauma is possible. This study demonstrates no regional or experiential difference in perceived severity or thoracolumbar spine trauma. The AOSpine Thoracolumbar Spine Injury Classification System provides a logical approach to assessing these injuries and enables rational strategies for treatment.

\begin{tabular}{|l|r|r|r|}
\hline \multicolumn{5}{|c|}{ Table 1 } \\
\hline Type & N & Mean & SD \\
\hline A0 & 74 & 5.09 & 5.07 \\
\hline A1 & 74 & 14.78 & 7.74 \\
\hline A2 & 74 & 29.81 & 14.41 \\
\hline A3 & 74 & 44.68 & 16.99 \\
\hline A4 & 74 & 59.7 & 18.77 \\
\hline B1 & 74 & 54.88 & 18.41 \\
\hline B2 & 74 & 69.09 & 17.66 \\
\hline B3 & 74 & 71.49 & 15.94 \\
\hline C & 74 & 94.8 & 10.18 \\
\hline N0 & 72 & 1.08 & 3.13 \\
\hline N1 & 72 & 19.19 & 17.14 \\
\hline N2 & 72 & 33.57 & 16.9 \\
\hline N3 & 72 & 79.79 & 19.07 \\
\hline N4 & 72 & 91.36 & 14.48 \\
\hline NX & 72 & 66.96 & 28.42 \\
\hline M1 & 72 & 50 & 23.67 \\
\hline M2 & 72 & 62.4 & 24.18 \\
\hline
\end{tabular}


RF Paper \# 12. A Worldwide Analysis of the Reliability and Perceived Importance of an Injury to the Posterior Ligamentous Complex in Thoracolumbar Trauma

Gregory D. Schroeder, MD; Christopher K. Kepler, MD, MBA; John Koerner, MD; FC Oner, MD,PhD; Marcel Dvorak, MD; Luiz R. Vialle, MD; Michael Fehlings, MD; Klaus Schnake, MD; Maximilian Reinhold, Priv.-Doz. Dr.; Jens R. Chapman, MD; S. RAJASEKARAN, MS(ORTH), MPhil, PhD; Frank Kandziora, MD, PhD; Alexander R. Vaccaro, $M D, P h D$

The Rothman Institute at Thomas Jefferson University

Summary of Background Data: The AOSpine Thoracolumbar Spine Injury Classification System was recently published and combines elements of both the Magerl system and the Thoracolumbar Injury Classification System (TLICS); however the accompanying treatment algorithm is still being developed. It is unclear how the M1 modifier, which indicates a significant ligamentous injury should affect the algorithm. The purpose of this study is to determine the worldwide reliability of spine surgeons indentifying a

\begin{tabular}{|l|r|}
\hline \multicolumn{2}{|c|}{ Table 1 } \\
\hline Region & Kappa Value \\
\hline Europe & 0.17 \\
\hline Asia Pacific & 0.08 \\
\hline Latin America & 0.10 \\
\hline Middle East & 0.07 \\
\hline $\begin{array}{l}\text { North } \\
\text { America }\end{array}$ & 0.22 \\
\hline Africa & 0.15 \\
\hline
\end{tabular}

posterior ligamentous complex (PLC) injury in a compression type fracture (A type), and to determine the perceived importance of the integrity of the PLC on the treatment algorithm.

Methods: A survey was sent to all AOSpine members from the six AO regions of the world. The survey consisted of ten cases of Type A fractures (2 subtype A1, 2 subtype A2, 3 subtype A3 and 3 subtype A4 fractures) with appropriate imaging (plain radiographs, $\mathrm{CT}$ and/or MRI), and the respondent was asked to identify fractures with a PLC disruption, as well as to indicate if the integrity of the PLC would affect their treatment recommendation.

Results: Five hundred and twenty nine spine surgeons from all six AO regions of the world completed the survey. The overall interobserver reliability in determining the integrity of the PLC was slight $(x=0.11)$. No substantial regional or experiential difference was identified (Table 1). A regional difference $(\mathrm{p}<0.001)$ in the importance of the PLC on the treatment of type A fractures was identified.

Conclusion: While the development of a globally applicable algorithm for thoracolumbar trauma is possible, with only slight interobserver agreement on the integrity of the PLC, the M1 modifier in the AOSpine Thoracolumbar Spine Injury Classification System cannot be a critical determinate of the recommended treatment.
RF Paper \# 13. Obesity Is Associated With An Increased Rate Of Incidental Durotomy In Lumbar Spine Surgery

Christopher A. Burks, MD, MS; Brian C. Werner, MD; Scott Yang, MD; Adam L. Shimer, M.D.

\section{University of Virginia}

Objectives: Incidental durotomy is a relatively common complication of lumbar spine surgery. There is a paucity of data on the overall impact of obesity on the rate of incidental durotomy in lumbar spine surgery specifically with regard to the type of procedure performed. The objective of this study was to utilize a large population database to determine the impact of obesity on the rate of incidental durotomy in specific lumbar spine procedures.

Methods: Patients who underwent lumbar spine surgery were identified using the PearlDiver Patient Records Database (www. pearldiverinc.com) using the Current Procedural Terminology (CPT) codes for single and multi-level hemilaminotomy, laminectomy, fusion with or without decompression, and revision decompressions. They were then stratified into separate cohorts based on ICD-9 codes for obesity, as measured by body mass index (BMI), and by CPT codes for specific lumbar spine procedures. Documentation of incidental durotomy was noted utilizing the ICD-9 codes for durotomy (349.31 and 349.39). Patient demographics and associated comorbidities were assessed. Odds ratios and 95\% confidence intervals were calculated and chi square test was used to assess for statistical significance [Table 1].

Results: The rate of incidental durotomy ranged from 0.5-2.6\% with the highest rates observed in multi-level laminectomies and revision decompressions in the obese and morbidly obese groups. For patients who underwent decompression only procedures, nonobese patients had a significantly lower rate of durotomy than the obese and morbidly obese cohorts $(0.7 \%$ vs $0.9 \%$ vs $1.2 \%$ respectively, $\mathrm{p}<0.5$ ). For patients who underwent fusion with or without decompression, there was a significantly increased rate of durotomy in obese patients compared to non-obese patients ( $1.3 \%$ vs $1.6 \%, \mathrm{p}$ $<0.5)$. The morbidly obese cohort also had significantly higher rates of incidental durotomy than the non-obese cohort in both revision decompression and revision fusion procedures.

Conclusions: This analysis of a large administrative database demonstrates that obesity is associated with increased rates of incidental durotomy in lumbar spine surgery. Furthermore, obesity, in association with increasing complexity of the procedure, increases the rate of incidental durotomy in lumbar spine surgery. Surgeons must be aware of these increased risks as the rate of obesity increases in the population.

\begin{tabular}{|c|c|c|c|c|c|c|}
\hline & \multicolumn{2}{|c|}{ BMI $>40$ vs $<30$} & \multicolumn{2}{|c|}{ BMI 30 to 40 vs $<30$} & \multicolumn{2}{|c|}{ BMI 30 to 40 vs $>40$} \\
\hline & $O . R$. & $P$ & $O . R$. & $P$ & $O . R$ & $P$ \\
\hline \multicolumn{7}{|l|}{ Decompression Alone } \\
\hline Decompression Overall & 1.75 & $<0.0001$ & 1.34 & $<0.0001$ & 1.30 & 0.02 \\
\hline 1 Level HemiLaminotomy & 1.74 & 0.001 & 1.36 & 0.016 & 1.28 & 0.239 \\
\hline 1 Level Laminectomy & 1.69 & 0.038 & 0.98 & 1.00 & 1.71 & 0.09 \\
\hline $2+$ Level HemiLaminotomy & 1.05 & 0.967 & 1.04 & 0.967 & 1.01 & 0.862 \\
\hline $2+$ Level Laminectomy & 1.81 & $<0.0001$ & 1.34 & 0.009 & 1.35 & 0.085 \\
\hline \multicolumn{7}{|l|}{ Fusion +/- Decompression } \\
\hline Posterior Fusion & 1.25 & 0.074 & 1.26 & 0.018 & 1.00 & 0.95 \\
\hline \multicolumn{7}{|l|}{ Revision } \\
\hline Revision Decompression & 1.59 & 0.04 & 1.47 & 0.02 & 1.08 & 0.859 \\
\hline Revision Fusion & 1.77 & 0.03 & 1.15 & 0.615 & 1.54 & 0.215 \\
\hline All Spine Surgeries & 1.54 & $<0.0001$ & 1.33 & $<0.0001$ & 1.16 & 0.085 \\
\hline
\end{tabular}




\section{RF Paper \# 14. Complex Combat-Related Lumbosacral Dissociations}

Scott Wagner, MD; Gregory Van Blarcum, MD; Peter M. Formby, MD; Daniel G. Kang, MD; Ronald A. Lehman Jr., MD

\section{Walter Reed National Military Medical Center}

Introduction: As war injury patterns have changed throughout Operations Iraqi and Enduring Freedom (OIF/OEF), a relative increase in the incidence of complex lumbosacral dissociation (LSD) injuries has been noted. LSD injuries are an anatomic separation of the spinal column from the pelvis, and represent a manifestation of severe, high energy trauma. We assessed the clinical outcomes of combat-related LSD injuries after operative treatment.

Methods: We performed a retrospective review of records and radiographs for all patients surgically managed for complex LSD injuries at one institution since the beginning of current combat operations in the Middle East.

Results: Twenty patients met inclusion criteria and were treated as follows: posterior spinal fusion (12), sacroiliac screw fixation (7), and combined anterior-posterior fusion (1). The mean age was 28.2 years old. The most common mechanism of injury was mounted improvised explosive device (IED, 50\%). On average, 2.2 spinal regions were injured per patient. Neurologic dysfunction was present in four patients. Three patients underwent operative stabilization of their injuries before evacuation to the United States. Median time to surgery from injury was 12 days (range: 0-111 days). There was a $20 \%$ wound infection rate.

Median follow up was 45.5 months (range: 23.2-105.3 months), and one-third of all patients were medically retired due to their injuries. At most recent follow up, $45 \%$ complained of chronic low back pain and 35\% had persistent neurologic deficits; however, $30 \%$ were actively engaged in strenuous physical activities, including swimming, distance running and training for Special Forces.

Discussion: This is the largest series of operatively managed lumbosacral dissociation patients currently reported. Our series suggests that combat-related LSD injuries frequently result in persistent, long-term neurologic dysfunction, disability and chronic pain. Operative management carries a high post-operative risk of infection. However, two-thirds of patients were able to remain on active duty after their injuries, and two patients were able to complete marathon training.

Conclusion: Though fraught with complications, including a high post-operative infection rate, chronic pain and persistent neurologic dysfunction, operative treatment of combat-related lumbosacral dissociation injuries can yield good functional results at four years in a relatively high percentage of patients.

\section{RF Paper \# 15. Percutaneous Pedicle Screw Accuracy Using a Primarily AP Fluoroscopy Technique in Trauma Patients}

Seth K. Williams, MD

University of Wisconsin Dept of Orthopedics and Rehabilitation

Introduction: Thoracic and lumbar spine fractures in neurologically intact patients can usually be managed non-operatively with bracing, but some fractures are unstable and are best treated surgically. We aim to assess the accuracy of percutaneous pedicle screw instrumentation using predominantly anteroposterior AP fluoroscopy.

Methods: Between September 2008 and April 2013, 35 neurologically intact patients with thoracolumbar fractures underwent percutaneous posterior pedicle screw fixation by a single surgeon. Surgical indications were unstable fractures as evidenced by traumatic kyphosis that was progressive with attempted brace treatment, 3-column injury involving disruption of the posterior ligamentous complex, and displaced fractures in an ankylosed spine. Multitrauma precluding brace treatment was a less common relative indication. Retrospective review of imaging studies was performed. Pedicle cannulation was performed with predominantly AP fluoroscopy, switching to lateral fluoroscopy only once the Jamshidi needle had passed through the pedicle and entered the vertebral body.

Results: Eleven lower lumbar (L2 - L5), 12 thoracolumbar (T12 and L1), and 12 thoracic level fractures were treated with a total of 248 pedicle screws. The screws were placed percutaneously under fluoroscopic guidance with blunt dissection through the paraspinal musculature. Postoperative CT scans $(\mathrm{n}=28)$ showed accurate intrapedicular placement in 183 of 188 pedicles screws $(97.3 \%)$. All 5 pedicle breaches were asymptomatic and all occurred in ankylosed thoracic spines with extension fractures. One breach was $2 \mathrm{~mm}$ medial, and the other 4 were less than $3 \mathrm{~mm}$ lateral with the screw subsequently entering the vertebral body as occurs in an "in-out-in" thoracic pedicle screw technique. No screws required revision.

Conclusion: Percutaneous pedicle screw fixation was accomplished with a high degree of accuracy using AP fluoroscopy to guide pedicle cannulation. All breaches occurred in patients with ankylosed spines, who often have difficult fluoroscopic anatomy. Nevertheless, these breaches were minimal and inconsequential. Percutaneous pedicle screw fixation can be safely performed using predominantly AP fluoroscopy.

RF Paper \# 16. Occult Pathogens: Are they the missing link to Failed Back Syndrome?

Darren R. Lebl, MD; Tucker C. Callanan, BS; William Long, MD; Byung Jo Victor Yoon, MS; Celeste Abjornson, PhD

Hospital for Special Surgery

Introduction: In as high as $40 \%$, patients continue to experience chronic pain after spine surgery even when surgical parameters such as fusion status and hardware placement are normal. Occult spinal infections provide a differential diagnoses to be considered in this condition. This study explored the prevalence of occult infections in patients who were undergoing revision spine surgery who had persistent, disabling pain of no known origin.

Methods: Patients undergoing revision spinal surgery requiring hardware removal were consent to participate. Antibiotics were held prior to surgery. At time of hardware removal, periprosthetic tissue surrounding each screw hole of each implant was collected for gram staining, histopathological analysis and long cultures in order to identify tissue changes that were suggestive of chronic infection. The removed hardware was then scanned with an environmental Scanning Electron Microscope (eSEM) to examine the instrumentation for changes on the surface of the implant that may assist in the harboring of bacteria. The results of the implant were blinded from the surgeon and the imager.

Results: Occult adherent bacteria were found in $31 \%$ of the tissue surrounding the implants of patients undergoing spine revision surgery. Bacteria found in the tissue surrounding the removed implants included Propionibacterium acnes, Klebsiela pneumoniae, Staphylococcus simulans, Staphylococcus epidermidis, as well as Staphylococcus capitis, and its subspecies (ureolyticus and capitis). Etching and pitting consistent with normal wear and removal was visible on each implant analyzed via SEM but not abnormal to revision surgeries.

Discussion: Chronic pain in patients who have undergone spine surgery severely affects the patient's quality of life leading to a significant impact on the health care system. As the rates of chronic pain after spine surgery continue to rise, identifying the factors that contribute to its development are critical in better managing and preventing this disabling condition. The results of this study demonstrate that occult infections could be a contributing factor to this issue.

Conclusion: The findings of this study have significant clinical 
impact indicating that occult infections are far more prevalent in patients requiring revision surgery than previously identified.

\section{RF Paper \# 17. The Utility of Obtaining Routine Hematologic Laboratory Values Following a Minimally Invasive Transforaminal Lumbar Interbody Fusion}

Junyoung Ahn, BS; Blaine T. Manning, BS; Andrew Park, BS; Vincent Rossi, BS, BA; Islam Elboghdady, BS; Kern Singh, MD

\section{Rush University Medical Center}

Introduction: Minimally invasive (MIS) transforaminal lumbar interbody fusion (TLIF) is associated with decreased blood loss and morbidity; however, many institutions obtain routine post-operative hematologic values including a complete blood count and basic metabolic panel. The purpose of this study is to determine the utility of obtaining routine post-operative hematologic laboratory values following an MIS-TLIF.

Methods: A retrospective analysis of 334 patients who underwent a primary, single-level MIS-TLIF for degenerative lumbar spine pathology between 2007-2013 was performed utilizing a prospectively maintained registry. Demographics, comorbidity (Charlson Comorbidity Index, CCI), smoking status, pre- and post-operative Visual Analogue Scores (VAS), procedural time, estimated blood

\begin{tabular}{|c|c|c|c|c|c|c|c|c|c|}
\hline & \multicolumn{2}{|c|}{ All Cohorts } & \multicolumn{4}{|c|}{ Unilateral Instrumentation Cohort } & \multicolumn{3}{|c|}{ Bilateral Instrumentation Cohort } \\
\hline & Pre-operative & Post-operative & $\mathrm{p}$-value & Pre-operative & Post-operative & $\mathrm{p}$-value & Pre-operative & Post-operative & $\mathrm{p}$-value \\
\hline $\mathrm{Hb}$ & $14.4 \pm 1.5$ & $12.5 \pm 1.5$ & $<0.001$ & $14.3 \pm 1.6$ & $12.5 \pm 1.5$ & $<0.001$ & $14.5 \pm 1.5$ & $12.5 \pm 1.5$ & $<0.001$ \\
\hline $\mathrm{Ht}$ & $42.4 \pm 4.0$ & $37.4 \pm 4.0$ & $<0.001$ & $42.1 \pm 4.0$ & $37.7 \pm 3.9$ & $<0.001$ & $42.7 \pm 4.0$ & $37.2 \pm 4.2$ & $<0.001$ \\
\hline Glucose & $99.1 \pm 25.7$ & $118.8 \pm 33.4$ & $<0.001$ & $98.6 \pm 24.1$ & $116.0 \pm 31.1$ & $<0.001$ & $99.7 \pm 27.6$ & $122.4 \pm 35.9$ & $<0.001$ \\
\hline BUN & $14.6 \pm 5.2$ & $10.9 \pm 3.8$ & $<0.001$ & $14.4 \pm 5.8$ & $10.9 \pm 3.8$ & $<0.001$ & $14.9 \pm 4.5$ & $11.0 \pm 3.7$ & $<0.001$ \\
\hline $\mathrm{Cr}$ & $0.92 \pm 0.19$ & $0.87 \pm 0.18$ & $<0.001$ & $0.91 \pm 0.18$ & $0.87 \pm 0.19$ & $<0.001$ & $0.92+0.2$ & $0.88 \pm 0.19$ & 0.002 \\
\hline $\mathrm{Na}^{+}$ & $139.4 \pm 2.7$ & $137.4 \pm 2.5$ & $<0.001$ & $139.0 \pm 2.8$ & $136.9 \pm 2.5$ & $<0.001$ & $139.6 \pm 2.4$ & $138.2 \pm 2.3$ & $<0.001$ \\
\hline $\mathrm{K}^{+}$ & $4.3 \pm 0.38$ & 4.1 $1 \pm 0.33$ & $<0.001$ & $4.3 \pm 0.39$ & 4.1 $1 \pm 0.35$ & $<0.001$ & $4.25 \pm 0.36$ & $4.07 \pm 0.31$ & $<0.001$ \\
\hline $\mathrm{Cl}-$ & $104.2+2.9$ & $104.7 \pm 2.7$ & 0.02 & $104.4+2.9$ & $104.5 \pm 2.6$ & 0.659 & $104.0 \pm 2.9$ & $104.5 \pm 3.4$ & 0.003 \\
\hline $\mathrm{Ca}^{++}$ & $9.5 \pm 0.52$ & $8.5 \pm 0.47$ & $<0.001$ & $9.5 \pm 0.49$ & $8.5 \pm 0.46$ & $<0.001$ & $9.6 \pm 0.55$ & $8.5 \pm 0.5$ & $<0.001$ \\
\hline
\end{tabular}

loss (EBL), length of hospitalization (LOS), complication rates, transfusion volumes, and electrolytes replacement were assessed. The patient's post-operative hemoglobin, hematocrit, glucose, blood urea nitrogen (BUN), creatinine, sodium, potassium, chloride, and calcium were compared to pre-operative values. Statistical analysis was performed utilizing Paired-Sample T-tests.

Results: A total of 334 single-level MIS-TLIF procedures were identified. The mean age of the cohort was $50.4 \pm 12.6$ years. The overall mean procedural time, EBL, and LOS were $123.3 \pm 39.6$ minutes, $79.06 \pm 82.6$ cc, and $64.8 \pm 29.9$ hours, respectively. Following a MIS-TLIF, the post-operative hemoglobin, hematocrit, BUN, creatinine, sodium, potassium, and calcium statistically decreased while glucose increased when compared to the preoperative values $(p<0.05)$. None of the patients in both cohorts required intra-operative or post-operative blood product transfusion. Fifteen (4.5\%) patients required post-operative potassium replacement for clinically asymptomatic laboratory values.

Discussion: The majority of MISTLIF patients did not require any action to be taken based upon the patient's routine post-operative hematologic laboratory data. Despite significantly lower post-operative hemoglobin and hematocrit levels compared to pre-operative values, none of the patients required blood product transfusions.

Conclusions: The findings of this analysis suggest that obtaining routine post-operative complete blood counts or basic metabolic panels following an uncomplicated MIS TLIF may have limited clinical value unless significant intra-operative bleeding is noted or the patient carries risk factors for coagulopathies, cardiac disease, renal dysfunction or post-operative anemia.

\section{RF Paper \# 18. Rationale for the Surgical Treatment of Lumbar} Degenerative Spondylolisthesis

Gregory D. Schroeder, MD; Christopher K. Kepler, MD, MBA; Mark Kurd, MD; Alexander R. Vaccaro, MD, PhD; Alpesh A. Patel, $M D$; Wellington K. Hsu, MD; Jason W. Savage, MD

The Rothman Institute at Thomas Jefferson University

Background: The classic treatment for patients with symptomatic degenerative spondylolisthesis is decompression and fusion, however some patients may benefit from an isolated decompression. The purpose of this study is to determine the effect of patient age, the presence of instability and/or the presence of low back pain on the treatment of patients with a degenerative spondylolisthesis, and to determine if treatment strategy is affected by surgeon specialty, location or practice model.

Methods: A survey was sent to surgeon members of the Lumbar Spine Research Society and AOSpine requesting information regarding their preferred treatment of degenerative spondylolisthesis. Determinants included patient age, the presence of instability, the presence of low back pain, surgeon's location, surgeon's specialty, and practice model.

Results: Two hundred and twenty three spine surgeons completed the survey. Age of the patient, the presence of instability, and the presence of low back pain all significantly $(\mathrm{p}<0.0001)$ affected the recommended treatment (Table 1). These were independent of surgeon factors. Older patients were significantly less likely to undergo an interbody fusion and more likely to undergo an isolated decompression $(p<0.0001)$, and the presence of instability made either an interbody fusion or a posterolateral fusion more likely than an isolated decompression $(\mathrm{p}<0.0001) .53 .2 \%$ of surgeons reported they would recommend an isolated decompression for a properly selected patient with a degenerative spondylolisthesis.

Conclusions: The gold standard treatment for a degenerative spondylolisthesis at this point in time is a decompression and fusion, but surgeons believe degenerative spondylolisthesis is a heteroge-

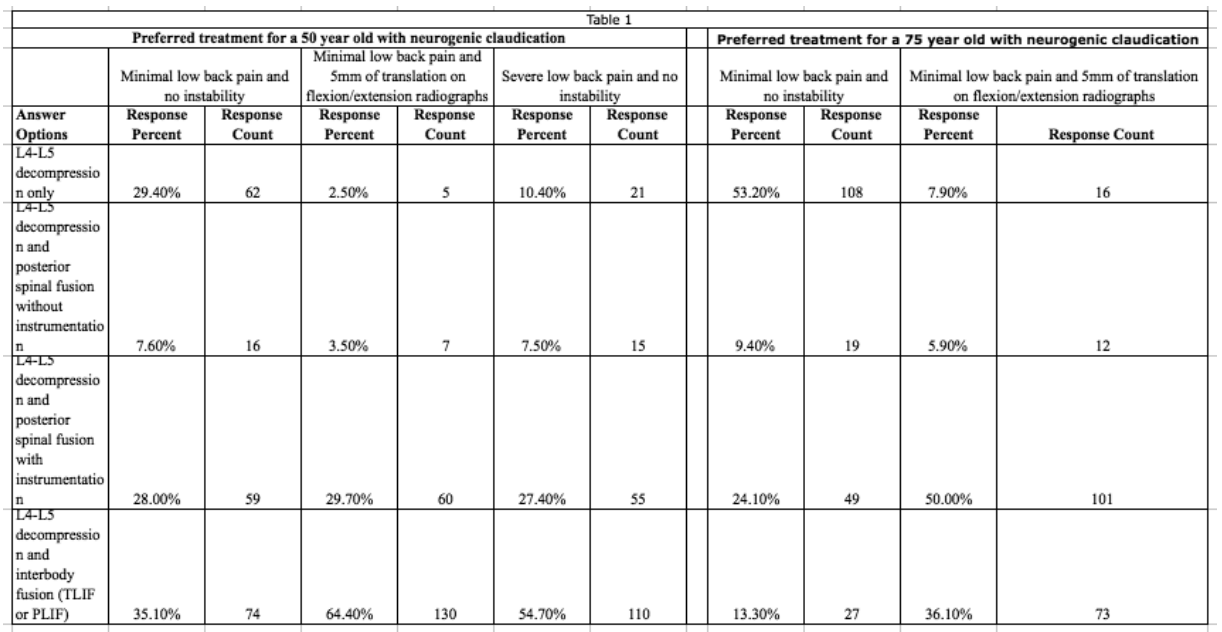


neous pathology that requires an individualized surgical plan. Future studies are needed to evaluate the effect of variables such as age, the presence of low back pain and the presence of instability on patient reported outcomes from various surgical options.

RF Paper \# 19. Minimally Invasive, Stereotactic, Wireless, Percutaneous Pedicle Screw Placement in the Lumbar Spine: Accuracy Rates with 224 Consecutive Screws

Blake N. Staub, MD; Virendra Desai, MD; Paul J. Holman, MD

Houston Methodist Neurological Institute

Introduction: Standard fluoroscopic and stereotactic CT-guided lumbar pedicle screw instrumentation has traditionally relied on the placement of Kirshner wires (K-wires) to ensure accurate screw placement. The use of $\mathrm{K}$-wires, however, is associated with a known risk of morbidity due to potential ventral displacement into the retroperitoneum. We report our cumulative experience using a computer, image-guided, wireless method for pedicle screw placement. We hypothesize that minimally invasive, wireless pedicle screw placement is as accurate and safe as the traditional technique utilizing $\mathrm{K}$-wires, while decreasing operative time and avoiding the potential morbidity associated with K-wires.

Methods: A retrospective review was undertaken of the first 48 consecutive patients in which we utilized a stereotactically-guided, wireless technique to place lumbar pedicle screws. All the constructs were supplemented by anterior or anterolateral interbody cage placement. The cases were performed using the O-arm and stereotactic instrumentation. After placing a percutaneous, navigation frame into the ilium, pilot holes were drilled with an extra long match stick burr within a navigated universal drill guide. The trajectory plan was then saved on the Stealth Station prior to removal of the drill bit. Rather than inserting a K-wire at this point, the drill and drill guide were removed. Using the saved plan, the pilot hole was easily palpated and subsequently tapped with an image guided awl tip tap. The screws were then inserted using a navigated screw driver and the saved trajectory plan as a tactile and visual guide. An O-arm scan was then done to confirm screw placement.

Results: There were 24 females and 24 males with an average age of 56.224 pedicle screws were placed using the stereotactic, wireless technique. Only 2 screws were inaccurately placed and required repositioning. The overall accuracy was $99.1 \%$. The two misplaced screws occurred in the same patient, were equally low, and were replaced with no increased patient morbidity.

Conclusion: Wireless, percutaneous placement of lumbar pedicle screws utilizing CT-guided stereotactic navigation appears safe with extremely high accuracy rates of greater than $99 \%$.

RF Paper \#20. A Cost-Effectiveness Comparison Between Transforaminal and Lateral Lumbar Interbody Fusions Using the Incremental Cost-Effectiveness Ratio at 2 Year

Gurpreet S. Gandhoke, MD; Han-Moe Shin, NA; Yue-Fang Chang, PhD; Zachary Tempel, MS; Peter C. Gerszten, MD; David O. Okonkwo, MD, PhD; Adam S. Kanter, MD

\section{University Of Pittsburgh Medical Center}

Introduction: Both transforaminal lumbar interbody fusion (TLIF) and lateral lumbar interbody fusion (LLIF) are effective surgical interventions for appropriately selected patients with degenerative lumbar spondylosis. This study sought to compare health care costs associated with these procedures by calculating the incremental cost-effectiveness ratio (ICER) and, thereby, the difference in the total cost per quality adjusted life year (QALY) gained for TLIF versus LLIF for the treatment of degenerative spondylosis. We further calculated the thresholds for Minimum Clinically Important Difference and Minimum Cost Effective Difference for patientreported outcome measures at 2-year follow-up.

Methods: Forty-five patients who underwent single level TLIF and 29 patients who underwent single level stand-alone LLIF for degenerative spondylosis with low back and leg pain were included. All costs from diagnosis through 2-year postsurgical follow up were available from a comprehensive single center data bank within a unified hospital system. Total cost to the third-party payor for all spine-related medical resource use from the time of diagnosis through 2 years was recorded. QALYs were calculated from EQ5D collected in an unbiased manner by a non-clinical staff member. Difference in total cost per QALY gained for LLIF minus that for TLIF was assessed as the incremental cost-effectiveness ratio ICER: (Cost LLIF- Cost TLIF)/(QALY LLIF- QALY TLIF).

Results: Significant improvements were observed at 2 year follow up for both TLIF and LLIF utilizing SF36PCS, ODI, VAS BP, VAS LP and EQ5D. ICER calculations revealed similar mean cumulative QALYs gained at the 2-year interval ( 0.67 for TLIF and 0.60 for LLIF; $\mathrm{p}=0.331)$. Median total cost of care following TLIF and LLIF were $\$ 44,068$ and $\$ 45,574$, respectively; $(p=0.960)$. MCED thresholds with an anchor of $<\$ 50,000 / \mathrm{QALY}$ were higher than MCID thresholds (calculated using the Health Transition Index anchor) for all patient-reported outcome measures. Total mean cost and EQ5D were statistically equivalent between the 2 treatment groups.

Conclusions: Transforaminal lumbar interbody fusion (TLIF) and lateral lumbar interbody fusion (LLIF) produced equivalent 2-year patient outcomes at an equivalent cost effectiveness profile. 


\section{Poster \# 1. Vertebral Body Biopsy During Vertebral Augmentation. Is It Indicated?}

Rasheed Abiola, MD; Paul A. Anderson, MD

University of Wisconsin Hospital and Clinics

Introduction: Vertebral body biopsy is possible during vertebral augmentation for treatment of painful osteoporotic fractures, but no standards are established to determine indication. To differentiate pathologic fractures from neoplasm or osteoporotic in patients with history of neoplasm is difficult and biopsy may be warranted. The purpose of this study is to determine if vertebral body biopsy is necessary in all patients or a subset undergoing vertebral augmentation.

Methods: Investigators performed a systematic review for which nine studies met the criteria with a total of 1398 patients. Subjects were divided into two groups: low-risk and high-risk patients. The low-risk patients are without history of neoplasm and no suspicious findings of neoplasm on imaging. The high-risk patients have a history of neoplasm or those having suspicious findings on imaging. Random effect model was used in the meta-analysis looking at positive biopsy in both low and high-risk groups. Funnel plot was also done to assess for publication bias.

Results: Nine studies were identified including a total of 1398 patients. The low-risk patients had an incidence of positive biopsies of $2.7 \%$ ( $95 \%$ CI: 0.71 to $9.3 \%$ ). The heterogeneity was large with I square values of $89 \%$. Single elimination of one study with a high incidence lowered the pooled incidence to $1.8 \%$ (95\% CI: 0.86 to $3.8 \%$ ). In the high-risk group, the incidence of positive biopsies was $29 \%$ (95\% CI: 10.9 to $58.5 \%$ ) The heterogeneity was again I squared of $75.9 \%$. There was no publication bias. No adverse events were reported related to the biopsy. Only one case of potential false negative was reported.

Discussion: Vertebral body biopsy is an accurate way of diagnosing tumor in insufficiency fractures. The meta-analysis provides evidence in favor of vertebral body biopsy in low risk patient at the time of vertebral augmentation. Patients who are at high risk should routinely have biopsy when undergoing vertebral augmentation.

Conclusion: Incidence of positive biopsy in high-risk and lowrisk patients are $29 \%$ and $2.7 \%$ respectively. This evidence is in favor of biopsy in low-risk patients and supports routine biopsy in high-risk patients.

\section{Poster \# 2. Interbody Cage Height Affects Subsidence Rate After Transforaminal Lumbar Interbody Fusion}

data, including number of levels fused, implant material/length/ height, and use of bone morphogenetic protein (BMP).

Results: We identified 128 patients with complete CT imaging. Average follow up was 27.2 months and overall fusion rate was $56 \%$. Fifty-five $(43.0 \%)$ had evidence of implant subsidence at most recent follow up, with average erosion into the superior endplate of the inferior vertebral body of 5.5 millimeters. The remaining 73 patients had no implant subsidence. There were no demographic differences between groups. The most commonly used interbody cage material was polyetheretherketone (PEEK). The subsidence group had a higher average number of levels fused (2 vs 1.7, $\mathrm{p}=0.05$ ). There was no difference in BMP use or implant surface area between groups. Implant height was significantly higher in the subsidence group $(12.6 \mathrm{~mm})$ when compared to the no-subsidence group (11.2mm, $\mathrm{p}<0.0001)$.

Discussion: Our study found higher number of fusion levels and an increased interbody cage height of only $1.4 \mathrm{~mm}$ were significantly associated with the occurrence of interbody cage subsidence after TLIF, with an average cage subsidence of more than half a centimeter. This finding may be related to intensive endplate preparation to fit a larger cage, or due to the cage itself. The clinical relevance of increased fusion levels is unclear.

Conclusion: Our data suggest that a larger height interbody cage may ultimately lead to violation of the endplate over time. The risk of interspace collapse, as well as global lumbar height loss, must be considered during intraoperative implant height and size selection.

\section{Poster \# 3. Short-term Outcomes After Cauda Equina Syndrome: An Analysis Of 3,732 Patients}

Andre M. Samuel, BBA; Adam M. Lukasiewicz, MSc; Matthew L. Webb, AB; Daniel D. Bohl, MPH; Bryce A. Basques, BS; Jonathan Newman Grauer, MD

\section{Yale School of Medicine}

Introduction: Cauda equina syndrome is an emergent spinal cord injury that requires rapid surgical treatment. As it is relatively uncommon, there remain no large case series considering peri-operative outcomes during the initial hospitalization of these patients.

Methods: All patients with cauda equina syndrome in the 2011 National Inpatient Sample (NIS) were identified. Rates of in-hospital adverse events and mortality were determined and multivariate regression was used to identify factors associated with adverse events, increased mortality, and longer length of stay.
Scott Wagner, MD; Peter M. Formby, MD; Daniel G. Kang, MD; Melvin Helgeson, MD

\section{Walter Reed National Military Medical Center}

Introduction: Various finite element and biomechanical studies have examined the relationship between implant positioning, preparation of the vertebral endplate and techniques of posterior fixation to construct stability after transforaminal lumbar interbody fusion (TLIF). A larger interbody cage footprint placed at the apohyseal ring is postulated to minimize implant subsidence after insertion. We set out to analyze interbody cage characteristics and risk factors for subsidence after TLIF.

Methods: We performed retrospective review of patients over age 50 undergoing TLIF at a single institution over a ten year period. Computed tomography (CT) scans, plain radiographs and medical records were reviewed for patient demographic information and surgical

\begin{tabular}{|c|c|c|c|c|c|c|}
\hline \multirow[b]{2}{*}{$\begin{array}{l}\text { Dependent variable: Mortality } \\
\mathrm{n}=3,728\end{array}$} & \multicolumn{2}{|c|}{ Adverse events } & \multicolumn{2}{|r|}{ Mortality } & \multicolumn{2}{|c|}{ Length of stay } \\
\hline & $\begin{array}{l}\text { Adverse } \\
\text { event rate }\end{array}$ & $\begin{array}{l}\text { Multivariate odds } \\
\text { ratio }\end{array}$ & $\begin{array}{l}\text { Mortality } \\
\text { rate }\end{array}$ & $\begin{array}{l}\text { Multivariate odds } \\
\text { ratio }\end{array}$ & $\begin{array}{l}\text { Mean length of } \\
\text { stay (days) }\end{array}$ & $\begin{array}{c}\text { Multivariate } \\
\text { regression } \\
\text { coefficient (davs) }\end{array}$ \\
\hline \multicolumn{7}{|l|}{ Age } \\
\hline $18-59$ years & $29.1 \%$ & $0.83(0.69-1.00)$ & $1.1 \%$ & $0.66(0.32-1.36)$ & 7.4 & $-0.49(-1.27-0.29)$ \\
\hline $60-69$ years & $33.6 \%$ & reference & $1.6 \%$ & reference & 8.0 & reference \\
\hline $70-79$ years & $38.9 \%$ & $1.23(0.98-1.53)$ & $2.4 \%$ & $1.38(0.65-2.96)$ & 7.8 & $-0.32(-1.28-0.64)$ \\
\hline $80+$ years & $43.2 \%$ & $1.45(1.12-1.87)$ & $4.2 \%$ & $2.37(1.12-5.02)$ & 6.5 & $-1.72(-2.82--0.61)$ \\
\hline Alcoholism & $35.6 \%$ & $0.99(0.65-1.53)$ & $3.0 \%$ & $1.97(0.58-6.66)$ & 10.3 & $1.78(-0.06-3.63)$ \\
\hline Congestive heart failure & $62.2 \%$ & $2.88(2.12-3.93)$ & $5.6 \%$ & $2.75(1.35-5.60)$ & 9.7 & $2.09(0.72-3.46)$ \\
\hline Chronic pulmonary disease & $39.8 \%$ & $1.22(1.01-1.48)$ & $3.0 \%$ & $1.76(0.99-3.13)$ & 7.8 & $0.06(-0.77-0.89)$ \\
\hline Coagulopathy & $54.3 \%$ & $2.45(1.84-3.27)$ & $5.3 \%$ & $3.45(1.76-6.78)$ & 12.0 & $4.57(3.27-5.87)$ \\
\hline Diabetes, uncomplicated & $36.7 \%$ & $1.16(0.96-1.41)$ & $1.5 \%$ & $0.90(0.44-1.83)$ & 7.7 & $027(-0.54-1.08)$ \\
\hline Diabetes, with complications & $47.2 \%$ & $1.67(1.21-2.30)$ & $2.3 \%$ & $1.15(0.39-3.36)$ & 9.8 & $2.29(0.88-3.71)$ \\
\hline Drug abuse & $43.2 \%$ & $1.77(1.23-2.56)$ & $0.8 \%$ & $0.44(0.06-3.29)$ & 11.0 & $3.23(1.61-4.85)$ \\
\hline Hypertension & $33.8 \%$ & $0.86(0.74-1.00)$ & $1.5 \%$ & $0.54(0.31-0.92)$ & 7.6 & $0.00(-0.63-0.64)$ \\
\hline Obesity & $33.3 \%$ & $1.05(0.86-1.28)$ & $0.7 \%$ & $0.39(0.14-1.09)$ & 7.0 & $-0.78(-1.60-0.03)$ \\
\hline
\end{tabular}

Note: Statistically significant results are highlighted in bold. False discovery rate control was utilized to determine the threshold for statistical significance in each analysis.

Adverse events include death, sepsis, myocardial infarction, cerebrovascular accident, thromboembolic events, acute kidney injury, pneumonia, urinary tract infection, and surgical site infection.

$\mathrm{SD}=$ standard deviation 
Results: A total of 3,732 patients with cauda equine syndrome were identified. Median age was 59 years. Mean length of stay was 7.7 days. The most common in-hospital adverse events were urinary tract infection $(22.0 \%)$, acute kidney injury (7.6\%), and sepsis $(6.9 \%)$, while the overall rate of any adverse events was $33.2 \%$. Mortality rate was $1.8 \%$ (66 patients).

Table 1 shows patient risk factors for adverse events, mortality, and longer length of stay. The risk factors with the strongest associations with adverse events were congestive heart failure $(\mathrm{OR}=2.88)$ and coagulopathy $(\mathrm{OR}=2.45)$, with the adverse event rate rising to $62.2 \%$ and $54.3 \%$ in these subpopulations, respectively. Mortality was associated with coagulopathy $(\mathrm{OR}=3.45)$ and congestive heart failure $(\mathrm{OR}=2.75)$, with the mortality rate rising to $5.3 \%$ and $5.6 \%$ in these subpopulations, respectively.

Discussion: The overall rate of adverse events is high in these patients, at $33.2 \%$, and increased to over $50 \%$ in certain subpopulations with preexisting comorbidities. This highlights the need for aggressive medical management of these patients during the initial hospitalization. As would be expected, mortality is low after cauda equina injuries, however preexisting congestive heart failure and coagulopathy are also associated with increases in mortality.

Conclusion: The overall rate of in-hospital adverse events is high after cauda equina syndrome and correlates with preexisting medical comorbidities.

Poster \# 4. Minimally Invasive Transforaminal Lumbar Interbody Fusion: Meta-Analyses Of The Fusion Rates. What is the optimal graft material?

Marjan Alimi, MD; Paul Christos, PHD; Yu Moriguchi, MD, PhD

\section{Weill Cornell Brain and Spine Center}

Introduction: It has previously been shown that Minimally Invasive Transforaminal Lumbar Interbody Fusion (MIS TLIF) has similar fusion rates and complication rates compared to open TLIF. No previous studies have compared the fusion rates of different graft materials used in MIS TLIF. The aim of the current study was comparison of the fusion rate between graft materials, via meta-analysis of the published literature.

Methods: A Medline search was performed to identify the studies reporting fusion rates of MIS TLIF using different graft materials. A database was created including patient demographics, per-operative data, type of graft material, clinical outcome, fusion rate, fusion assessment modality, and duration of follow-up. Groups were defined based on the graft utilized. Meta-analysis of the fusion rate was performed.

Results: Forty series with 1320 patients were included. Age and sex distribution were homogeneous in almost all series. Significant clinical improvement was observed in all studies. Fusion rates were high, regardless of the type of the graft, ranging from $92 \%$ to $99 \%$. Comparison of all rhBMP series with all non-rhBMP series showed fusion rates of $96.6 \%$ and $92.5 \%$, respectively. Iliac bone graft alone compared to non- Iliac bone graft showed fusion rates of $92.9 \%$ and $92.4 \%$, respectively. The lowest fusion rate was seen with isolated use of autologous local bone $(91.8 \%)$. Yet, addition of rhBMP to local bone increased the fusion rate up to $95.3 \%$. The overall fusion rate was found to be $94.4 \%$ for studies using X-Rays to assess the fusion, while it was $90.8 \%$ for studies using CT-Scans. The median complication rate was $8.82 \%$.

Conclusion: Utilization of rhBMP for MIS TLIF resulted in high fusion rates. Isolated use of local bone resulted in the lowest. The modality by which the fusion rate is being evaluated, needs to be taken into consideration. Given the potential complications of iliac bone harvesting and rhBMP, the decision on the type of graft martial should be made for each patient separately. When rhBMP used, efforts should be made to use the lowest possible amount.

Poster \# 5. In vitro Biomechanical Range of Motion and Coronal Plane Cobb Angle Correction of Fusionless Anterior Tether Constructs for Controlled Scoliosis Correction

William F. Lavelle, MD; Mark Moldavsky, M.S.; Yiwei Cai, B.S.; Sean Jenkins, B.S Biomed; Nathaniel Ordway, MS; Brandon Bucklen, PhD

\section{Upstate Bone and Joint Center}

Introduction: Fusionless scoliosis correction has recently gained popularity for treating skeletally immature patients who failed bracing. Anterior tethering allows for correction while still promoting growth and mobility. Further correction may be theoretically achieved as the patient grows, in accordance with the HueterVolkmann law. The current study evaluates the in vitro range of motion (ROM) of thoracic anterior tether constructs with different tensioning techniques and changes in Cobb angles in a biomechanical model.

Methods: Eight T3-L1 cadaveric specimen (2M 6F; 57 \pm 20 years) underwent ROM testing, with an applied lateral bending moment of $6 \mathrm{Nm}$ at $1 \% \mathrm{sec}$, using a 6 degree of freedom machine. Bone screws of $5.5 \mathrm{~mm}$ diameter were implanted with bicortical purchase. The tether was tensioned to $100 \mathrm{~N}$ for and a $4.5 \mathrm{~mm}$ diameter rod was used for the rigid constructs. All specimens were tested intact and with the following constructs 1) T4-T12 rigid (T4-T12 R); 2) T4-T12 sequentially tensioned (T4-T12 SEQ T); 3) T4-T12 tensioned at T12, (T4-T12 T); and 4) T4-T12 no tension (T4-T12 No T). Repeated measures ANOVA with Tukey's post hoc analysis was used to determine significant differences in ROM, a student $\mathrm{t}$-test assuming equal variances was used for comparing Cobb angles $(\mathrm{p} \leq 0.05)$.

Results: During lateral bending, the normalized ROM for intact was $100( \pm 33.30) \%$. The T4-T12 R construct reduced motion to $28.47( \pm 8.87) \%$. The T4-T12 SEQ T, T4-T12 T, and T4-T12 No $\mathrm{T}$ constructs resulted in a stepwise increase in motion to $44.18( \pm 15.34) \%, 47.33( \pm 17.62) \%$, and $70.94( \pm 18.85) \%$, respectively, compared to intact. All constructs significantly reduced motion compared to intact $(\mathrm{p} \leq 0.05)$. The T4-T12 No T motion construct was significantly more compared to the other three instrumented constructs $(\mathrm{p} \leq 0.05)$. The average change in T4-T12 Cobb angle from intact was $4.6( \pm 3.2)^{\circ}$ and $9.9( \pm 5.5)^{\circ}$ for the T4-T12 $\mathrm{T}$ and T4-T12 SEQ T constructs respectively $(\mathrm{p}=0.05)$.

Conclusion: Anterior tethering significantly reduces motion compared to intact but allows for motion greater than rigid rod constructs. Significantly more correction was achieved with sequential tensioning and the majority of the change occurred at the superior levels. When performing surgery with anterior tether, it may be time consuming to tension at every level without a significant biomechanical advantage. Radiographic evaluation of coronal plane correction and clinical studies are needed to fully understand the immediate and long-term effect of tethered constructs.

Normalized Lateral Bending ROM 120

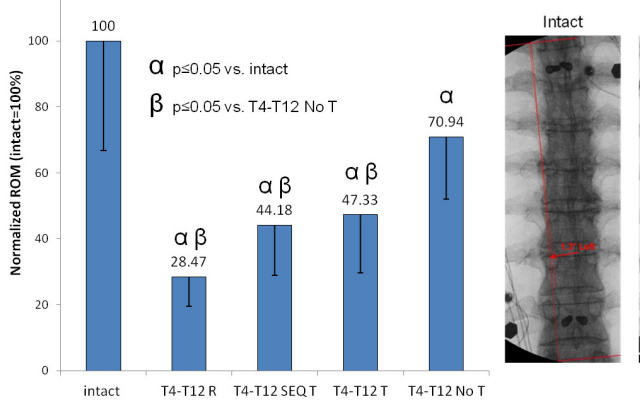

\section{Coronal Plane Cobb Angles}


Poster \# 6. Minimally Invasive versus Open Single-Level Transforaminal Lumbar Interbody Fusion: Surgical Outcomes, Narcotic Utilization, and Functional Capacity

Junyoung Ahn, BS; Andrew Joon Park, BS; Vincent Rossi, BS, BA; Ehsan Tabaraee, MD; Gabriel Duhancioglu, MS; Rahul Kamath, MS; Aamir Iqbal, BS; Blaine T. Manning, BS; Spencer Leblang, $B S$; Kern Singh, $M D$

\section{Rush University Medical Center}

Introduction: Evidence suggests that minimally invasive techniques are associated with minimal soft tissue disruption and expeditious post-operative recovery period. However, few studies have compared the functional outcomes, as evaluated by an independent physical therapist, and narcotic utilization between open versus minimally invasive transforaminal lumbar interbody fusion (MISTLIF). The purpose of this study is to compare the surgical and functional outcomes following a primary 1-level transformational lumbar interbody fusion between open and minimally invasive techniques.

Methods: A retrospective cohort analysis of 406 consecutive patients who underwent a primary single level MIS-TLIF for degenerative etiologies between 2008-2014 was performed utilizing a prospectively maintained registry. Patients were stratified into cohorts based upon surgical approach (open or MIS) and assessed with regards to demographics comorbidity, surgical outcomes, post-operative narcotics utilization, and functional capacity evaluation criteria as defined by the U.S. Department of Labor. Statistical analysis was performed with independent sample t-tests for continuous variables and chi-square analysis for categorical data. A p-value $<0.05$ denoted statistical significance.

Results: Of the 406 patients, $48(11.8 \%)$ and $358(88.2 \%)$ underwent open and MIS TLIF, respectively. The open cohort was significantly younger and demonstrated a lower comorbidity burden when compared to the MIS cohort $(\mathrm{p}<0.05)$. The MIS cohort was associated with a reduced procedural time (119.8 \pm 47.1 vs. $139.1 \pm 29.9 \mathrm{~min}, \mathrm{p}<0.05)$ and estimated blood loss $(73.7 \pm 84.2$ vs. $288.5 \pm 186.5 \mathrm{cc}, \mathrm{p}<0.05)$. Peri-operative narcotics utilization and

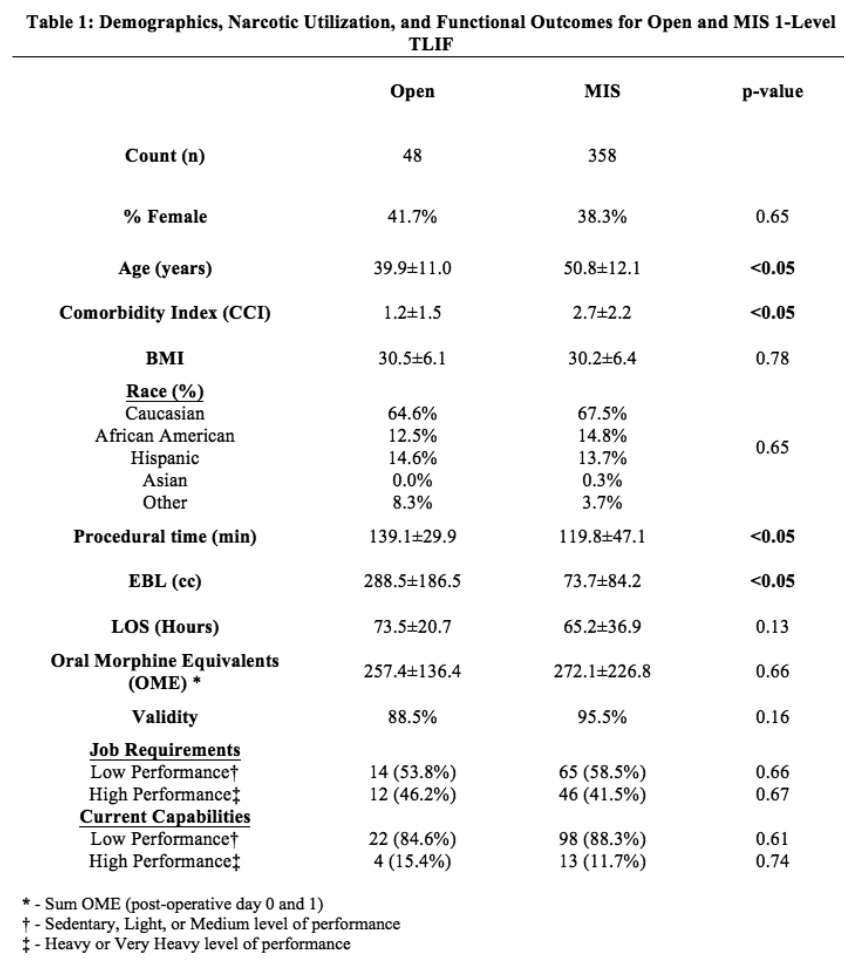

length of hospitalization was comparable between cohorts. The functional outcomes did not vary as a function of surgical approach.

Discussion: Following a 1-level TLIF, open and MIS approaches demonstrated similar final work capabilities despite the open cohort experiencing greater operative time and blood loss. Additionally, narcotics utilization in the immediate post-operative period and the length of hospitalization was comparable between the surgical cohorts.

Conclusion: Further studies are warranted to characterize the factors that are predictive of meeting functional job requirements following a 1-level TLIF.

Poster \# 7. The Duel-energy X-ray Absorptiometry (DEXA) T-score as a Predictor of Graft Subsidence Following Minimally Invasive Lateral Lumbar Interbody Fusion (LLIF)

Zachary J. Tempel, MD; Gurpreet S. Gandhoke, MD; Bryan D. Bolinger, DO; David O. Okonkwo, MD PhD; Adam S. Kanter, MD

\section{University of Pittsburgh Medical Center}

Introduction: The LLIF procedure is a useful stand-alone option for many spinal conditions. Recently, LLIF has been incorporated as an adjunct to posterior approaches for spinal deformity and scoliosis. One complication of LLIF is subsidence of the interbody graft into the vertebral bodies, resulting in severe pain, impaired arthrodesis and potentially fracture of the body. Low bone density, as measured by T-score on DEXA scanning, has also been postulated to increase the risk of subsidence.

Methods: A retrospective review of prospectively collected data was performed on all patients who underwent LLIF at this institution over between July 2008 and June 2014, consisting of 712 levels in 335 patients. Patients with subsidence following LLIF were recorded. We utilized the T-score obtained from the femoral neck DEXA scans, which is used to determine overall fracture risk. The T-score of patients with subsidence was compared to those without subsidence.

Results: 20 of 57 (35\%) patients without subsidence had a DEXA T-score between -1.0 and -2.4 consistent osteopenia, one patient $(1.8 \%)$ exhibited a T-score less than -2.5 , consistent with osteoporosis. 13 patients of $23(57 \%)$ with subsidence exhibited a T-score between -1.0 and -2.4 , consistent with osteopenia, five (22\%) exhibited a T-score of -2.5 or less, consistent with osteoporosis. The mean DEXA T-score in patients with subsidence was -1.65 $(\mathrm{SD}=1.04)$ compared to -0.45 ( $\mathrm{SD}=0.97)$ in patients without subsidence $(\mathrm{p}<0.01)$. The area under the Receiver Operating Characteristic curve for patients with a T-score of -1.0 or less was $80.1 \%$

Conclusions: Patients with DEXA T-scores less than -1.0 who
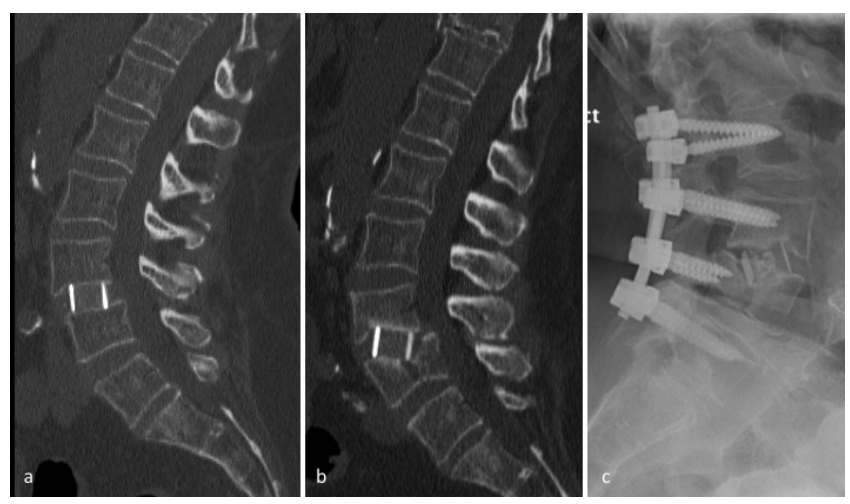

Figure 1. (a) Immediate postoperative CT scan in a 63 year-old female with osteoporosis who underwent a single-level LLIF at L3/4. (b) CT scan 3 weeks after surgery revealing graft subsidence and vertebral body fracture. (c) Postoperative radiographs following open fixation. 
undergo stand-alone LLIF are at a much higher risk of developing graft subsidence. Further, they are at an increased risk of requiring additional surgery. In patients with poor bone quality, consideration could be made to supplement the LLIF cage with posterior instrumentation.

\section{Poster \#8. Lumbar Spine Injuries From Simulated Under-body Blast Loading}

Narayan Yoganandan, PhD; Frank A. Pintar, PhD; Jason Moore, MS; Jamie L. Baisden, MD; dennis maiman, md phd; Jiangyue Zhang, PhD; Andrew C. Merkle, MS

Medical College of Wisconsin

Introduction: Clinical studies have shown increased prevalence of lumbar injuries due to underbody blast loadings. While injuries are reported and mechanisms are postulated, there is a paucity of research data confirming the hypotheses and studies are needed to improve the safety of our soldiers. The study was conducted to reproduce such injuries and determine the associated forces, moments, and injury mechanisms due to under-body blast loading.

Methods: Human cadaver osteo-ligamentous lumbar columns were obtained, and x-rays and CTs were taken. Specimens were fixed at the two ends: T12 and sacrum. Military volunteers demonstrated the normal posture of a seated soldier while wearing personal protective equipment and this information was used to align the lumbar columns into realistic postures. T12-L1 and L5-S1 joints were unconstrained. Bony targets were used to monitor temporal kinematics. Six-axis load cells at T12 and sacrum was used to obtain forces and moments. A custom vertical accelerator was used to apply inferior-to-superior loadings, simulating under-body blast events. A repeated testing protocol was adopted by applying gradually increasing accelerations at the sacrum. Intermittent $\mathrm{x}$-rays and palpation were used to check integrity. After the final test that resulted in the diagnosis of injuries, CT scans were obtained and dissection was performed.

Results: Mean age, stature, total body mass and BMI: 61 years, $1.8 \mathrm{~m}, 71 \mathrm{~kg}$ and $22 \mathrm{~kg} /$ square-meter. Injuries occurred at L1-L3 levels: burst fractures, T12-L1 joint injuries and facet fractures with ligament distraction, and disc and facet joint involvements. Axial forces and sagittal moments (sacrum: 1.5-5.6 kN and 20-390 $\mathrm{Nm}$; T12: 1.4-4.4 kN and 70-310 Nm) were predominant metrics characterizing mechanism of load transfer along column. Temporal kinematics showed that the spinal column sustained compressive displacements up to $25 \mathrm{~mm}$.

Discussion-Conclusion: This study simulated under-body blast loading using a vertical accelerator, aligned specimens simulating soldiers' spinal posture, recorded dynamic forces and bending moments at either ends of the column, determined temporal segmental motions and produced anterior and posterior column injuries similar to those seen from under-body blast events, characterizing lumbar column kinetics and improving our understanding of injuries, injury mechanisms, and spine tolerances applicable to military populations.
Poster \#9. Contrast-Enhanced MicroCT Characterization of Cartilage Endplate Morphology and Extracellular Matrix Composition

Michael D. Newton, BS; Abigail Davidson, BS; Tristan Maerz, M.S. Eng; Daniel Park, MD; Kevin Baker, Ph.D.

Beaumont Health

Introduction: Intervertebral disc degeneration is accompanied by molecular and morphological changes to the cartilage endplates, inducing the loss of sulfated glycosaminoglycan (sGAG) from the extracellular matrix. Characterization of endplate changes in preclinical models could provide a better understanding of the IVDendplate relationship, but current imaging methods are limited in spatial resolution. Quantitative contrast-enhanced micro-CT $(\mu \mathrm{CT})$ has previously demonstrated a strong correlation to sGAG content in the IVD and articular cartilage, based on the preferential exclusion of an anionic contrast agent from sGAG-rich tissues. The objective of this study was to characterize sGAG levels and morphology of the rat cartilage endplate using $3 \mathrm{D}$ contrast-enhanced $\mu \mathrm{CT}$.

Methods: The contrast agent used in this study was ioxaglate, diluted to $40 \% \mathrm{v} / \mathrm{v}$ in $0.01 \mathrm{M}$ phosphate buffered saline. Lumbar IVDs and their endplates were dissected under microscopy from adult, female Lewis rats. One set of endplates was digested for 24 hours in papain to deplete sGAG content within the endplate thus simulating the degenerative state, while another set was analyzed undigested as a healthy control. Endplates were scanned via $\mu \mathrm{CT}$ before and after 24-hour contrast incubation in ioxaglate. Pre- and post-contrast enhancement image stacks were co-registered using MATLAB. 3D endplate volumes were isolated via manual outlining and grayscale thresholding. For each volume, mean attenuation (increase over baseline) was calculated, and thickness analysis was performed via BoneJ.

Results: Ioxaglate enables marked contrast enhancement of the vertebral endplate as our group has previously shown. Papain digestion produced a $66 \%$ increase in attenuation compared to healthy controls (Fig 1A). After contrast-enhancement, the increase in attenuation over baseline was $2997 \pm 239 \mathrm{HU}$ for control endplates and $4994 \pm 324 \mathrm{HU}$ for digested endplates $(\mathrm{P}=0.025)$. $\mu \mathrm{CT}$ imaging also enabled effective morphological characterization of the endplate (Fig 2A).

Conclusions: Contrast-enhanced $\mu \mathrm{CT}$ enables effective segmentation of the endplate and provides high resolution characterization of endplate morphology sGAG content. The use of contrastenhanced $\mu \mathrm{CT}$ to characterize endplates in preclinical models could provide valuable information about the nature of degenerative changes in the vertebral endplate and their role in IVD degeneration. The high-resolution nature of this imaging facilitates its use in small animals frequently employed early in preclinical testing.
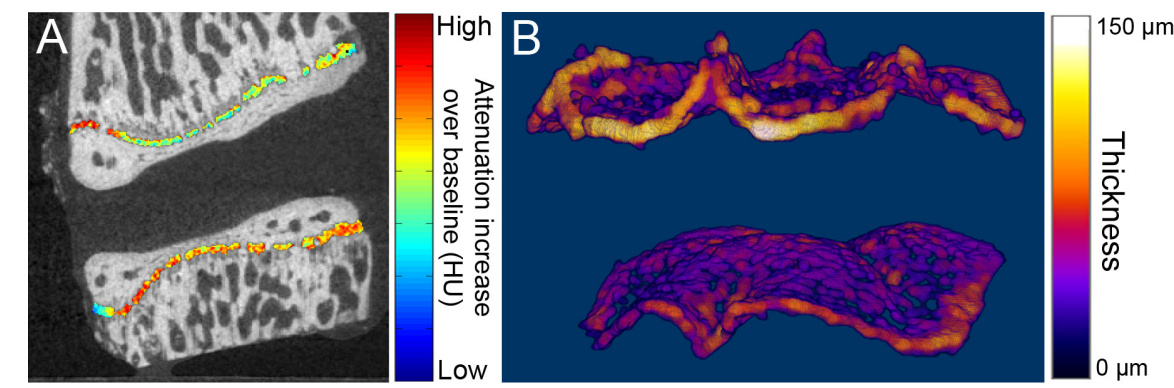

Figure 1. Contrast-enhanced $\mu C T(A)$ and 3D thickness maps $(B)$ of cranial (above) and caudal (below) cartilage endplates. Contrast enhanced $\mu$ CT provides a quantitative metric of sulfated glycosaminoglycan content in the endplates and facilitates 3D morphological assessment. 
Poster \#10. A Prospective Multi-center Clinical and Radiographic Outcomes Evaluation of chronOS ${ }^{\mathrm{TM}}$ Strip for Lumbar Spine Fusion

Adam S. Kanter, MD; Gurpreet S. Gandhoke, MD; William C. Welch, M.D.; Paul M. Arnold, M.D.; Joseph S. Cheng, M.D., M.S.; David O. Okonkwo, MD PhD

University of Kansas Medical Center

Introduction: The use of beta-tricalcium phosphate $(\beta-\mathrm{TCP})$ in single-level posterolateral instrumented fusion has shown fusion rates and clinical outcomes comparable to autologous iliac crest bone. chronOS ${ }^{\mathrm{TM}}$ Strip is a synthetic bone void filler made from $\beta$-TCP granules and a resorbable polymer, resulting in a porous, osteoconductive, flexible 3-dimensional composite with excellent memory characteristics.

Methods: This prospective, multi-center clinical outcomes study evaluated fusion rates following the use of chronOS ${ }^{\mathrm{TM}}$ Strip in patients with degenerative disc disease who underwent instrumented posterolateral spinal fusion with interbody support up to two consecutive levels between L2-S1. The chronOS ${ }^{\mathrm{TM}}$ Strip, combined with bone marrow aspirate and local bone, was applied to the posterolateral gutters. Patients were evaluated preoperatively and at 6 weeks, 6, 12, and 24 months postoperatively. An independent radiologist assessed posterolateral and intervertebral fusion using dynamic x-rays supplemented with CT imaging. Posterolateral fusion success included evidence of posterolateral bridging bone, dynamic radiographs, and posterior hardware condition. Standardized outcome measures assessed pain, function, and neurological status at all visits.

Results: Seventy-six (76) patients were enrolled and treated at 13 sites. At 24 months, 55/76 patients (72\%) were evaluated; 49/76 $(65 \%)$ had sufficient data to determine the primary endpoint. There were no adverse events related to the chronOS ${ }^{\mathrm{TM}}$ Strip. Three patients underwent secondary surgery at the index level for infection (1), interbody cage migration (1), or nerve root impingement (1). There were no deaths. Posterolateral fusion success was achieved in 48/54 $(88.9 \%)$ patients at 12 months and in $45 / 49$ (91.8\%) patients at 24 months. Successful fusion around the interbody spacer was observed in 33/61 (54.1\%) patients at 12 months and in 37/52 (71.2\%) patients at 24 months. At all follow up time points, statistically significant improvements were observed in back/leg pain and functional status as measured by VAS, ODI and SF-12 health surveys.

Conclusions: Composite bone void filler, when applied posterolaterally with bone marrow aspirate and local bone and concomitant interbody support, can be used to achieve successful posterolateral fusion and improved clinical outcomes in patients with degenerative disc disease.

Poster \# 11. Occupant and Crash Characteristics of Elderly Subjects with Thoracic and Lumbar Spine Injuries Following Motor Vehicle Collisions

Raj Rao, MD; Evan H. Sobel, BA; Chirag A. Berry, MD; Narayan Yoganandan, $\mathrm{PhD}$

\section{Medical College of Wisconsin}

Background Context: By the year 2030 it is anticipated that $20-25 \%$ of all licensed drivers will be over the age of 65 . Despite driving less than the young, older drivers are involved in a higher proportion of crashes than young drivers. Notwithstanding the safety features in modern vehicles, $15.8-51 \%$ of all thoracic and lumbar (T and L) spine injuries result from motor vehicle collisions (MVC).

Purpose: To investigate the incidence and pattern of $\mathrm{T}$ and $\mathrm{L}$ spine injuries among elderly subjects involved in MVC.

Study Design/Setting: Retrospective study of a prospectively gathered database.

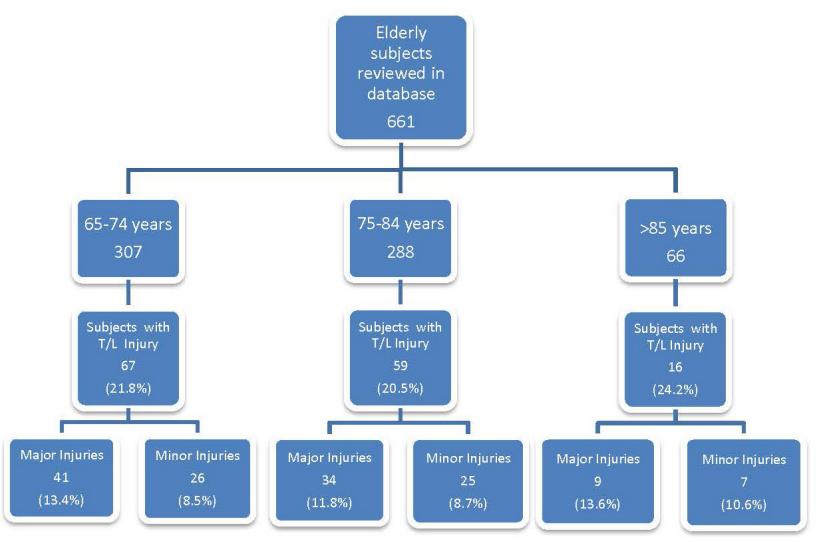

Figure 1: Schematic distribution of 661 subjects with thoracic and lumbar spine injuries reviewed in CIREN database.

Patient Sample: One hundred and forty-two occupants 65 years and older with T and L spine injuries from 4,572 occupants in the Crash Injury Research and Engineering Network (CIREN) database between 1996 and 2011.

Outcome Measures: No clinical outcome measures were evaluated in this study.

Methods: The CIREN database is a prospectively maintained, multi-centered database that enrolls occupants of MVC who have sustained moderate to severe injuries. The database was queried for $\mathrm{T}$ and $\mathrm{L}$ spine injuries in subjects 65 and older. The CIREN files for all elderly individuals were reviewed for demographic, injury and crash data. Each occupant's T and L injury was categorized using a modified Denis classification.

Results: Of 661 elderly subjects in the database, $142(21.48 \%)$ sustained $\mathrm{T}$ and $\mathrm{L}$ spine injuries. Of the 102 major injuries there were 63 compression, 20 burst and 12 extension fractures. Seatbelt use predisposed elderly subjects to compression and burst fractures while seatbelt and airbag use together predisposed to burst fractures. The deployment of airbags without concurrent seatbelt use appeared to predispose elderly subjects to neurologic injury, higher ISS and higher mortality. Occupants using three-point belts who had airbags deploy during the collision had the lowest rates of fatality and neurologic injury.

Conclusions: $\mathrm{T}$ and $\mathrm{L}$ spine injuries in the elderly are not uncommon despite restraint use. While seatbelts used alone and in conjunction with airbag deployment reduced fatalities and neurologic injuries in the elderly, they were associated with compression and burst fractures in the $\mathrm{T}$ and $\mathrm{L}$ spine.

Poster \# 12. Unilateral versus Bilateral Pedicle Screw Instrumentation for a Minimally Invasive Transforaminal Lumbar Interbody Fusion: Analysis of Surgical Outcomes, Costs, and Narcotic Utilization

Junyoung Ahn, BS; Andrew Joon Park, BS; Vincent Rossi, BS, BA; Ehsan Tabaraee, MD; Sreeharsha V. Nandyala, BA; Daniel D. Bohl, MPH; Khaled Aboushaala, MD; Kern Singh, MD

\section{Rush University Medical Center}

Introduction: The efficacy of a minimally invasive transforaminal lumbar interbody fusion (MIS-TLIF) for the management of degenerative lumbar stenosis has been described. However, the differences in peri-operative outcomes, costs, and narcotic utilization between unilateral and bilateral instrumentation in the setting of a 1-level MISTLIF have not been well characterized. The purpose of this study is to compare the surgical outcomes, narcotic utilization, and costs between a unilateral and bilateral instrumented 1-level MIS-TLIF. 
Table 1 - Demographics, Surgical Outcomes, Narcotic Utilization, and Total Costs

\begin{tabular}{|c|c|c|c|}
\hline & Unilateral & Bilateral & P-value \\
\hline Count (n) & 183 & 40 & \\
\hline Age (years) & $50.7 \pm 12.9$ & $49.3 \pm 10.1$ & 0.50 \\
\hline$\%$ Female & $43.7 \%$ & $42.5 \%$ & 0.89 \\
\hline$\%$ Caucasian & $71.8 \%$ & $63.2 \%$ & 0.67 \\
\hline Comorbidity Index (CCI) $\dagger$ & $2.9 \pm 2.4$ & $2.8 \pm 2.0$ & 0.92 \\
\hline Smoker (\%) & $28.9 \%$ & $30.0 \%$ & 0.90 \\
\hline Pre-operative VAS & $6.9 \pm 1.9$ & $7.2 \pm 2.1$ & 0.36 \\
\hline Procedural Time (min) & $106.9 \pm 47.5$ & $145.7 \pm 45.1$ & $<0.001$ \\
\hline Estimated Blood Loss (cc) & $59.7 \pm 58.3$ & $152.7 \pm 176.0$ & $<0.001$ \\
\hline Length of Hospitalization (hours) & $66.8 \pm 38.1$ & $75.6 \pm 60.9$ & 0.24 \\
\hline $\begin{array}{c}\text { Oral Morphine Equivalents } \\
\text { (OME) } \ddagger\end{array}$ & $390.9 \pm 239.1$ & $426.8 \pm 271.4$ & 0.40 \\
\hline \multicolumn{4}{|l|}{ Operative Levels } \\
\hline L2-3 & $1.1 \%(\mathrm{n}=2)$ & $5.0 \%(n=2)$ & 0.09 \\
\hline L3-4 & $7.1 \%(\mathrm{n}=13)$ & $2.5 \%(\mathrm{n}=1)$ & 0.28 \\
\hline L4-5 & $48.1 \%(n=88)$ & $55.0 \%(\mathrm{n}=22)$ & 0.43 \\
\hline L5-6 & $0.5 \%(\mathrm{n}=1)$ & - & 0.64 \\
\hline L5-S1 & $43.2 \%(n=79)$ & $37.5 \%(n=15)$ & 0.51 \\
\hline \multicolumn{4}{|l|}{ Post-operative VAS } \\
\hline 6-weeks & $3.9 \pm 2.1$ & $4.0 \pm 2.3$ & 0.87 \\
\hline 12-weeks & $3.7 \pm 2.4$ & $4.2 \pm 2.7$ & 0.29 \\
\hline 6-months & $3.7 \pm 3.7$ & $3.8 \pm 2.9$ & 0.79 \\
\hline \multicolumn{4}{|l|}{ Complications } \\
\hline Intra-operative & $4.9 \%(n=9)$ & $5.0 \%(\mathrm{n}=2)$ & 0.98 \\
\hline In-hospital & $2.2 \%(n=4)$ & $2.5 \%(\mathrm{n}=1)$ & 0.90 \\
\hline 6-week & $0.5 \%(\mathrm{n}=1)$ & $5.0 \%(n=2)$ & $<0.05$ \\
\hline 12-week & 0 & 0 & - \\
\hline Neuroforaminal Bone Growth $\uparrow \dagger$ & $10.4 \%(\mathrm{n}=19)$ & $5.0 \%(\mathrm{n}=2)$ & 0.29 \\
\hline 1-year arthrodesis $\% ~ \dagger \dagger$ & $95.6 \%(n=175)$ & $92.5 \%(n=37)$ & 0.41 \\
\hline Total Direct Costs & $\$ 11,641 \pm 8,573$ & $\$ 18,888 \pm 15,474$ & $<0.05$ \\
\hline
\end{tabular}

Methods: 223 consecutive patients who underwent a primary 1-level MIS-TLIF for degenerative etiologies between 2007-2012 were identified utilizing a prospectively maintained clinical registry. Patients were stratified into unilateral and bilateral pedicle screw instrumentation cohorts. Demographics, smoking status, comorbidity, surgical outcomes, narcotic utilization, complications, costs, and arthrodesis rates were assessed between cohorts. Statistical analysis was performed with Student's T-test for continuous variables and Pearson's Chi-square analysis for categorical data. An alpha level of $\mathrm{p}<0.05$ denoted statistical significance.

Results: Of the 223 patients, $183(82.1 \%)$ and $40(17.9 \%)$ underwent unilateral and bilateral instrumentation, respectively. Demographics, comorbidity, smoking status, operative levels, VAS scores, and narcotic utilization were similar between cohorts. The unilateral patients demonstrated a shorter operative time (106.9 \pm 47.5 vs. $145.7 \pm 45.1 \mathrm{~min}, \mathrm{p}<0.001)$, lower estimated blood loss (EBL) $(59.7 \pm 58.3$ vs. $152.7 \pm 176.0 \mathrm{cc}, \mathrm{p}<0.001)$, and lower 6 -week complication rates than the bilateral cohort. The 1-year arthrodesis rates were similar between cohorts. However, bilateral instrumentation was associated with greater costs.

Discussion: Unilateral patients demonstrated decreased hospital resource utilization and comparable surgical outcomes when compared to bilateral patients. The bilateral cohort incurred increased costs likely attributed to additional implants, operating and anesthesia time, radiology, and surgical services. However, narcotic utilization appears to be independent of instrumentation.

Conclusions: Findings of this analysis suggest that unilateral instrumentation may be associated with similar clinical outcomes as compared to bilateral instrumentation in the setting of stable, lowgrade spondylolisthesis and degenerative disc disease.
Poster \# 13. Comparing Various Surgical Management Options for Lumbar Degenerative Spondylolisthesis

John R. Burleson, MD; Peter J. Wagner, MD; Patrick Connolly, MD; Christian Dipaola, MD; Michael Stauff, MD

UMass Medical Center

Introduction: There are multiple options for surgical treatment in patients with lumbar degenerative spondylolisthesis. The options include: decompression, decompression with a non-instrumented fusion, and decompression with an instrumented fusion. Often surgeons also perform interbody fusions from various approaches, including: anterior, antero-lateral, lateral, transforaminal, and posterior. The decision regarding the optimal surgical treatment is based on multiple variables, but differs between surgeons. The purpose of this study is to compare the outcomes of patients with lumbar degenerative spondylolisthesis who are treated with different surgical techniques.

Methods: We performed an ambispective review of the UMass Spine Center registry for patients with degenerative spondylolisthesis who were treated surgically at our institution from 2006-2013. The patients were stratified based on procedure. We compared the cohorts using the following variables: demographics, rate of reoperation, levels of fusion, and pre/post operative SF-36 score.

Results: Our review captured 154 patients (74\% female). The average age for all patients was 61years old. The surgical procedures were performed by five fellowship trained orthopedic spine surgeons. Follow-up ranged from 1-6 years with an average of 2.4 years. Patients in every group saw an improvement in their SF-36 scores at the latest follow up.

Discussion \& Conclusion: Our results demonstrate that all patients had significant improvement from pre-operatively to 1 year post-operatively except for the cohort that had an ALIF with posterior instrumented fusion. The re-operation rate was higher with increasingly complex surgical procedures. Further prospective research is needed in order to determine the optimal surgical treatment for each patient with lumbar degenerative spondylolisthesis who fails non-operative treatment.

\begin{tabular}{|l|c|c|c|c|c|c|c|}
\hline \multicolumn{1}{|c}{ Procedure } & Number & \multicolumn{1}{c}{ Avg age } & Pre-op SF-36 & 1 Year SF-36 & P value Re-op Rate \\
\hline Decompression w/ non-instrumented Fusion & 55 & 68 & 29.12 & 38.26 & $<0.01$ & $9.10 \%$ \\
\hline Decompression with posterior instrumented fusion & 48 & 61 & 27.98 & 35.66 & $<0.01$ & $14.60 \%$ \\
\hline Decompression with T/PLIF & 35 & 49 & 29.85 & 36.27 & $<0.01$ & $20 \%$ \\
\hline ALIF & 10 & 53 & 24 & 33.91 & 0.03 & $20 \%$ \\
\hline ALIF with posterior instrumented fusion & 6 & 49 & 25.76 & 28.5 & 0.58 & $50 \%$
\end{tabular}

Poster \# 14. Intraoperative Cone Beam CT And Image-guided Surgery: How Accurate Is It For Virtual Versus Actual Pedicle Screw Placement?

Catherine Miller, MD; Matthew Allan Hunt, MD; Charles Ledonio, MD; David Polly, MD

University of Minnesota

Background: Technological advances, including navigation, have been made to improve safety and accuracy of pedicle screw fixation. We evaluated the accuracy of the virtual screw placement (Stealth projection) compared to actual screw placement (intra-operative $\mathrm{O}$-Arm) and examined for differences based on the distance from the reference frame.

Methods: A retrospective evaluation of prospectively collected data was conducted from January 2013 to September 2013. We evaluated thoracic and lumbosacral pedicle screws placed using intraoperative $\mathrm{O}$-arm and Stealth navigation by obtaining virtual screw projections and intraoperative $\mathrm{O}$-arm images after screw placement. The screw trajectory angle to the midsagittal line and 
superior endplate was compared in the axial and sagittal views, respectively. Percent error and Intraclass correlation coefficient reliability testing (ICC) were then performed.

Results: Thirty-one patients with 240 pedicle screws (146 thoracic, 94 lumbosacral) were analyzed. The mean angular difference between the virtual and actual image in all screws was $2.17^{\circ}( \pm 2.2)$ on axial images and $2.16^{\circ}( \pm 2.2)$ on sagittal images. There was excellent agreement between actual and virtual pedicle screw trajectories in the axial and sagittal plane with ICC $=0.99$ (95\% CI: 0.992$0.995)(\mathrm{p}<0.001)$ and ICC $=0.81(95 \%$ CI: 0.759-0.855) $(\mathrm{p}<0.001)$ respectively. The axial and sagittal angular differences (\%error) were the same at $1.1 \%$ and $1.2 \%$, respectively. When comparing thoracic and lumbar screws, there was a significant difference in the sagittal angulation between the two distributions, however there were no pedicle screw breaches (anterior, lateral, medial or superior). No statistical differences were found in relation to the distance from the reference frame.

Conclusions: The virtual projection view is clinically accurate compared to the actual placement on intra-operative CT in both the axial and sagittal views. There is slight imprecision $\left(2.17^{\circ}\right)$ in the axial plane and a minor difference in the sagittal thoracic and lumbar angulation, although all screws were still in optimal position within the pedicle with no breaches. Distance from the reference frame did not affect the accuracy of the screw placement.

Poster \# 15. Accuracy of Percutaneous Pedicle Screw Placement: Does Training Level Matter?

Evan Baird, MD; Steven McAnany, MD; Samuel Overley, MD; Javier Guzman, B.S.; Sheeraz Qureshi, MD, MBA

\section{Mount Sinai Medical System}

Background Context: Current literature varies widely in the reported frequency of facet violation during placement of percutaneous pedicle screws. However, as of yet there are no studies examining the effect that training level has on accuracy of placement.

Purpose: To assess the accuracy of percutaneous pedicle screw placement in a human cadaveric model using standard fluoroscopic guidance technique, compared across varying levels of experience.

Study Design: In vitro human cadaveric surgical technique study

Methods: Four surgeons with differing levels of training (PGY2, PGY-4, fellow, attending) were evaluated on their accuracy of percutaneous placement of screws in a uniform manner. Each of 10 cadavers was instrumented from L1-S1 bilaterally, for a total of 120 screws. Specimens were dissected to evaluate for facet and pedicle wall violations. These were then recorded and analyzed to evaluate for correlation amongst participating surgeons, laterality, spinal level, and cadaver body mass index (BMI). The authors did not receive grants or outside funding in support of their research for or preparation of this manuscript, nor do the authors have any other conflicts related to this manuscript.

Results: Of 120 screws placed, there were 35 total violations (26 superior articular facet violations $(21.7 \%), 5$ intra-articular facet joint violations (4.2\%), and 4 pedicle breaches (3.3\%)). Among the trainees there was no difference in the likelihood of causing a violation $(p=0.8863)$ but there was a difference when compared with the attending surgeon $(\mathrm{p}=0.0175)$. Laterality $(\mathrm{p}=0.1598)$, spinal level $(\mathrm{p}=0.3536)$, and BMI $(\mathrm{p}=0.8547)$ did not correlate with the likelihood of a violation.

Conclusions: Surgeons of differing training levels are able to safely and accurately place lumbar pedicle screws in a percutaneous manner, with a low likelihood of facet and pedicle wall violations. We feel that this is a safe and worthwhile skill to teach orthopaedics residents, as a supplement to learning to place pedicle screws in the traditional open manner.
Poster \# 16. Predictors of Length of Hospital Stay and Return to OR in Adult Spinal Deformity Surgical Patients: An Analysis of Patients Using the American College of Surgeons Database

Nancy J. Worley, MS; Cyrus Jalai, B.A.; Shaleen Vira, MD; Peter G. Passias, $M D$

\section{NYU Hospital for Joint Diseases}

Introduction: ASD surgery is associated with a high rate of complications which may require reoperation. Increased scrutiny is being placed on readmission, reoperation, and length of hospital stay (LOS). Efforts to optimize surgical outcomes with respect to these variables are highly warranted. This study was a retrospective review of adult spinal deformity (ASD) patients in the American College of Surgeons (ACS) database. This study identified risk factors for increased LOS and reoperation for ASD patients who underwent corrective surgery.

Methods: The ACS database was queried for diagnoses specific for adult scoliosis. LOS, demographic information, operative time, and preoperative variables including co-morbidities were collected for patients with normal and extended LOS ( $\geq 8$ days). A sub-analysis was performed for patients who were re-admitted to identify potential risk factors. Finally, among the re-admitted patients, sub-stratification was done based on patients who underwent reoperation, and significant predictors of reoperation were identified. Univariate and multivariate regression analysis was used to identify predictors.

Results: 963 patients with ASD were identified (mean age 57.3, BMI 27.5, 69.7\% female). The range of LOS was 0-64 days. The overall LOS for all patients was 6.95 days (4.11 for patients with normal LOS, 12.93 for patients with extended LOS). Multivariate linear regression identified prior operation within 30 days $(\mathrm{P}<0.001)$ and operative time $(\mathrm{P}<0.001)$ as significant predictors of extended $\operatorname{LOS}$ ( $\geq 8$ days). Odds ratios for these predictors are as follows: prior operation within 30 days: 7.840 [16.352-3.759], and operative time: 1.005[1.007-1.003]. 301 patients had 30-day readmission data of

\begin{tabular}{|c|c|c|c|c|}
\hline & ALL & $\begin{array}{c}\text { Normal } \\
\text { LOS }(<8 \\
\text { d) }\end{array}$ & Extended LOS $\mathbb{8}^{8}$ d) & $\begin{array}{l}\text { P- } \\
\text { value }\end{array}$ \\
\hline Overall (\%) & $963(100.09 \%)$ & $\begin{array}{c}653 \\
(67.8 \%) \\
\end{array}$ & $310(32.2 \%)$ & \\
\hline Age ( $( \pm \mathrm{SD})$ & $57.38=17.22$ & $\begin{array}{c}56.46= \\
18.04 \\
\end{array}$ & $59.33=15.19$ & 0.010 \\
\hline Alcobol history (\%) & $46.4 \%$ & $49.0 \%$ & $41.0 \%$ & 0.019 \\
\hline Hypertension (\%) & $48.1 \%$ & $45.6 \%$ & $53.2 \%$ & 0.028 \\
\hline Pulmonary Comorbidities (\%) & $5.3 \%$ & $4.2 \%$ & $7.7 \%$ & 0.028 \\
\hline Prior Op within 30d (\%) & $9.9 \%$ & $3.2 \%$ & $22.1 \%$ & $<0.001$ \\
\hline ASA Clasa $\geq 3(9)$ & $51.2 \%$ & $46.2 \%$ & $61.6 \%$ & $<0.001$ \\
\hline ASA Class (meen = SD) & $2.50=0.62$ & $\begin{array}{l}2.44 \pm \\
0.626 \\
\end{array}$ & $2.63 \pm 0.592$ & $<0.001$ \\
\hline Operative time (mean = SD) & $325.40=164.07$ & $\begin{array}{c}294.594 \\
= \\
150.489 \\
\end{array}$ & $390.281=172.609$ & $<0.001$ \\
\hline \multirow[t]{2}{*}{ Operative time $\geq 426 \mathrm{~min}(\%)$} & $25.2 \%$ & $19.3 \%$ & $37.7 \%$ & $<0.001$ \\
\hline & $\begin{array}{c}\text { Change in LOS } \\
\text { (Chstandardized. B) } \\
\end{array}$ & \multicolumn{2}{|c|}{$\begin{array}{c}\text { Relative Effect on LOS } \\
\text { (Standardized } \beta \text { ) }\end{array}$} & \\
\hline Prior Op within 30d (9) & 0.439 & & 0.275 & $<0.001$ \\
\hline Operative time (meen \pm SD) & 0.001 & & 0.316 & $<0.001$ \\
\hline \multicolumn{5}{|c|}{ Return to OR Sub-Analysis of Readmitted Patients } \\
\hline & ALL & $\begin{array}{c}\text { No } \\
\text { Return to } \\
\text { OR }\end{array}$ & Return to OR & $\begin{array}{c}\text { P- } \\
\text { value }\end{array}$ \\
\hline Overall (\%) & $25(100.0 \%)$ & $\begin{array}{c}17 \\
(68.0 \%) \\
\end{array}$ & $8(32.0 \%)$ & \\
\hline Prior Op within 30d (\%) & $12.5 \%$ & $0.0 \%$ & $40.0 \%$ & 0.025 \\
\hline \multirow[t]{2}{*}{ ASA Class (mean $=$ SD) } & $2.56 \pm 0.583$ & $\begin{array}{l}2.41= \\
0.618\end{array}$ & $2.88=0.354$ & 0.027 \\
\hline & $\begin{array}{c}\text { Change in Retum } \\
\text { to OR } \\
\text { (Cinstandardized. B) }\end{array}$ & \multicolumn{2}{|c|}{$\begin{array}{l}\text { Relative Effect on Retum to OR } \\
\text { (Stand ardized } \beta \text { ) }\end{array}$} & $\begin{array}{l}\text { P- } \\
\text { value }\end{array}$ \\
\hline Prior Op within 30d (\%) & 0.667 & & 0.476 & 0.049 \\
\hline
\end{tabular}


which $25(2.6 \%)$ were re-admitted. This study did not identify any predictors of readmission. Within these readmitted patients, the patients who required reoperation had a higher incidence of a prior operation within 30 days $(\mathrm{P}<0.05)$.

Discussion: Surgical ASD patients who had a prior operation within 30 days were at risk for increased LOS and reoperation. Increased operating time also predicted increased LOS.

Conclusion: These factors should be considered in perioperative risk stratification and patient counseling efforts.

Poster \# 17. Biomechanical Comparison Between Posterior Long-segment Fixation, Short-segment Fixation And Shortsegment Fixation With Intermediate Screws For Treatment Of Thoracolumbar Burst Fracture: A Finite Element Analysis

Nutthee Wannaratsiri, MD; Worawat Limthongkul, MD; Weerasak Singhatanadgige, MD, Ms; Wicharn Yingsakmongkol, MD

Chulalongkorn University

Background: Thoracolumbar junction is the most common site of injury to the spine. Long-segment fixation has shown a better outcome comparing to other procedures. However, posterior shortsegment fixation with intermediate screws has recently been introduced in order to preserve adjacent levels motions. There remains limited data comparing the biomechanical properties among these treatments.

Methods: The finite element model of thoracolumbar spines from T11 to L3 was created based on computed tomography scan of a full column spine model. The density of L1 vertebral body was decrease $50 \%$ to simulate the burst fracture. Three finite element models, posterior long-segment fixation (LS), short-segment fixation (SS), and short-segment fixation with intermediate screws (SI) were established. The axial load $(1,000 \mathrm{~N})$ was applied on the superior surface of the T11 vertebral body. The inferior surface of the L3 vertebral body was fixed. Vonmises stress to each screws, rods and vertebral body were analyzed.

Results: Motion of the spine in the SS and SI were more than the LS. Lowest pedicle screws in every construct has shown to absorb highest stress increasing from LS to SS (120.04 MPa for LS , 135.81 $\mathrm{MPa}$ for SI and 145.29 MPa for SS). However, the Vonmises stress occurred along the connecting rods was lowest in SI when compared with others (65.09 MPa for SI, 89.51 MPa for LS and 110.51 MPa for SS). There was an increase in the stress at lowest pedicles in SI (162.5 $\mathrm{MPa}$ ) when compared with SS (154.31 MPa) and LS (89.78 MPa). At fracture level, the stress was found at vertebral body in LS and SS (2.85 and $2.75 \mathrm{MPa}$, respectively), while stress in SI occurred only at pedicles $(42.59 \mathrm{MPa})$ without stress at the vertebral body.

Conclusions: SI, while preserving more spinal motion compared with LS, showed better biomechanics than the SS. The stress occurred around the rods and vertebral body in SI was the least among three constructs, which may reduce complications such as kyphotic deformity and instrument failure. The stress occurred at the pedicles of the fracture level which was found only in short-segment fixation with intermediate screws indiated the need of an intact pedicle at the fracture level.

Poster \# 18. Effect of Postoperative Complications on Length of Stay after Lumbar Fusion Procedures

Adam M. Lukasiewicz, MSc; Daniel D. Bohl, MPH; Andre M. Samuel, BBA; Matthew L. Webb, AB; Bryce A. Basques, BS; Jonathan Newman Grauer, MD

Yale University School of Medicine

Introduction: Postoperative complications following spinal sur-

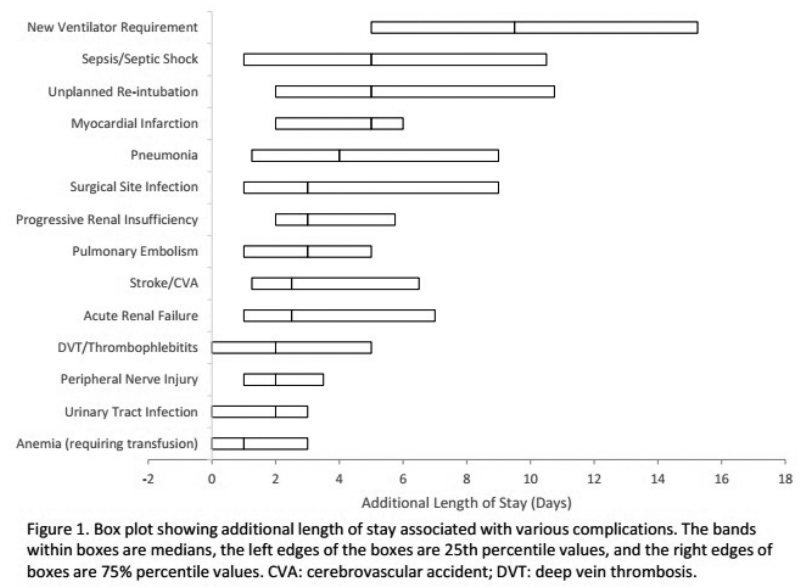

gery are widely assumed to prolong inpatient hospital stays, but the effect of various complications on length of stay in spine surgery patients have not been quantified. This study examines the association between various post-operative complications and length of stay after surgery and using a nation-wide sample.

Methods: Patient undergoing lumbar fusion procedures between the years 2010 and 2013 were identified in the American College of Surgeons National Surgical Quality Improvement Program (ACS-NSQIP) database. ACS-NSQIP is a national, prospectivelycollected database that collects data on immediate post-operative outcomes, including adverse events and length of stay. The effect of a post-operative complication was calculated as the median of the differences between the length of stay following the complication and additional length of stay in patients who were inpatient at the time of complication.

Results: In total, 14270 patients undergoing lumbar fusion procedures from 2010 to 2013 were identified in the ACS-NSQIP. The median time from surgery to discharge was 3 days.

Figure 1 shows the median additional length of stay after surgery in days for patients with a complication compared to similar patients without the complication. A new ventilator requirement resulted in the largest increase in length of day, 9.5 days. Unplanned re-intubation, sepsis, and myocardial infarction all resulted in 5 additional inpatient days, pneumonia added 4 days, pulmonary embolism, surgical site infection, and progressive renal insufficiency added 3 days each, stroke and acute renal failure each added 2.5 days, deep vein thrombosis and peripheral nerve injury added 2 days, and urinary tract infection and anemia requiring a transfusion added 1 day.

Discussion: These estimates of the additional length of stay associated with complications after lumbar fusions allow clinicians to better counsel patients and plan in the event of a complicated postoperative course. More severe adverse events, such as myocardial infarction and ventilator dependence, were generally associated with larger increases in length of stay as might be expected.

Conclusion: Clinicians should be aware of the additional length of stay expected if an adverse event occurs after lumbar fusion, and counsel patients accordingly.

Poster \# 19. Anatomical Consideration Of The Iliolumbar Venous System During Lateral Lumbar Interbody Fusion

$V u$ H. Le, MD; Jonathan Gottlieb, MD; Sohrab Pahlavan, MD; Armando Ruiz, MD; Frank Eismont, MD

University of California, Irvine

Introduction: The iliolumbar venous system is an important vascular structure that must be addressed during anterior lumbar 


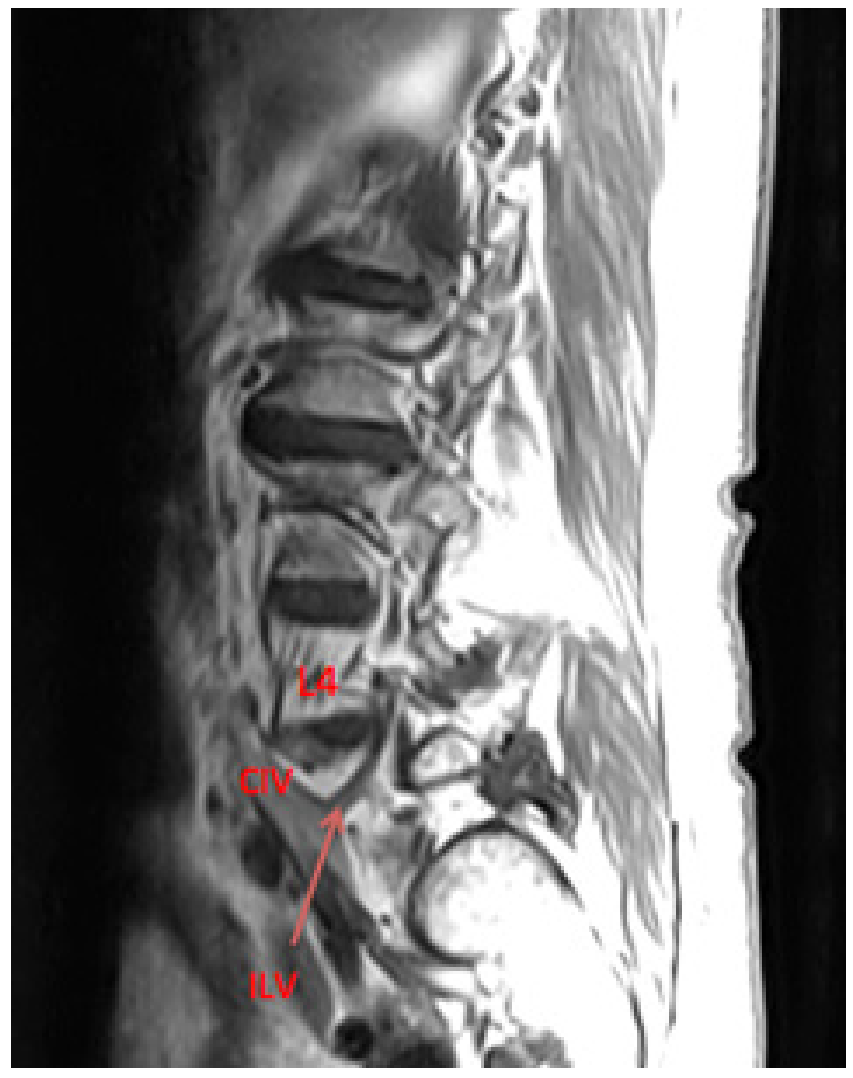

interbody fusion (ALIF), especially at the L4-L5 disc space, to prevent catastrophic bleeding. However, it is unknown whether their typical anatomical trajectories might be a hindrance during lateral lumbar interbody fusion (LLIF), especially at the anterior half of the L4-5 disc space, which is usually the working corridor of such procedures. To our knowledge, this is the first study to look at how the variants and course of the iliolumbar venous system might affect the surgical approach during LLIF.

Methods: A total of 60 random magnetic resonance imaging (MRI) studies of the lumbar spine were reviewed, consisting of 30 males and 30 females. Both left and right iliolumbar venous systems were located for each MRI, for a total of 120 iliolumbar venous systems documented. Patients with previous ALIF procedures were excluded. Parameters evaluated include terminal drainage pattern of the iliolumbar venous system, position of terminal drainage in relation to the L5 vertebral body, variants (1_single major trunk versus 2_two or more major trunks), course from the terminal drainage vein to the L4-L5 disc space, position of the tributaries at the L4-L5 disc space, and position of the tributaries at the L4-L5 disc space in relation to the psoas major muscle.

Results: All iliolumbar venous system drained into the CIV with $76.7 \%$ exhibiting variant 1 and $23.3 \%$ demonstrating variant 2 . The average position of all major trunks draining into the CIV was $14.5 \mathrm{~mm}$ (6.2) caudal to the superior end plate (SEP) of L5, with all occurring along the anterior half of the L5 vertebral body. All trunks then traversed obliquely and posterosuperiorly toward the posterior portion of the L4-5 disc space while splitting into tributaries. There, all tributaries resided in the posterior half of the disc space. All tributaries were found medial to the posterior quarter of the psoas major muscle at the L4-5 disc space. Sex and body side did not significantly affect variants and average position of drainage into the CIV ( $>0.05)$.

Conclusions: According to this study, the iliolumbar venous system was typically found away from the anterior half of the L4-5 disc space, thus, making its impact during LLIF at this level less significant than expected.
Poster \# 20. Opioid-related Adverse Events With Lumbar Spine Surgery: Is The Risk Real?

Mark F. Kurd, MD; Kevin Ong, Ph.D.; Scott Lovald, Ph.D.; Edmund Lau, MS; David Polly, MD; Kris Radcliff, MD

Rothman Institute

Introduction: Although lumbar spine surgery has positive outcomes, as many as $22.4 \%$ of unplanned readmissions within 30 days post-surgery are related to pain (Pugely 2014). The most common pain management protocol is intravenous narcotics, but this may be associated with opioid-related adverse events (ORAE), such as respiratory complications and post-operative nausea and vomiting (PONV)(Kessler 2013). This study evaluated ORAE risk using a nationally representative sample of elderly lumbar spine surgery patients.

Methods: Using the 5\% Medicare physician/carrier claims data (2010-2012), lumbar laminectomy (LL) and posterior lumbar fusion (LF) patients were identified. The 90-day postoperative risk of newly-diagnosed ORAEs was assessed, using ICD-9 diagnosis codes for respiratory complications, paralytic ileus, PONV, acute delirium, urinary retention, etc.

Results: A total of 16,765 LF and 24,514 LL Medicare patients were identified. Respiratory complications (bradypnea, pulmonary insufficiency, asphyxia, and hypoxemia) were the most commonly diagnosed ORAE (LF: $9.0 \%$ and LL: 6.3\%). Urinary retention rates of $5.8 \%$ were diagnosed for both LF and LL patients. The rate of PONV was $3.8 \%$ and $2.8 \%$ following LF and LL, respectively, while the corresponding rates of acute delirium were $3.2 \%$ and $2.1 \%$, respectively. Paralytic ileus occurred in $2.2 \%$ and $1.3 \%$ of LF and LL patients, respectively.

Conclusions: Although opioids are effective and are the most common treatment protocol for acute postoperative pain after lumbar spine surgery, the reliance on opioid analgesics exposes patients to ORAEs. These complications not only adversely affect the patient but also increase healthcare costs. Future research should explore the risk benefit ratio of alternative non-opioid pain strategies.

Poster \#21. Failure to Launch: What the Rejection of Lumbar Total Disc Replacement Tells Us About American Spine Surgery

Robert A. Hart, M.D.; John Mason DePasse, M.D.; Alan H. Daniels, M.D.

Warren Alpert Medical School of Brown University

Background: Lumbar Total Disc Replacement (L-TDR) has been available for clinical use in the United States since 2005. L-TDR has not gained wide acceptance as a surgical treatment for degenerative disc disease (DDD) despite substantial investments in product development, surgeon training, positive results in randomized controlled trials, and approval of several devices by the FDA.

Methods: Weighted estimates of the annual number of L-TDR procedures performed in the United States from 2005-2010 were calculated utilizing the NIS database. Insurance coverage policies were also assessed for L-TDR coverage via Internet search. Finally, an 18-question online survey regarding surgeons' opinions towards L-TDR was anonymously distributed to the spine surgeon members of the North American Spine Society (NASS).

Results: The estimated total number of primary L-TDR procedures performed in the US decreased from 3,650 in 2005 to 1,863 in 2010, while revision L-TDR procedures increased from 420 in 2005 and 499 in 2010. Of the 14 major insurers assessed, 11 (78.6\%) do not currently provide coverage for L-TDR. In total, 613 spine surgeons responded to the survey consisting of 439 orthopedic surgeons, 148 neurosurgeons, and 26 surgeons who received training in both fields. Over half of respondents $(51.1 \%: 313 / 612)$ have 
performed L-TDR in their career, although only $44.6 \%(136 / 305)$ of initial adopters currently perform the surgery in their practice. Despite this, $58.2 \%(169 / 289)$ of initial adopters and $81.5 \%$ $(106 / 130)$ of those currently performing L-TDR have been satisfied with the clinical results of L-TDR. When asked about their perception of L-TDR, $65.0 \%(367 / 565)$ indicated that there continues to be a lack of insurance coverage for L-TDR in their region, $54.9 \%$ (310/565) worry about the long term complications of L-TDR, and $52.7 \%(298 / 565)$ worry about the technical challenges of revising a failed prosthesis.

Conclusions: Despite enthusiasm among surgeons and engineers at the time of introduction of L-TDR in the U.S., wide adoption has not occurred. A primary reason for this failure of adoption appears to be a lack of coverage by many insurers, despite intermediate-term clinical success in FDA trials. In addition, many spine surgeons continue to express concerns regarding long-term outcomes from this procedure, as well as the technical difficulties associated with revising a failed prosthesis. This case study of a failed surgical innovation may signal increasing involvement of health care payers in clinical decision making and may be instructive to surgeons, policymakers, and medical device manufacturers.

\section{Poster \#22. Fracture Of Fusion Mass After Hardware Removal In Patients With High Sagittal Imbalance}

Cara L. Sedney, MD; Scott D. Daffner, MD; Sanford E. Emery, $M D, M B A$

\section{West Virginia University}

Introduction: As spinal fusions become more common and more complex, so do the sequelae of these procedures, some of which remain little understood. The authors report on a series of patients who underwent removal of hardware after CT-proven solid fusion, confirmed by intraoperative findings, who went on to develop a spontaneous fracture of the fusion mass, not associated with trauma. A series of such patients has not previously been described in the literature.

Methods: A retrospective review of the surgical logs of three, fellowship-trained spine surgeons yielded 7 patients with fracture of a fusion mass after hardware removal. The medical records and radiologic studies of these patients were examined in regards to patient demographics and comorbidities, initial indication for surgery, number of total surgeries, timeline of fracture occurrence, risk factors for fracture, as well as sagittal imbalance.

Results: All 7 patients underwent hardware removal in conjunction with an extension of fusion for adjacent segment disease. All had CT-proven solid fusion of their previously fused segments, which was confirmed intraoperatively. All patients had multiple previous operations for a variety of indications. Four patients were smokers. Three patients had osteoporosis. Spontaneous fracture of the fusion mass occurred without a history of trauma in all patients. These occurred 4 months to 4 years after hardware removal. All patients had significant sagittal imbalance of $13-15 \mathrm{~cm}$. The most common fracture level was L5 in six of the patients, which was the first uninstrumented level caudal to the newly placed hardware in all of these six patients. Six patients underwent surgery due to this fracture.

Conclusions: The development of a spontaneous fracture of the fusion mass may be related to sagittal imbalance and consideration should be given to re-implanting hardware for these patients, even across good fusions, to prevent spontaneous fracture of these areas if the sagittal imbalance is not corrected.

\section{Poster \# 23. Financial Implications Of Early Readmission Following Spine Surgery}

Louis G. Jenis, MD; Symeon Zannikos, MD

Newton Wellesley Hospital

Introduction: Numerous studies have been published over the last several years focusing on value in spinal surgery. Value is defined as the outcome of a specific intervention per cost over a defined period of time. One aspect of value that has been scrutinized recently is early readmission after surgery often demarcated as less than 30 days from the index procedure. As payers continue to evaluate the short and long term costs of surgical interventions, great attention has been drawn to limiting less than 30 day readmissions while agencies and insurers have considered that acute hospitalization not be reimbursed. Understanding the costs of a readmission after surgery is then critical to providing value to the patient. The purpose of this study is to identify the incidence and stratify the costs of readmission.

Methods: IRB approval was obtained. A retrospective financial / EMR database review was performed for the period of January 2012 through December 2013. Demographic and surgical data were collected for all spinal procedures performed during this time period including identification of less than 30 day readmissions etiology, diagnosis and LOS. Staged and planned readmission procedures were excluded. Readmissions were classified as surgical-related (Infection, instrumentation failure, pain) or medical. Actual direct cost included nursing time, patient supplies, OR time while indirect costs related to overhead of each department to care for these patients (electricity, housekeeping, and IS.

Results: 1,619 spine surgeries were performed. Seventy-two readmission less than 30 days were identified (11 planned and $61(3.76 \%)$ unplanned. Thirty-eight $(4.65 \%)$ occurred in 2012 and $23(2.86 \%)$ in 2013. Readmission diagnoses included pain $(n=19)$, infection $(n=19)$, instrumentation failure $(n=10)$ and medical $(n=13)$. Average readmission was $13.7+/-8.0$ days after index surgery and average readmission length of stay was 4.6+/- 3.7 days. Average actual total cost for readmission are shown in table 1.

Conclusions: The results of this study clearly document the high cost of early readmission related to spine surgery. It is critical to identify those patients that may be at risk for readmission and to develop prevention strategies. This type of financial data will be critical for all institutions to understand especially in the era of reduced hospital and physician reimbursement and negotiated episodic bundled care contracts.

$\begin{array}{ll}\text { Table 1 } & \\ \text { Diagnosis } & \text { Total costs }(\$) \\ \text { Pain } & 13,594+/-11,749 \\ \text { Infection } & 19,576+/-12,557 \\ \text { Instrumentation } & 29,581+/-36,441 \\ \text { Medical } & 25,163+/-39,215\end{array}$

Poster \# 24. Prehab As An Alternative To Elective Spine Surgery

\section{Ryan Matthew Urbonas, DO}

Swedish Neuroscience Institute

Introduction: A structured screening protocol including assessment of psychological well-being with subsequent self-directed physician supervised noninterventional treatment protocol can successfully avoid elective spine surgery while optimizing patient well-being.

Background: Certain patients with chronic LBP without focal neurologic deficits or significant instability or deformity may 
benefit from optimization of their psychological wellbeing prior to contemplating elective spine surgery. In certain cases these interventions may actually ameliorate symptoms to a degree that the originally planned surgery is no longer pursued while the patients experience a substantial improvement of their quality of life.

Material \& Methods: From Jan 2012 onwards a routine protocol at the Swedish Neurosciences Institute required new patients presenting to our Spine Clinics to complete routine psychological testing for anxiety, depression \& irritability. All patients with abnormal scores in any of the categories were placed into a structured "prehab" program with a self-directed mental \& physical health program. Only patients who had presented with degenerative lumbar disorders, such as central or foraminal spinal stenosis \& mild degenerative spondylolisthesis that corresponded with their symptoms were considered candidates for surgery.

Results: During a required minimum 1 year follow-up of 25 patients selected for a formal 'Prehab program' due to their abnormal psychological profiles we identified no cases of neurologic decline, no conversions to surgery \& confirmed statistically significant improvement in patient related outcomes scores (ODI, VAS, Opiate Index \& psychological wellness scores). Interestingly none of these patients required interventional spine management during our observation period while also increasing their functional activity profiles based on reported activity tolerances.

Conclusion: We identified 2 potential conclusions based on our early results:

1) Inclusion of wellness testing may be a valuable adjuvant to routine other screening tests for elective spine surgery.

2) Emphasis on correction of abnormal psychological profiles in a formalized 'Prehab program' may positively influence patients' subjective well-being to the point where surgical care may be delayed or not required anymore. Further investigation of a 'Mind Body Syndrome' in its spinal symptom manifestations may be warranted to optimize patient selection \& treatment outcomes.

Poster \# 25. Fixed-angle, Posteriorly-connected Anterior Cage Reconstruction Improves Stiffness And Decreases Cancellous Subsidence In A Spondylectomy Model

Matthew Colman, MD; Andrew Guss, MS; Kent Bachus, PhD; William R. Spiker, MD; Brandon D. Lawrence, MD; Darrel S. Brodke, $M D$

\section{Midwest Orthopaedics at Rush}

Introduction: Reconstruction after vertebral resection for indications such as tumor, infection, or trauma typically involves posterior segmental fixation and anterior interbody support. However, factors such osteopenia, radiation, or the oncologic need for endplate resection make interbody device subsidence and failure a concern.

Methods: An idealized thoracolumbar spondylectomy model was constructed using Delrin vertebral bodies, posterior segmental fixation, and an anterior interbody cage. In-vivo thoracolumbar mechanical stress was simulated on a custom multi-axis spine simulator using 10 cycles of flexion-extension motion, $200 \mathrm{~N}$ of axial preload, and a $12 \mathrm{Nm}$ follower load. Rigid body position in space was measured using an optical motion-capture system. Cancellous subsidence was modeled using a $1 \mathrm{~cm}$-thick wafer of \#3 closed-cell Sawbones foam at one endplate. Ten foam specimens were tested in a control state consisting of posterior pedicle screw and rod fixation with a free and independent interbody cage. Ten additional foam specimens were tested with the Delrin interbody cage "connected" to the posterior rods using two additional pedicle screws placed into the cage. Foam indentation was quantified using a precision digital surface-mapping device.

Results: The control group exhibited significantly greater foam indentation after cycling, with a mean subsidence volume of 1906 $\mathrm{mm} 3(95 \%$ CI

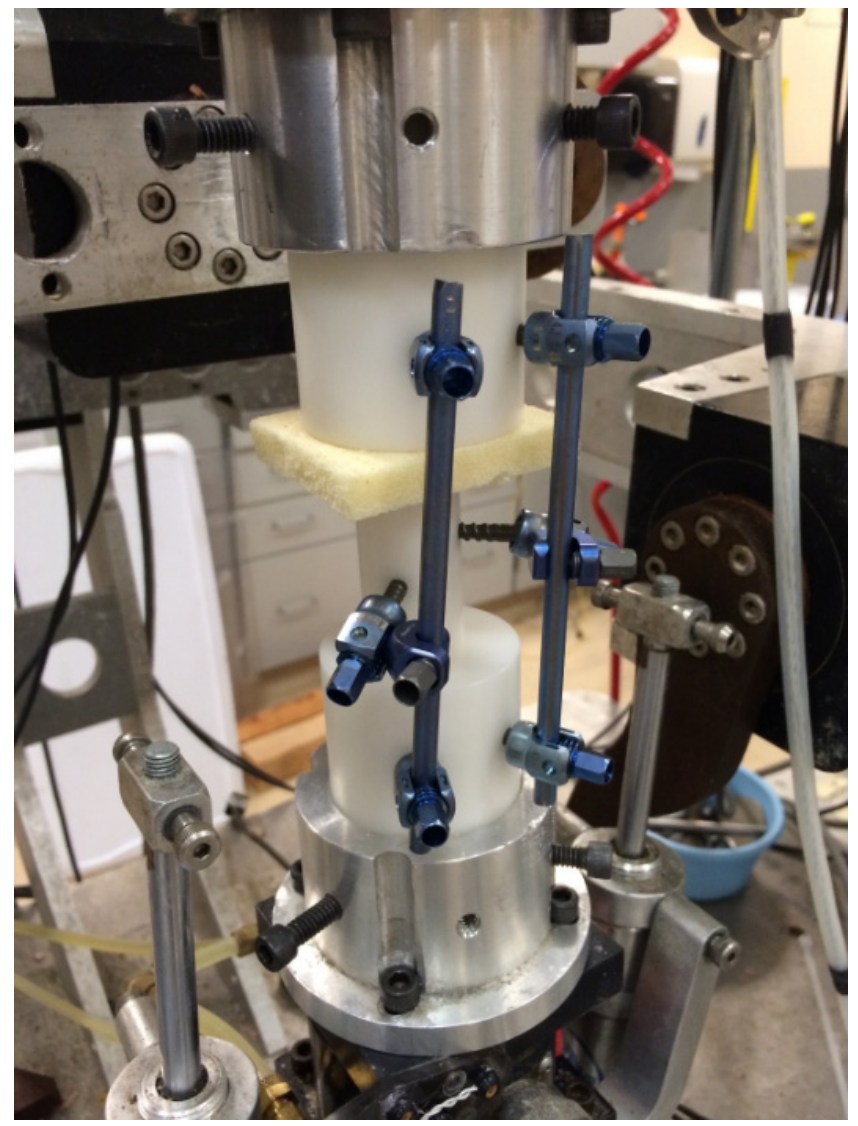

1810-2001 mm3) compared to the connected cage group subsidence volume of $977 \mathrm{~mm} 3$ (95\% CI 928-1026 mm3; p<0.001). Construct motion changed throughout cycling as the foam settled, but the steady state flexion- extension arc was significantly greater in the control group (8.4 degrees, 95\% CI 8.2-8.6 degrees) than in the connected cage group (6.1 degrees, 95\% CI 6.1-6.2 degrees; $\mathrm{p}<0.001)$. Correspondingly, construct stiffness was greater in the connected cage group (3.1 N/degree, 95\% CI 3.1-3.2 N/degree) compared with the control group (2.3 N/degree, $95 \%$ CI $2.2-2.4 \mathrm{~N} /$ degree; $\mathrm{p}<0.001)$.

Conclusion: In an idealized spondylectomy model, connecting the anterior column cage to the posterior instrumentation using pedicle screws results in a construct which is nearly $40 \%$ stiffer and exhibits $50 \%$ less cancellous subsidence compared with a traditional unconnected cage.

Discussion:Connecting posterior instrumentation directly to the anterior vertebral body replacement device following spondylectomy may help avoid implant-related failure.

Poster \# 26. Moving Beyond Radiographs; Functional Changes in the Sagittal Plane after Adolescent Idiopathic Scoliosis Realignment and Fusion

Ashish Patel, MD; Robert Pivec, MD; Bhaveen H. Kapadia, MD; Dante M. Leven, DO; Colin Cooper, MD; Arie Trouw, MD; Shikha Sheth, BA; Carl B. Paulino, MD

\section{SUNY Downstate Medical Center}

Introduction: The sagittal plane is recognized as the primary factor in successful clinical outcomes in patients with adult spinal deformities. Attention to restoring sagittal plane alignment and function is critically important during all spinal procedures. The 


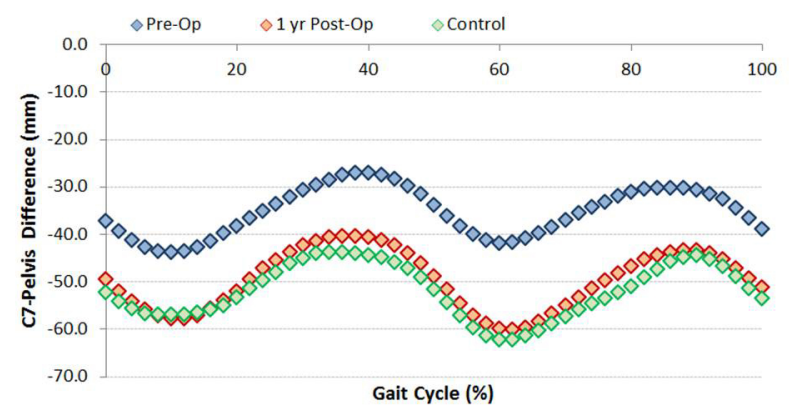

Figure 1. Plot of the $\mathrm{C} 7$ skin maker relative to the center of pelvis during one complete gait cycle for; control patients (green), pre-operative AIS patients (blue), and 1 year post-operative AIS patients (orange). Post-operative patients mirror control subjects, however increased sagittal plane motion is also observed.

objective of this study was to determine the dynamic properties in the sagittal plane after posterior spinal fusion for AIS.

Methods: Thirty consecutive AIS patients were prospectively enrolled into this gait analysis study. Analysis was performed in a 6-DOF motion analysis laboratory at a sampling frequency of 100 $\mathrm{Hz}$. Thirty-four reflective markers were placed on each patient who then underwent straight-line walking trials pre-operatively and at 1 and 2 yr follow-up. Sagittal marker data including C7-Pelvis offset an C7-Pelvis motion were calculated to determine the pre-post sagittal motion. Statistical analysis was performed with a Bonferronicorrected $p$ value $<0.05$ for significance.

Results: In control subjects, the C7 marker was a mean $51.9 \mathrm{~mm}$ posterior to the center of the pelvis (C7-Pelvis offset). C7-Pelvic motion was found to be $20 \mathrm{~mm}$ (from $43 \mathrm{~mm}-63 \mathrm{~mm}$ ) during the entire gait cycle. AIS patients ambulated significantly anterior to control subjects (C7-Pelvic offset $=35 \mathrm{~mm})$ with a significantly less C7-Pelvic motion of $15 \mathrm{~mm}$ (from $28 \mathrm{~mm}-43 \mathrm{~mm}$ ). Postoperatively, the C7-Pelvis shifted posteriorly to $49.7 \mathrm{~mm}(\mathrm{p}<0.01)$ and sagittal plane motion was found to increase $25 \%$ to $20 \mathrm{~mm}$ (from $40 \mathrm{~mm}$ - $60 \mathrm{~mm}$ ). In fact, cross-correlation of the post-operative sagittal C7-Pelvic offset during the whole gait cycle demonstrated a pattern that mirrored the control group (Rxy $=0.98 ; \mathrm{p}<0.001$; Figure 1$)$.

Conclusion: Posterior spinal fusion and realignment in AIS patients resulted in sagittal plane posture and C7-Pelvic offsets that significantly mirrored control subjects during straight line walking. Although favorable realignment may be obtained, long term studies are required to investigate the evolution of sagittal plane motion and function post-AIS fusion. With increased sagittal C7-Pelvic motion after long spinal arthrodesis, concern for the remaining un-fused segments and disc health transpire.

\section{Poster \# 27. Does Cage Subsidence Affect Clinical Outcomes After Transforaminal Lumbar Interbody Fusion?}

Scott Wagner, MD; Peter M. Formby, MD; Daniel G. Kang, MD; Melvin Helgeson, MD

\section{Walter Reed National Military Medical Center}

Introduction: Interbody cage subsidence after transforaminal lumbar interbody fusion (TLIF) is a known complication, and loss of height over time can lead to foraminal collapse and potentially recurrence of symptoms. The overall clinical relevance of radiographic subsidence remains unexplored. We compared the clinical outcomes of patients with and without radiographic evidence of interbody subsidence after TLIF.

Methods: We performed a retrospective review of a single academic institution with multiple spine surgeons over a ten year period. We included all patients over age 50 following TLIF with immediate post-operative and follow-up computed tomography
(CT) scans available for radiographic quantification of interbody cage subsidence. Medical records were reviewed for patient demographics, presenting complaints/symptoms, surgical data, persistence/recurrence of symptoms postoperatively, complications and occurrence of revision/additional surgery.

Results: We included 128 patients. Average follow up was 27.2 months and overall fusion rate was $56 \%$. Fifty-five (43\%) had evidence of interbody cage subsidence at most recent follow up, while the remaining $73(57 \%)$ patients had no implant subsidence on radiographs. There was no difference found in sex distribution, age (60.6 years vs 58.1 years, $\mathrm{p}=0.115)$, body mass index (BMI; 28.5 vs $29.2, \mathrm{p}=0.449)$ or lumbar vertebral body Hounsfield units $(149.8 \mathrm{HU}$ vs $160.8 \mathrm{HU}, \mathrm{p}=0.195)$ between subsidence and no-subsidence groups, respectively. Twenty-five (45\%) subsidence patients had persistence or recurrence of preoperative symptoms after surgery, compared to $38 \%$ of those without subsidence $(\mathrm{p}>0.05)$. There was a trend toward an increased revision rate in the subsidence group (16.4\%) compared to no-subsidence group $(8.2 \%, \mathrm{p}=0.08)$.

Discussion: Our findings suggest that interbody cage subsidence may not have an effect on persistence or recurrence of preoperative symptoms after TLIF. However, we found a trend toward an increased rate of revision surgery for subsidence, but the clinical consequences remain unclear.

Conclusion: There were no differences noted for post-operative recurrence or persistence of pain after TLIF in patients with subsidence of the cage. However, despite not reaching statistical significance, the revision rate in the subsidence group was twice that of the normal group. We hypothesize that the decision to revise may often be based on radiographic evidence of failure, rather than overall clinical outcomes.

\section{Poster \# 28. Validation of the Lumbosacral Injury Classification System (LSICS)}

Scott Wagner, MD; Gregory Van Blarcum, MD; Peter M. Formby, MD; Daniel G. Kang, MD; Ronald A. Lehman Jr., MD

\section{Walter Reed National Military Medical Center}

Introduction: There is currently no universally accepted classification or scoring system for lumbosacral dissociation (LSD) injuries. A new classification system called the lumbosacral injury classification system (LSICS) has been proposed based on three injury characteristics: injury morphology, neurologic status and posterior ligamentous complex (PLC) integrity. This new classification system for LSD injuries has not yet been validated in a systematic and scientific manner. Therefore, we set out to begin pre-clinical validation of the LSICS, and to assess for intra- and inter-observer reliability.

Methods: Four orthopedic surgeons performed separate reviews of nineteen LSD injury case examples, including injury mechanism, description of neurologic examination, and pertinent plain radiography and computed tomography (CT) scans. A composite LSICS injury severity score was calculated for each case. The reviews were performed in two rounds one week apart. Statistical analysis was performed to determine intra- and inter-observer reliability for the LSICS.

Results: After two rounds of scoring, we found inter-observer reliability values of $0.81,0.93$ and 0.5 for fracture morphology, neurologic status and posterior ligamentous integrity, respectively. Inter-observer reliability for the overall calculated severity score was 0.86 . Intra-observer reliability values were $0.76,0.94$ and 0.65 for all variables, respectively. Intra-observer reliability for the overall severity score was 0.87 .

Discussion: Our results suggest that LSICS is a reliable classification scheme for lumbosacral dissociation injuries, with high reproducibility within and between physicians. Integrity of the posterior ligamentous complex is the most difficult to assess 
radiographically, as evidenced by the lower reliability scores for that factor. We believe that with further analysis and previously described clinical modifiers, LSICS is predictive of injury severity and provides guidance for management of these complex injuries.

Conclusion: The results of this study have exemplified the high degree of inter-observer and intra-observer reliability with LSICS. Though lumbosacral dissociation injuries are rare, institutional implementation on LSICS will increase the reliability and effectiveness of this classification scheme, allowing the transfer of meaningful information between physicians. However, further validation using prospective clinical analysis must be performed.

Poster \# 29. A Meta-analysis On The Treatment Of Thoracolumbar Fractures: Open Versus Percutaneous Fixation

Steven McAnany, MD; Samuel Overley, MD; Jun Kim, MD; Evan Baird, MD; Sheeraz Qureshi, MD, MBA; Paul A. Anderson, MD

Mount Sinai Medical System

Study design: Systematic literature review and meta-analysis of English language studies.

Objective: This study is evaluating differences in outcomes variables between percutaneous and open pedsicle crews for traumatic thoracolumbar fractures.

Background information: Thoracolumbar fractures have historically been treated with an open posterior approach. Advances in minimally invasive spine surgery (MISS) have given rise to treatment alternatives in the field of spine trauma. There exists, hoever, no consensus in the literature on the optimal treatment modality.

Methods: A systematic review of Pubmed, Cochrane, and Embase was performed. The variables of interest included post-operative VAS pain score, kyphosis angle, and vertebral body height, as well as intraoperative blood loss and operative time. The pooled results were performed by calculating the effect size based on the standardized difference in means. Studies were weighted by the inverse of the variance, which included both within and between study error. Confidence intervals (CI) were reported at $95 \%$. Heterogeneity was assessed using the $\mathrm{Q}$ statistic and $\mathrm{I} 2$.

Results: After two-reviewer assessment, thirty-eight studies were eliminated. Six studies were found to meet inclusion criteria and were included in the meta-analysis. The quality index score of our nine studies ranged from fourteen to eighteen. We calculated anaverage score of 16.6 and a standard deviation of 1.5. We defined a higher quality study as $16-17$, a moderate quality study as $13-15$, and a poorer quality study as 8-12. There were five higher quality studies and one moderate quality study.

The combined effect size was found to be in favor of percutaneous fixation for blood loss and operative time $(\mathrm{p}<0.05)$; however, there were no differences in VBH, kyphosis angle, or VAS scores between open and percutaneous fixation. All of the studies demonstrated relative homogeneity, with $\mathrm{I} 2<25$.

Conclusions: Patients with thoracolumbar fractures can be effectively managed with percutaneous or open pedicle screw placement. There are no differences in VBH, kyphosis angle, or VAS between the two groups. Blood loss and operative time were decreased in the percutaneous group, which may represent a potential benefit, particularly in the polytraumatized patient. All variables in this study demonstrated near-perfect homogeneity, and the effect is likely close to the true effect.

Poster \# 30. Adult Spinal Deformity: National Trends in the Presentation, Treatment, and Peri-operative Outcomes over 10 Years

Cyrus Jalai, B.A.; Nancy J. Worley, MS; Shaleen Vira, MD; Peter G. Passias, $M D$

\section{NYU Hospital for Joint Diseases}

Introduction: This study investigates postsurgical outcomes for the surgical management of adult spinal deformity (ASD) and analyzes these trends on a nationwide scale using data provided by the Nationwide Inpatient Sample (NIS) from 2003-2010.

Methods: The Nationwide Inpatient Sample (NIS) database was retrospectively queried for adult spinal deformity patients above age 25 undergoing either an anterior, posterior, or combined surgical approach between 2003-2010. Patients with fractures, nine or more levels fused, or any cancer were excluded. The following data were collected: patient demographics, hospital system-related data, and total procedure-related complications. Yearly trends were analyzed using univariate analysis and linear regression modeling.

Results: 10,966 cases were identified. Yearly trend analysis shows that the number of anterior, posterior, and combined approaches did not significantly increase in frequency over the considered interval $(p>0.05)$. Total charges for anterior, posterior, and combined surgical approaches all increased significantly $(38.87 \%, 97.13 \%$, $54.86 \%$, respectively, $\mathrm{p}<0.05$ ). Total hospital length of stay did not significantly change for any of the considered surgical approaches over the time interval. Lastly, yearly trend analysis revealed that overall morbidity for all procedures increased by $51.72 \%(\mathrm{p}<0.001)$.

Discussion: For ASD surgery from 2003-2010, length of stay of has not changed, complications have increased, and surgical costs for all approaches have been rising regardless of surgical approach. The relative proportion of cases that utilized each surgical approach did not significantly differ over this time period.

Conclusion: In light of the current healthcare environment, novel strategies are required to develop innovative methods to reduce length of stay and reduce surgical costs.

Poster \#31. Resolution Of Lumbar Radicular Symptoms Following Standalone Anterior Or Lateral Interbody Fusion.

Adam C. Lipson, MD; David Poulad, MD

\section{IGEA Brain \& Spine}

Introduction: Lumbar radiculopathy is traditionally treated via direct decompression via posterior laminectomy and/or foraminotomy procedures. Recent publications on interbody fusions performed via the lateral transpsoas retroperitoneal approach (LLIF) indicate that there is an indirect decompression of the nerve roots which contributes to improvements in preoperative claudication and radiculopathy. There is scarce literature available regarding the outcomes for radiculopathy following anterior lumbar interbody fusion (ALIF).

Methods: We reviewed a total of 41 patients (mean age 52.8) who had radicular and axial back pain symptoms who had undergone initial 1- or 2-level stand-alone ALIF or LLIF without posterior decompression between 2009-2014. Indications for fusion included instability, grade 1 spondylolisthesis, stenosis, degenerative disc disease adjacent to a prior fusion, with or without prior surgery. All patients were evaluated by outcome analysis scales (VAS scores, Oswestry disability index - ODI) and radiographic follow-up. Segmental height, lordosis, listhesis, foraminal height and area were recorded. Follow-up ranged 3-42 months.

Results: 11 patients underwent LLIF, 30 patients underwent ALIF. 3 of the LLIF patients (27\%), and 4 of the ALIF patients $(13 \%)$ did not have any improvement in radicular pain relief, and underwent a staged posterior laminectomy and pedicle screw instrumentation within 1-7 weeks after the initial surgery. Of the remaining 34 patients in the stand-alone group there was an improvement in the back pain VAS from 7.6 to 2.7 in the LLIF group, 8.2 to 3.5 in the ALIF group. There was an improvement in the leg pain VAS from 7.2 to 1.7 in the LLIF group, and from 7.3 to 2.3 in the ALIF 
group. There were marked improvements in ODI for both standalone groups.

We are presently working on radiographic measurements, including subsidence rates. Arthrodesis was achieved in 11/11 LLIF patients and 29/30 ALIF patients.

Discussion: Stand-alone ALIF and LLIF procedures are effective at resolving radicular pain symptoms. Mechanisms of indirect decompression increasing the foraminal height and area through distraction with an interbody graft, tensing the spinal ligaments, and immobilizing the diseased spinal segment, The presence of stenosis may not necessitate a 360 degree approach to achieving a full direct decompression.

\section{Poster \#32. Quantification of Multifidus Atrophy and Fatty} Infiltration Following A Minimally Invasive Microdiscectomy

Ehsan Tabaraee, MD; Junyoung Ahn, BS; Daniel D. Bohl, MPH; Frank Phillips, MD; Kern Singh, MD

\section{Rush University Medical Center}

Introduction: The multifidus muscle is an important dynamic stabilizer of the spine. Degeneration, edema, and atrophy of the multifidus have been demonstrated in patients undergoing traditional open posterior spine surgery. The objective of this study is to quantify the extent and distribution of multifidus muscle atrophy and fatty infiltration following a 1- or 2-level unilateral, minimally invasive (MIS) laminectomy with discectomy.

Methods: Magnetic resonance imaging (MRI) of 31 patients who underwent a 1- or 2-level laminectomy with micro discectomy were reviewed. Total and lean cross-sectional area (CSA, mm2), T1 signal intensity ratio between multifidus and iliopsoas muscles, and lean to total CSA ratio were measured. Pre- and post-operative measurements were compared within each patient utilizing paired sample t-tests.

Results: 31 patients (14 female) were analyzed with a mean age of $50.6 \pm 14.3$ years. 23 patients underwent a 1-level decompression ( $n=8,2$-level decompression). Post-operative MRI was obtained on average $207.1 \pm 240.5$ days following index surgery. On the ipsilateral side, significant decreases were observed in total CSA at the index level $(4.2 \%$ decrease, $\mathrm{p}<0.05)$, and lean CSA at the superior pedicle level $(8.5 \%$ decrease, $\mathrm{p}<0.05)$, index level $(9.1 \%$ decrease, $\mathrm{p}<0.001)$, and inferior pedicle level $(11.4 \%, \mathrm{p}=<0.01$; Table 1$)$. On the contralateral side, significance decreases were demonstrated at

Table 1. MRI-Documented Changes in Multifidus Muscle Following 1- and 2-Level Primary Lumbar Decompression

\begin{tabular}{|c|c|c|c|c|c|c|c|c|}
\hline \multirow[b]{3}{*}{ Level } & \\
\hline & \multicolumn{4}{|c|}{ Multifidus Total Cross Sectional Area (mm2) } & \multirow{2}{*}{\multicolumn{4}{|c|}{ Multifidus Lean Cross Sectional Area (mm2) }} \\
\hline & Pre-operative & Post-operative & $\%$ Change & $p$-value & Pre-operative & & & p-value \\
\hline Supra Disc & $746.1 \pm 232.5$ & $770.8 \pm 252.7$ & $+3.7 \%$ & 0.17 & $543.4 \pm 204.4$ & $534.4 \pm 219.8$ & $-0.8 \%$ & 0.59 \\
\hline Supra Pedicle & $886.2 \pm 309.6$ & $894.4 \pm 339.8$ & $+1.5 \%$ & 0.80 & $665.8 \pm 273.5$ & $610.3 \pm 262.8$ & $-8.5 \%$ & $<0.05$ \\
\hline & $1040.6 \pm 23$ & $1000.6 \pm 23$ & $-4.2 \%$ & $<0.05$ & & & $-9.1 \%$ & $<0.001$ \\
\hline fra Pedicle & $1140.3 \pm 283.3$ & $1094.6 \pm 267.5$ & $-2.8 \%$ & 0.16 & $725.0 \pm 216.2$ & $632.3 \pm 202.2$ & $-11.4 \%$ & $<0.01$ \\
\hline \multirow[t]{3}{*}{ Infra Disc $\uparrow$} & $1020.5 \pm 297.9$ & $1098.5 \pm 252.6$ & $+3.2 \%$ & 0.17 & $653.2 \pm 242.1$ & & & 0.053 \\
\hline & \multicolumn{8}{|c|}{ Contralateral } \\
\hline & \multicolumn{4}{|c|}{ Multifidus Total Cross Sectional Area (mm2) } & \multirow{2}{*}{\multicolumn{4}{|c|}{$\begin{array}{l}\text { Multifidus Lean Cross Sectional Area (mm2) } \\
\text { Pre-operative Post-operative \% Change p-value }\end{array}$}} \\
\hline Level & Pre-operative & Post-operative & $\%$ Change & p-value & & & & \\
\hline Supra Di & & & & 0.53 & & & & 0.57 \\
\hline Supra & & & & & & & & 0.20 \\
\hline & 4 & & & & & & & $<0.05$ \\
\hline a Pedicle & & & $+3.6 \%$ & 0.26 & & & $-1.5 \%$ & 0.28 \\
\hline & & & +2 & 0.81 & 690. & & -2 & 0.23 \\
\hline & \multicolumn{8}{|c|}{ Multifidus Psoas T1 Sjonal Intensity Ratio } \\
\hline & \multicolumn{4}{|c|}{ Multifidus Psoas T1 Signal Intensity Ratio } & \multirow{2}{*}{\multicolumn{4}{|c|}{ Lean CSA to Total CSA Ratio }} \\
\hline Level & Pre-op & & \% Change & p-value & & & & \\
\hline Supra Disc & & & +17 & 0.67 & & & & $<0.05$ \\
\hline & & & & & & & & $<0$. \\
\hline & & & & & & & & $<0.01$ \\
\hline & & & & & & & & 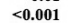 \\
\hline Infra $\mathrm{Di}$ & & & & 0.09 & & & $-14.6 \%$ & $<0.001$ \\
\hline & \multicolumn{8}{|c|}{ Contralateral } \\
\hline & & \\
\hline Level & Pre-ope & $\mathrm{e}$ & $\% \mathrm{Ch}$ & p-value & Pre-o & & & \\
\hline & & & & & & & & \\
\hline & & & & & & & & 0.23 \\
\hline & & & & & & & & 0.52 \\
\hline & & & +19 & & & & $-2.6 \%$ & \\
\hline & & & & 0.61 & & $0.61 \pm 0.14$ & $-6.6 \%$ & $<0$. \\
\hline
\end{tabular}

† Patients whose operative levels included S1 were excluded from measurement at the inferior disc level. the index level in total (2.7\% decrease, $\mathrm{p}<0.05)$ and lean CSA $(4.2 \%$ decrease, $\mathrm{p}<0.05)$. The $\mathrm{T} 1$ signal intensity ratios were increased following surgery at all levels, but no statistically significant differences were demonstrated. The lean/total CSA ratio was significantly decreased at all ipsilateral levels and at the contralateral inferior disc level.

Discussion: Findings of this analysis suggest 1- and 2-level MIS decompression and microdiscectomy results in minimal fatty degeneration of the multifidus muscle. The lean/total CSA ratio demonstrated only very small decreases at all ipsilateral levels and at the contralateral inferior disc level. Overall, approximately $90 \%$ of the lean muscle mass is maintained while chronic changes are confined to the area between the neighboring pedicles.

Conclusions: The integrity of the multifidus muscle appears to be radiographically preserved following an MIS lumbar decompression.

\section{Poster \# 33. The Impact of Socioeconomic Status on the Utilization of Spinal Imaging}

Adeeb Derakhshan, BS; Jacob A. Miller, BS; Daniel Lubelski, BA; Amy Nowacki, PhD; Ed C. Benzel, MD; Thomas Mroz, MD; Michael P. Steinmetz, MD

\section{Cleveland Clinic}

Introduction: One of the primary goals of the Patient Protection and Affordable Care Act is to mitigate disparities in access to healthcare among people of different socioeconomic (SES) classes. Differences in SES can manifest themselves through a variety of manners, including unequal access to medical imaging. The objective of this study was to assess the effect of socioeconomic status on the frequency with which imaging studies of the lumbar spine are ordered and completed.

Methods: Patients that were diagnosed with lumbar radiculopathy and/or myelopathy and had at least 1 subsequent lumbar MRI, CT, or X-ray ordered were identified. Demographic information and the number of ordered and completed imaging studies were among the data collected. Patients' zip codes served as proxies for socioeconomic status.

Results: A total of 24105 patients were stratified into four quartiles based on median income. Regression analyses demonstrated that patients in the lowest socioeconomic status quartile had higher rates of MRI, CT, and X-ray ( $\mathrm{p}<0.0001$ for all) imaging ordered. Uninsured patients were less likely to have imaging studies ordered ( $\mathrm{p}<0.0001$ for all modalities) and completed $(\mathrm{p}<0.0001$ for MRI and $\mathrm{X}$-ray). Physicians were less likely to order imaging studies for female ( $p=0.0006$ for MRI, $p=0.05$ for CT, and $p<0.0001$ for $\mathrm{X}$-ray) and older ( $\mathrm{p}<0.0001$ for MRI, $\mathrm{p}=0.04$ for X-ray) patients. Race and physician specialty were also independent predictors of the measured outcomes.

Discussion: Patients with lower SES may have more imaging ordered based on the higher disease prevalence present in this group. If a physician is aware of an uninsured patient's status, he/ she may refrain from ordering imaging or substitute a cheaper alternative in order to avoid out-of-pocket costs.

Conclusion: Disparities in image utilization based on characteristics such as patient socioeconomic and insurance status highlight a critical gap in access to healthcare. Physicians should work to mitigate the influence of such factors when deciding whether to order imaging studies, especially in light of the impending shift in healthy policy in the United States. 
Poster \#34. De-Rotational Maneuvers during Surgical Realignment for Adolescent Idiopathic Scoliosis. Are we Doing Enough?

Ashish Patel, MD; Robert Pivec, MD; Bhaveen H. Kapadia, MD; Dante M. Leven, DO; Colin Cooper, MD; Arie Trouw, MD; Shikha Sheth, BS; David Frumberg, MD; Carl B. Paulino, MD

\section{SUNY Downstate Medical Center}

Introduction: Intraoperative de-rotational maneuvers using pedicle screw fixation attempts to restore axial plane alignment. Previous studies have found severe AIS subjects to have an abnormal 'in-phase' gait in the axial plane compared to control subjects. The objective of this study was to evaluate axial plane motion in AIS patients' pre and post operatively and compare these findings with asymptomatic controls.

Methods: Thirty consecutive AIS patients were prospectively enrolled into this gait analysis study. Analysis was performed in a 6-DOF motion analysis laboratory at a sampling frequency of $100 \mathrm{~Hz}$. Thirty-four reflective markers were placed on each patient who then underwent straight-line walking trials pre-operatively and at 1 and $2 \mathrm{yr}$ follow-up. Comparisons of axial plane pelvic and thoracic motion were made between pre, post and control groups. Statistical analysis was performed with a Bonferroni corrected $\mathrm{p}$ value $<0.05$ for significance.

Results: As previously reported, pre-op AIS patients demonstrated an abnormal 'in-phase' pelvic and thoracic rotation (fig.1-a). Pre-op patients had restricted motion to the right and no counter rotation throughout the gait cycle. Control subjects demonstrated a normal 'anti phase' rotation with counter-rotation at $24 \%$ and $78 \%$ of the gait cycle (fig.1-c). Post-operative AIS patients had notable improvements in pelvic and thoracic rotation with counter-rotation occurring at $38 \%$ and $65 \%$ of the gait cycle and an increase in maximal thoracic rotation to the right from 0.01 (pre-op) to $1.60^{\circ}$ (post-op; $\mathrm{p}=0.034$ ).

Conclusion: AIS patients have restricted motion in the axial plane and demonstrate an abnormal 'in-phase' gait pattern. Although improvements were noted in post-operative patients, no patient demonstrated axial plane gait parameters similar to normal controls. Greater emphasis could be made to axial plane correction during AIS re-alignment procedures; restoration of normal axial plane. Intraoperative de-rotational maneuvers result in a more physiologic 'anti-phase' gait pattern post-operatively.

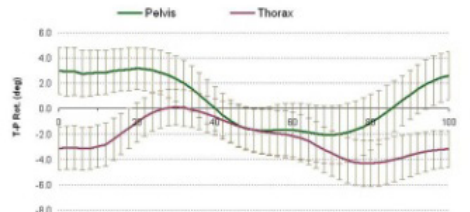

(A) and oresente

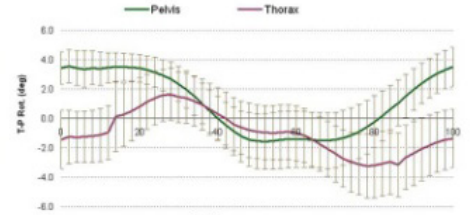

(B) $\arcsin (x)$

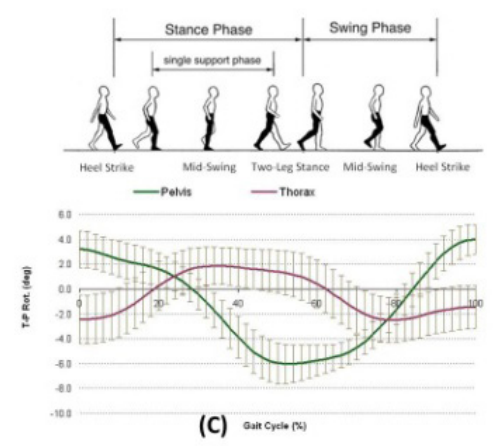
Figure 1a-c. Axial rotation of the pelvis and thorax for one gait cycle in pre-operative AIS (A), post-
operative AIS (B), and controls (C). Note thoracic and pelvic curves crossing at 2 points (anti-phase gait) operative AIS (B), and controls (C). Note thoracic and pelvic curves crossing at 2 points (anti-phase gait)
in controls and no crossing (in-phase gait) in pre-op AIS patients. Post Op AIS patients demonstrate a trend toward normal axial plane motion with counter rotation observed twice in their gait cycle.
Poster \#35. Adolescent Idiopathic Scoliosis Curves Cause Restricted Motion and Dis-Coordination of Axial Plane Motion

Dante M. Leven, DO; Ashish Patel, MD; Robert Pivec, MD; Bhaveen H. Kapadia, MD; Colin Cooper, MD; Arie Trouw, MD; Nathan A. Cornish, BS; Shikha Sheth, BS; Carl B. Paulino, MD

\section{SUNY Downstate Medical Center}

Introduction: It is well understood that Adolescent Idiopathic Scoliosis (AIS) is a 3-dimensional deformity. AIS results in structural and compensatory curves in the coronal plane, flattening of curves in the sagittal plane, and marked vertebral rotation toward the curve convexity in the axial plane. However, it is not well understood how spinal and vertebral modifications effect function. The objective of this investigation was to evaluate functional differences between a control group and patients with severe AIS, specifically in the axial plane.

Methods: Seventy two AIS patients, mean 14.5 years $(4 \mathrm{M}, 11 \mathrm{~F})$, with a mean Cobb angle of $57^{\circ}$ were prospectively enrolled into this gait analysis project. Analysis was performed in a 6-DOF motion analysis laboratory at a sampling frequency of $100 \mathrm{~Hz}$. Thirty-four reflective markers were placed on each patient who then underwent straight-line walking trials. Evaluations of pelvic and thoracic motion in the axial plane were made between the control group and the AIS group. All calculation for axial motion, patterns of gait, and phase were performed with custom algorithms. All statistical analysis was performed with a Bonferroni-corrected $\mathrm{p}$ value $<0.05$ for significance.

Results: Control subjects demonstrated a normal 'anti-phase' gait pattern. The pelvis and thorax were observed to rotate in opposite directions (anti-phase) during the gait cycle, reach a maximum pelvo-thoracic offset at heel strike, than rotate the opposite direction. The pelvis and thorax crossed each other twice during the gait cycle (' 0 ' point); once during right sided single limb stance ( $24 \%$ of gait cycle) and once during left sided single limb stance (78\% of gait cycle). Control subjects had a total pelvic rotation of $9.3^{\circ}$ and a combined pelvic-thoracic rotation of $199^{\circ}$. AIS patients demonstrated a mean $45 \%$ reduction in total pelvic rotation $\left(5.2^{\circ}\right)$ compared to controls $(\mathrm{p}<0.001$; Figure 1). AIS patients also demonstrated a mean $27 \%$ reduction in combined pelvic and thoracic rotation $\left(145^{\circ}\right)$ compared to controls $(\mathrm{p}<0.05)$. Compared to the control group, in AIS patients the pelvis and thorax did not cross at any point during the gait cycle, resulting in an abnormal 'in-phase' gait pattern.

Conclusions: Structural changes in patients with AIS are evaluated using radiographic methods although the functional impact of these structural changes was less understood. Our investigation demonstrates that severe AIS results in a restricted gait pattern in the axial plane with reduction in pelvo-thoracic motion.

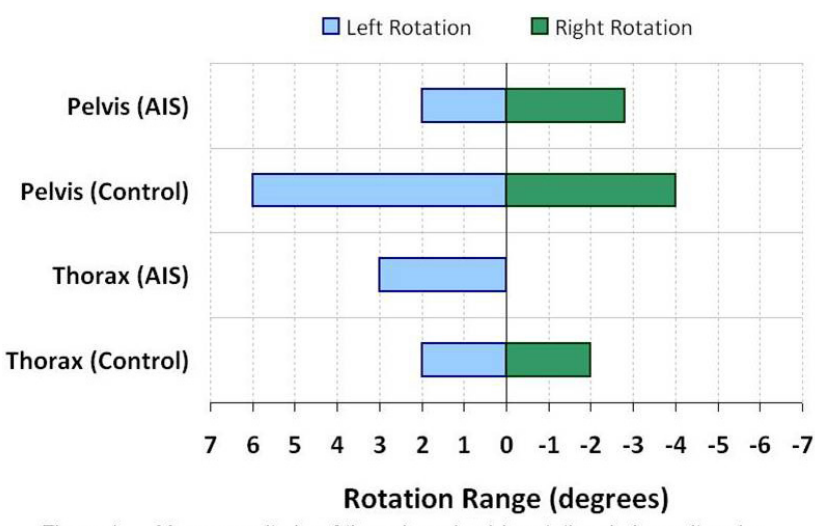

Figure 1. Mean magnitude of thoracic and pelvis rotation during gait cycle. 
Poster \#36. Moving Beyond Radiographs: Understanding the Functional Implications of Adolescent Idiopathic Scoliosis Correction in the Coronal Plane

Ashish Patel, MD; Robert Pivec, MD; Bhaveen H. Kapadia, MD; Nathan A. Cornish, BS; Dante M. Leven, DO; Colin Cooper, MD; Arie Trouw, MD; Shikha Sheth, BA; Carl B. Paulino, MD

\section{SUNY Downstate Medical Center}

Introduction: Coronal plane malalignment in AIS patents is the distinguishing feature that characterizes increasing AIS severity. With modern treatment techniques resulting in large radiographic coronal curve reductions, understanding the functional implications is the next step. The objective of this investigation was to investigate how intraoperative correction techniques alter coronal plane gait parameters.

Methods: Thirty consecutive AIS patients were prospectively enrolled into this gait analysis study. Analysis was performed in a 6-DOF motion analysis laboratory at a sampling frequency of $100 \mathrm{~Hz}$. Thirty-four reflective markers were placed on each patient who then underwent straight-line walking trials pre-operatively and at 1 and 2 yr follow-up. Comparisons of coronal motion were made between the study groups and a control group based on the position of $\mathrm{C} 7$, the pelvis, and the center of mass. Radiographic parameters including maximal Cobb angle and center sacral vertical line (CVSL) was also calculated. All calculations were performed with custom algorithms. All statistical analysis was performed with a Bonferroni-corrected $\mathrm{p}$ value $<0.05$ for significance.

Results: During gait analysis, control subjects demonstrated $3 \mathrm{~mm}$ of left-right sway in the coronal plane. Pre-operatively, AIS patients demonstrated $5 \mathrm{~mm}$ of left-right sway, post-operatively this reduced to $3 \mathrm{~mm}$, similar to control subjects. Statistical analysis demonstrated that there was a significant difference observed in the C7-Pelvis offset in pre-op AIS patients compared to both post-op AIS patients and control patients. There was no significant difference between C7-pelvis offset when comparing post-operative AIS patients to control patients. However, assessment of gait patterns demonstrated weak correlation $(\mathrm{r} 2=0.256, \mathrm{p}=0.01)$ between post-AIS patients and control subjects.

Conclusions: Coronal plane malalignment in AIS patents is the distinguishing feature that characterizes increasing AIS severity. Our investigation demonstrates that radiographic correction of Cobb angle and CVSL in AIS subjects results in post-operative gait motion similar to control subjects. It seems the balance center is restored and that left-right sway motion is reduced which is known to reduced energy consumption and thus a more efficient gait. With modern treatment techniques resulting in large radiographic coronal curve reductions understanding the functional implications is the next step.

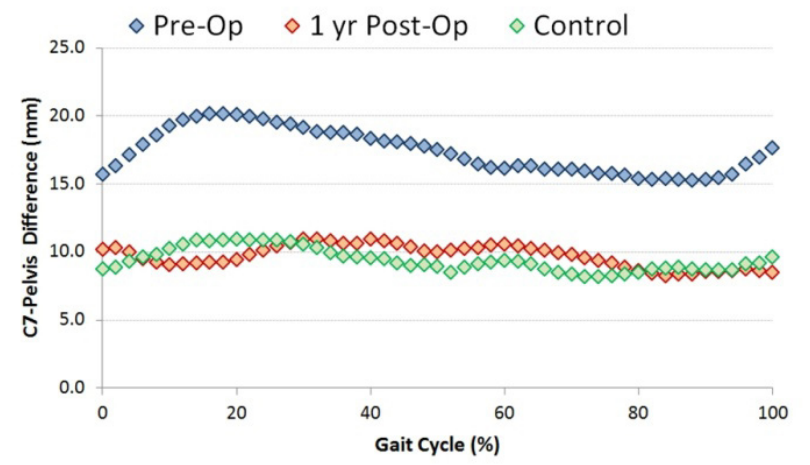

Poster \#37. Direct Current Stimulation for Spinal Fusion with a Piezoelectric Composite Material Interbody Implant: An Ovine Pilot Study

Elizabeth A. Friis, PhD; Sarah Galvis, BS; Paul M. Arnold, M.D.

\section{University of Kansas Medical Center}

Introduction: Only one direct current (DC) electrical stimulation set is clinically available, but it requires a second surgery site for battery pack implantation, has electrodes over the transverse processes, and may require re-operation for battery pack removal. An alternative is an interbody device incorporating a load-bearing piezoelectric composite implant material, which has been shown to generate the electrical stimulation needed for bone healing through the body's natural motion and loading. In this in vivo animal pilot study, we hypothesized that use of a piezoelectric composite lumbar spine interbody implant could reduce fusion time and enhance fusion quality.

Methods: Two of four prototype interbody implant devices were poled and made electrically active with circuitry designed to deliver a DC density of $5 \mu \mathrm{Amps} / \mathrm{cm} 2$ to the externally mounted electrode with implant loading. The two remaining identical control implants were not poled and did not have an external electrode or circuitry. Two skeletally mature female sheep underwent spinal fusion at L2-L3 and L4-L5 in accordance with an approved IACUC protocol. Both an active and control cage were placed in each animal in alternating locations. Post-implantation, the sheep ambulated normally, providing for cyclic loading across the spine. CTs were taken at six weeks and four months, at which time both sheep were sacrificed. The L2-L3 and L4-L5 spine segments from each sheep were loaded in displacement control to $5 \mathrm{Nm}$ in flexion/extension, bilateral bending, and bilateral axial torsion with displacement measurement by a position sensor. Overall range-of-motion (ROM) and extension zone (EZ) and neutral zone (NZ) stiffness and ROM were calculated.

Results: Six-week and four-month CTs showed substantially more new bone in the active piezoelectric implants compared to the controls. On average, the active implant fusion sites were stiffer than the controls in both NZ and EZ and had lower ROM, indicating more bone formation in the active implants compared to the controls.

Conclusions: The active piezoelectric composite implant fusion levels in both sheep showed more bony formation and better fusion compared to controls. Although statistical significance cannot be determined due to the low number of specimens, the results are promising.

Poster \# 38. The Stabilizing Impact of Fracture Screws and CrossConnectors in the Comparison of Short- and Long-Segment Posterior-Only Treatment of an Unstable Thoracolumbar Burst Fracture in a Cadaveric Model

Viktor Bartanusz, M.D., Ph.D.; Jonathan Andrew Harris, M.S.; Mark Moldavsky, M.S.; Yiwei Cai, B.S.; Omar Elkassabany, B.S.; Brandon Bucklen, Ph.D.

Globus Medical, Inc.

Introduction: Posterior-only techniques treating thoracolumbar burst fractures have increased in popularity due to minimal tissue disruption. Still, treatment remains a controversial. The aim of this study is to quantify the added stability of fracture screws and cross-connectors singularly, and in concert, for both short- and longfusion treatments in an unstable thoracolumbar burst fracture model.

Methods: Six fresh bovine spines were denuded and fixed at T11 and L5 using a 1:1 potting mixture. The unstable injury model was simulated through the sudden impulse of an axial load vertically released down a guided drop tower. Specimens were first incased 
into a first and second group as determined by the case number at which the procedural time distribution reached a plateau. Patient demographics, comorbidity burden (CCI), peri-operative variables, visual analogue scale (VAS) scores, post-operative complications, and re-operations were compared between the first 50 patients to the subsequent 178 patients. Statistical analysis was performed with Student's t-test for continuous and Chi-square test for categorical variables. Pearson's correlation coefficient was utilized to determine any association between case numbers and operative time and length of hospitalization. An alpha level of $<0.05$ denoted statistical significance.

Results: Patients in the initial cohort were older with a higher comorbidity burden $(\mathrm{p}<0.05)$. However, BMI and gender were similar between cohorts. Ethnicity and smoking status were significantly different between groups $(\mathrm{p}<0.001)$. The initial cohort incurred a greater procedural time $(52.2 \pm 22.2$ vs. $39.4 \pm 19.8 \mathrm{~min}, \mathrm{p}<0.001)$ and longer length of hospitalization $(22.1 \pm 25.4$ vs. $15.2 \pm 15.0$ hours, $\mathrm{p}<0.05)$ than the second cohort. Estimated blood loss, VAS scores (pre- and post-operative), complication rates, and re-operation rates were similar between groups. The primary cohort trended towards a higher re-operation rate with a slightly greater rate of recurrent disc herniations (not statistically significant).

Discussion: In this analysis, continued surgical experience was associated with a reduced operative time, shorter length of hospitalization, and similar blood loss following MIS lumbar decompression. Independent of surgical experience, all patients demonstrated similar improvements in clinical outcomes.

Conclusions: The findings of this study suggest that although surgical experience may improve peri-operative parameters (operative time, length of hospitalization), MIS lumbar decompression may initially be performed safely without prior experience.

Poster \#40. Single vs. Hypofractionated Focused Radiation Therapy: Effects on Vertebral Structure and Biomechanical Integrity

Christina Holmes, PhD; Benjamin D. Elder, MD/PhD; Jason Liauw, M.D.; Sheng-Fu L. Lo, MD; Ioan A. Lina, BS; Debebe Theodros, BS; Timothy F. Witham, MD

\section{Johns Hopkins University School of Medicine}

Introduction: Stereotactic radiosurgery has shown great promise in the treatment of metastatic lesions within the spine. However, recent clinical studies have indicated that the procedure may increase the risk of developing vertebral compression fractions, particularly in osteoporotic patients [Rose PS, et al., 2009. J Clinical Onc. 27(29):5075-79; Boehling NS, et al., 2012. J Neurosurg Spine. 16:379-386]. There is thus a critical need for research examining the effects of focused radiation on bone quality and mechanical properties. We have developed a rabbit model which enables the analysis of the effects of focused radiation on vertebral osteonecrosis, structure and biomechanical integrity. Using this model we are testing the hypothesis that fractionation of radiation dosing can reduce radiation osteonecrosis and preserve structural and biomechanical integrity of the spine.

Materials and Methods: The L5 vertebral body of New Zealand White (NZW) rabbits was treated, under computerized tomography (CT) guidance, with either a single $24 \mathrm{~Gy}$ dose of radiation or three fractionated doses over three consecutive days of 8 Gy radiation via the small animal radiation research platform (SARRP) [Wong J, et al, 2008. Int J Radiat Oncol Biol Phys. 71(5): 1591-1599]. Effects of radiation treatment on L2, L4, L5 and L6 vertebral osteonecrosis, structure and biomechanical integrity were evaluated 6 months post-irradiation via high-resolution CT-imaging, histology, and non-destructive biomechanical compression testing and compared to non-irradiated controls.

Results/Conclusions: Results to date suggest that a localized single dose of 24 Gy radiation leads to loss of vertebral bone volume and trabecular number and a subsequent increase in trabecular spacing. Hypofractionation of the radiation dose (3 x 8 Gy) similarly leads to reduced trabecular number and increased trabecular spacing, yet preserves normalized bone volume. Whether these changes in bone morphology translate into reduced mechanical integrity is currently under analysis. Future work will utilize this model to evaluate the efficacy of various therapies in the prevention of radiation induced osteonecrosis.

Disclaimer: The Journal of Neurosurgery Publishing Group (JNSPG) acknowledges that the preceding abstracts and poster sessions are published verbatim as submitted and did not go through either the JNSPG's peer-review or editing process. 